\title{
New topological and index theoretical methods to study the geometry of manifolds
}

\author{
Dissertation \\ zur Erlangung des mathematisch-naturwissenschaftlichen Doktorgrades \\ "Doctor rerum naturalium" \\ der Georg-August-Universität Göttingen \\ im Promotionsprogramm Mathematical Science \\ der Georg-August University School of Science (GAUSS) \\ vorgelegt von \\ Martin Nitsche \\ aus Kiel. \\ Göttingen, 2017
}




\section{Betreuungsausschuss}

Erstbetreuer: Prof. Dr. Thomas Schick,

Mathematisches Institut, Georg-August Universität Göttingen

Zweitbetreuer: Prof. Dr. Ralf Meyer, Mathematisches Institut, Georg-August Universität Göttingen

\section{Mitglieder der Prüfungskommission}

Referent: Prof. Dr. Thomas Schick, Mathematisches Institut, Georg-August Universität Göttingen

Korreferent: Prof. Dr. Ralf Meyer,

Mathematisches Institut, Georg-August Universität Göttingen

\section{Weitere Mitglieder der Prüfungskommission}

Prof. Dr. Dorothea Bahns,

Mathematisches Institut,

Georg-August Universität Göttingen

Prof. Dr. Viktor Pidstrygach,

Mathematisches Institut,

Georg-August Universität Göttingen

Prof. Dr. Karl-Henning Rehren,

Institut für Theoretische Physik,

Georg-August Universität Göttingen

Prof. Dr. Max Wardetzky,

Institut für Numerische und Angewandte Mathematik, Georg-August Universität Göttingen

Tag der mündlichen Prüfung: 06.02.2018 


\begin{abstract}
For a Spin manifold $M$ the Rosenberg index $\alpha([M])$ is an obstruction against positive scalar curvature metrics. When $M$ is non-Spin but $\operatorname{Spin}^{c}$, Bolotov and Dranishnikov suggested to apply the Rosenberg index to a suitable $S^{1}$ bundle $L \rightarrow M$. We study this approach, in particular for the case $\pi_{1}(L) \neq$ $\pi_{1}(M)$. We explain how the bundle construction can be turned into a nontrivial natural transformation of bordism groups $\Omega^{\text {Spin }^{c}} \rightarrow \Omega^{\text {Spin }}$. Then we show that $\alpha([L]) \in K O\left(C^{*}\left(\pi_{1}(L)\right)\right)$ always vanishes, but also give an example where $L$ nonetheless does not admit a positive scalar curvature metric.

The second part of the thesis concerns the relation of $\alpha([N])$ and $\alpha([M])$ for certain codimension-2 submanifolds $N \subset M$. Following a construction of Engel we extend the Thom map $K O_{*}(M) \rightarrow K O_{*-2}(N)$ to $K O_{*}\left(\mathbf{B} \pi_{1}(M)\right) \rightarrow$ $K O_{*-2}\left(\mathbf{B} \pi_{1}(N)\right)$, and then further to $K O_{*}^{\pi_{1}(M)}\left(\underline{\mathbf{E}} \pi_{1}(M)\right) \rightarrow K O_{*-2}^{\pi_{1}(N)}\left(\underline{\mathbf{E}} \pi_{1}(N)\right)$.
\end{abstract}




\section{Contents}

\begin{tabular}{lll}
\hline & Introduction & 2
\end{tabular}

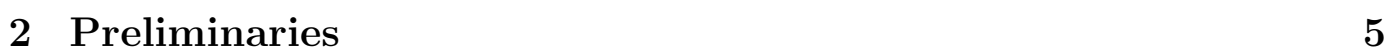

$2.1 \quad$ Positive scalar curvature metrics . . . . . . . . . . . . . . . 5

2.2 Spin-structures, Spin $^{c}$-structures and their obstructions . . . . . 7

2.3 Bordism and KO-homology $\ldots \ldots \ldots \ldots$

2.4 The index-theoretical obstruction . . . . . . . . . . . . . . . . 13

\begin{tabular}{|lll}
3 & The effect of changing the Spin structure & 17
\end{tabular}

4 The $S^{1}$-bundle construction 22

4.1 Motivation: Bolotov and Dranishnikov's article . . . . . . . . . 22

4.2 The $S^{1}$-bundle construction . . . . . . . . . . . . . . . . 24

4.3 The fundamental group of the $S^{1}$-bundle . . . . . . . . . . . . . 26

4.4 A natural transformation from $\Omega^{\text {Spin }^{c}}$ to $\Omega^{\text {Spin }} \ldots \ldots . . . . .32$

4.5 Rosenberg's example . . . . . . . . . . . . . . . . . . . . 36

4.6 The operator algebra side . . . . . . . . . . . . . . . . . . . . . . . . . . . 48

4.7 Vanishing of the index . . . . . . . . . . . . . . . . . . . . . . . . . . 41

4.8 Example for a non-psc circle bundle . . . . . . . . . . . . . . . . 46

$\begin{array}{lll}5 & \text { The codimension-2 transfer } & 48\end{array}$

5.1 Motivation . . . . . . . . . . . . . . . . . . . . . . . 48

5.2 Examples and restrictions on the fundamental groups . . . . . . 49

$5.3 \quad$ Extending the transfer map . . . . . . . . . . . . . . 52

$5.4 \quad$ Extending the transfer map further to $\mathbf{E} G$. . . . . . . . . . . . 60

\begin{tabular}{ll}
\hline Bibliography & 64
\end{tabular} 


\section{Introduction}

The notion of scalar curvature is perhaps the simplest way to measure how the local geometry of a Riemannian manifold differs from Euclidean space. For a long time there has been interest in the question which scalar curvature functions can be realized by a Riemannian metric on a given smooth closed manifold $M$. As it turns out, this problem mostly boils down to the question whether $M$ allows a Riemannian metric such that the scalar curvature is (strictly) positive everywhere.

One main tool in the study of this problem are index-theoretical obstructions against positive scalar curvature (psc) metrics. When the manifold $M$ is Spin, the Dirac operator gives rise to a fundamental class $[M]_{K O} \in K O(M)$ in real K-homology. If $M$ allows a psc metric, then the Rosenberg index of this class, $\alpha([M]) \in K O_{n}\left(C^{*}\left(\pi_{1}(M)\right)\right)$ is the zero element in the real K-theory of the group $C^{*}$-algebra of the fundamental group. This means that $\alpha([M])$ can be used as an obstruction against the existence of psc metrics on $M$.

There are also several geometrical constructions that can be used to study the psc question, such as fiber bundles, submanifolds and bordism. In this thesis we study how the index-theoretical obstruction combines with and relates to certain geometrical constructions in two different settings: In the first part, we apply the index obstruction to $S^{1}$-principal bundles over a $\operatorname{Spin}^{c}$ manifold $M$ and investigate if it can be used as an obstruction against psc metrics on $M$. In the second part, we consider certain codimension-2 submanifolds $N \subset M$ and try to relate the Rosenberg index of $M$ to that of the submanifold $N$.

We will begin by recapitulating in Section 2 the most essential concepts used in the following sections. This includes the notion of Spin- and Spin ${ }^{c}$ structures, bordism groups and the index-theoretical obstruction.

In a small detour, we calculate in Section 3 the effect that a change of the Spin-structure on $M$ has on the Rosenberg index $\alpha([M])$. This recovers the known fact that the vanishing of $\alpha([M])$ does not depend on the choice of the Spin-structure on $M$.

Theorem 1.1. Let $s_{1}, s_{2}$ be two Spin-structures on a closed connected smooth manifold $M$, related by the action of an element $x \in H^{1}\left(M ; \mathbb{Z}_{2}\right)$.

Then the Rosenberg indexes of the corresponding fundamental classes $[M]_{1}$, $[M]_{2}$ are related by the formula

$$
\alpha\left([M]_{2}\right)=\left(\Phi_{x}\right)_{*} \circ \alpha\left([M]_{1}\right),
$$

where $\Phi_{x}: C^{*}\left(\pi_{1}(M)\right) \rightarrow C^{*}\left(\pi_{1}(M)\right)$ is a $C^{*}$-automorphism that only depends on the class $x$.

In Section 4, the main part of this thesis, we investigate an approach that was suggested by Bolotov and Dranishnikov ([BD14]) in order to deal with a 
manifold $M$ that is not Spin but $\operatorname{Spin}^{c}$. In this setting the index obstruction cannot be applied directly, but it is possible to construct an $S^{1}$-principal bundle $L \rightarrow M$ such that $L$ is Spin. Then the obstruction can be applied to $L$ and a differential geometry argument shows that if $L$ is not psc, then neither is $M$.

In the case $\pi_{1}(L)=\pi_{1}(M)$ considered by Bolotov and Dranishnikov it turns out that the obstruction for $L$ always vanishes due to a bordism argument. When $\pi_{1}(L) \neq \pi_{1}(M)$, however, things are much less clear. We explain the $S^{1}$-bundle construction and the role of the fundamental group of the bundle, which is a group extension of $\pi_{1}(M)$. We also show how the choices made in the $S^{1}$-bundle construction can be made in a canonical way, giving rise to a natural transformation of bordism groups $\Omega_{*}^{\text {Spin }} \rightarrow \Omega_{*+1}^{\text {Spin }^{c}}$.

Theorem 1.2. For every odd number $n \in \mathbb{N}, n>1$, there is a non-trivial natural transformation $\Omega_{*}^{\text {Spin }^{c}}(\cdot) \rightarrow \Omega_{*+1}^{\text {Spin }}\left(\cdot \times \mathbf{B} \mathbb{Z}_{n}\right)$.

In the general case (with not necessarily canonical choices) we investigate the bordism group $\Omega^{\operatorname{Spin}}\left(\mathbf{B} \pi_{1}(L)\right)$ and the K-theory group $K O\left(C^{*}\left(\pi_{1}(L)\right)\right)$ and show that in many cases the absence of odd torsion in the latter groups forces the index obstruction to vanish. At the same time, however, a related example due to Rosenberg suggests that the obstruction might be non-zero in some cases. By using equivariant Spin-bordism groups we show that the obstruction does vanish in all cases. On the other hand, we give an example where the minimal hypersurface method of Schoen and Yau can be used to show that the $S^{1}$-bundle does not allow a psc metric.

Theorem 1.3. Let $M$ be a closed connected Spinc manifold such that $\widetilde{M}$ is not Spin and let $L \rightarrow M$ be an $S^{1}$-bundle that is Spin.

Then the Rosenberg index of $L$ inside $K O\left(C^{*}\left(\pi_{1}(L)\right)\right)$ always vanishes. At the same time there are examples where $L$ does not allow a metric of positive scalar curvature.

In Section 5 we consider the Rosenberg index of a codimension-2 submanifold $N \subset M$ with trivial normal bundle. Hanke, Pape and Schick showed ([HPS15]) that if the induced map $\pi_{1}(N) \rightarrow \pi_{1}(M)$ is injective and $\pi_{2}(N) \rightarrow$ $\pi_{2}(M)$ is surjective, then $\alpha([N]) \in K O_{n-2}\left(C^{*}\left(\pi_{1}(N)\right)\right)$ is an obstruction against psc metrics on $M$. The relation between $\alpha([M])$ and $\alpha([N])$ is unknown; ideally there might be a homomorphism $K O_{n}\left(C^{*}\left(\pi_{1}(M)\right)\right) \rightarrow K O_{n-2}\left(C^{*}\left(\pi_{1}(N)\right)\right)$ sending one to the other.

So far such a homomorphism has not been found. However, we can make a step in this direction by constructing on the topological side a transfer map $\operatorname{tr}_{M}: K O_{n}\left(\mathbf{B} \pi_{1}(M)\right) \rightarrow K O_{n-2}\left(\mathbf{B} \pi_{1}(N)\right)$ that sends the class $u_{M *}\left([M]_{K O}\right)$ to $u_{N *}\left([N]_{K O}\right)$, where $u_{M}$ and $u_{N}$ are classifying maps for the universal coverings. We explain the construction of this extension and discuss some examples where it can be applied. Then we show that the transfer map can be extended even further to $K O_{n}^{\pi_{1}(M)}\left(\underline{\mathbf{E}} \pi_{1}(M)\right) \rightarrow K O_{n-2}^{\pi_{1}(N)}\left(\underline{\mathbf{E}} \pi_{1}(N)\right)$. 
Theorem 1.4. Let $M$ be a closed connected Spin-manifold and $N$ a closed connected codimension-2 submanifold with trivialized normal bundle. Assume that $\pi_{1}(N) \rightarrow \pi_{1}(M)$ is injective and $\pi_{2}(N) \rightarrow \pi_{2}(M)$ is surjective.

Then there is, for any generalized multiplicative equivariant cohomology theory E with lf-restrictions, a map

$$
\operatorname{tr}_{\pi_{1}(M)}: E_{*}^{\pi_{1}(M)}\left(\underline{\mathbf{E}} \pi_{1}(M)\right) \rightarrow E_{*-2}^{\pi_{1}(N)}\left(\underline{\mathbf{E}} \pi_{1}(N)\right)
$$

such that the following diagram commutes:

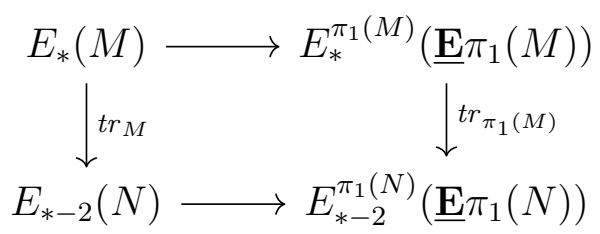

Moreover, the transfer $\operatorname{tr}_{\pi_{1}(M)}$ is natural for multiplicative transformations of equivariant cohomology theories with lf-restrictions.

\section{Acknowledgments}

I want to thank my advisor Thomas Schick for many fruitful discussions, for sharing his knowledge and for introducing me to the field of index theory in the first place.

I also thank my second advisor Ralf Meyer for the discussions during the annual meetings.

Funding acknowledgments: This thesis was partially supported by the German Academic Scholarship Foundation, and partially supported by the German Research Foundation (DFG) through the Research Training Group "Mathematical structures in modern quantum physics" at the University of Göttingen. 


\section{Preliminaries}

\subsection{Positive scalar curvature metrics}

When $M$ is a Riemannian manifold, the scalar curvature function $\kappa: M \rightarrow \mathbb{R}$ is defined to be the trace of the Ricci tensor. It is the simplest notion of curvature and measures how the volume of a small ball in $M$ differs from the volume of a ball of the same radius in Euclidean space:

$$
\frac{\operatorname{Vol}\left(B_{\epsilon}(x) \subset M\right)}{\operatorname{Vol}\left(B_{\epsilon}(0) \subset \mathbb{R}^{\operatorname{dim}(M)}\right)}=1-\frac{\epsilon^{2} \cdot \kappa(x)}{6 \cdot \operatorname{dim}(M)+2}+O\left(\epsilon^{4}\right) .
$$

In dimension 2 the scalar curvature is just twice the Gaussian curvature. In arbitrary dimension its value at a point $x \in M$ equals twice the sum of the sectional curvatures of all planes $e_{i} \wedge e_{j}, i<j$, where $\left\{e_{i}\right\}_{\leq n}$ is an orthonormal basis of $T_{x} M$.

It is natural to ask which scalar curvature functions can be realized by a Riemannian metric on a given manifold. This question is largely answered by the following Trichotomy Theorem due to Kazdan and Warner ([KW75a], [KW75b]):

Theorem 2.1.1. Let $M$ be a closed connected smooth manifold of dimension $\geq 3$. Then exactly one of the following statements is true:

1. All smooth functions $\mu: M \rightarrow \mathbb{R}$ can be realized as the scalar curvature of a Riemannian metric on $M$.

2. A smooth function $\mu: M \rightarrow \mathbb{R}$ can be realized as the scalar curvature of a Riemannian metric on $M$ iff it is either negative at some point or identically 0. In the latter case the Riemannian metric must be Ricci-flat, meaning its Ricci curvature vanishes everywhere.

3. A smooth function $\mu: M \rightarrow \mathbb{R}$ can be realized as the scalar curvature of a Riemannian metric on $M$ iff it is negative somewhere.

Setting aside the special case of Ricci-flat metrics, the crucial question remains whether a given manifold admits a metric of positive scalar curvature. In this case we say that $M$ is psc. We say that $M$ is not psc if it does not allow a positive scalar curvature metric.

Example 2.1.2. For $n \geq 2$ the usual metric on the sphere $S^{n}$, that comes from the standard embedding into $\mathbb{R}^{n+1}$, has positive scalar curvature.

Example 2.1.3. When $M$ is an arbitrary closed manifold, the product $M \times S^{2}$ allows a positive scalar curvature metric. Indeed, the scalar curvature of a product manifold is just the sum of the scalar curvatures of the individual 
manifolds. Since $M$ is closed, the scalar curvature of an arbitrary fixed metric on $M$ is bounded below. By scaling the metric of $S^{2}$, the curvature on $S^{2}$ can be made as large as needed such that the product metric on $M \times S^{2}$ has positive scalar curvature.

Example 2.1.4. In any dimension $n$ the torus $\mathbb{T}^{n}$ can be given a flat metric. But with the index-theoretical methods described below it can be shown that $\mathbb{T}^{n}$ does not allows a psc metric.

The main tool for showing that a given manifold does allow a psc metric is surgery. Gromov and Lawson, and independently Schoen and Yau, showed the following ([GL80], [SY79]):

Theorem 2.1.5. Let $N$ be a closed psc manifold, and let $M$ be obtained from $N$ by surgery of codimension $\geq 3$. Then $M$ allows a psc metric.

The idea here is that the codimension is high enough for the transversal sphere of the surgery to allow a psc metric. The difficult part is to find a suitable transition from the psc metric on the handlebody to the psc metric on $N$ near the points where the handle is attached to $N$.

What makes Theorem 2.1.5 particularly powerful is the fact that a bordism $W$ from $M_{1}$ to $M_{2}$ has a handle decomposition without handles of dimension $\leq 2$ if the inclusion $M_{1} \subset W$ is 2-connected ([Ran02], proof of 8.31). Reversing the handlebody decomposition, $M_{1}$ can then be obtained from $M_{2}$ by surgery in codimension $\geq 3$. Therefore, if $M_{2}$ is psc, then so is $M_{1}$.

This observation suggests that the psc question for a manifold $M$ can be decided by looking at the bordism classes $u_{*}\left([M]_{\text {Spin }}\right) \in \Omega^{\operatorname{Spin}}\left(\mathbf{B} \pi_{1}(M)\right)$ or $u_{*}\left([M]_{S O}\right) \in \Omega^{S O}\left(\mathbf{B} \pi_{1}(M)\right)$, where $u: M \rightarrow \mathbf{B} \pi_{1}(M)$ is the classifying map for the universal covering of $M$. (The reference map $u$ is used to avoid surgery in low (co)dimension.)

Gromov and Lawson showed ([GL80]):

Theorem 2.1.6. Let $M$ be a simply connected closed smooth manifold of dimension $\geq 5$ such that $\widetilde{M}$ is not Spin.

Then $M$ allows a psc metric.

Stolz and Jung showed ([RS01], 4.11):

Theorem 2.1.7. Let $M$ be a closed connected oriented manifold of dimension $\geq 5$ such that $\widetilde{M}$ is not Spin.

If the class $u_{*}([M]) \in H_{n}\left(\mathbf{B} \pi_{1}(M) ; \mathbb{Z}\right)$ can be represented by a psc manifold, then $M$ is psc.

To show that a manifold does not allow a psc metric, there are three main tools. Firstly, in dimension 4 the Seiberg-Witten invariant can be used. The second tool is the minimal hypersurface method due to Schoen and Yau [SY79], presented here in the formulation of Schick ([Sch98], 1.6): 
Theorem 2.1.8. Let $X$ be any topological space and let

$$
H_{m}^{+}(X)=\left\{f_{*}([M]) \in H_{m}(X ; \mathbb{Z}) \mid f: M \rightarrow X \text { and } M \text { is a psc manifold }\right\}
$$

For $3 \leq m \leq 7$ taking cap product with any $a \in H^{1}(X ; \mathbb{Z})$ maps $H_{m}^{+}(X)$ into $H_{m-1}^{+}(X)$.

Since $H_{2}^{+}(X)$ is just the image of the Hurewicz map $\pi_{2}(X) \rightarrow H_{2}(X ; \mathbb{Z})$, it can be computed easily. Theorem 2.1.8 can then be applied iteratively, for example to $f=\mathrm{id}: M \rightarrow M$, in the hope of leading the assumption that $M$ is psc into a contradiction.

The reason for the dimension restriction is that the proof uses Federer's regularity theorem [Fed70] to show that a codimension-1 immersed submanifold with (locally) minimal volume is in fact embedded. Lohkamp has announced a way to overcome these technical limitations [Loh] and very recently there is a preprint by Schoen and Yau where the result is proved without the dimension constraints [SY17].

Finally, if $M$ allows a Spin-structure on its tangent bundle, index theory provides an obstruction against the existence of a positive scalar curvature metric. We will explain this in more detail below.

\subsection{Spin-structures, Spin ${ }^{c}$-structures and their obstructions}

In this section we recapitulate some basic facts about Spin-structures and Spin $^{c}$-structures.

Definition 2.2.1. Let $\psi: G \rightarrow H$ be a homomorphism of topological groups and let $P_{H} \rightarrow X$ be a concrete (i.e., not up to bundle isomorphism) principal $H$-bundle over $X$.

Then a $G$-structure on $P_{H}$ is represented by a principal $G$-bundle $P_{G} \rightarrow X$ and a bundle map $\Phi: P_{G} \rightarrow P_{H}$ such that $\psi(g) . \Phi(q)=\Phi(g . q)$ for all $g \in G$, $q \in P_{G}$.

Two bundle maps $\Phi: P_{G} \rightarrow P_{H}$ and $\Phi^{\prime}: P_{G}^{\prime} \rightarrow P_{H}$ represent the same $G$-structure iff there exists a $G$-bundle isomorphism $\Psi: P_{G} \rightarrow P_{G}^{\prime}$ making the following diagram commutative:

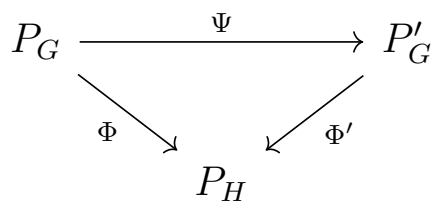


For example, an $n$-dimensional vector bundle $E \rightarrow X$ is orientable iff there exists an $S O(n)$-structure on the $O(n)$-bundle of orthonormal frames in $E$ (for any metric on $E$ ). Different orientations correspond to different $S O(n)$ structures.

We are interested in $\operatorname{Spin}(n)$ - and $\operatorname{Spin}^{c}(n)$-structures over a given $S O(n)$ or $O(n)$-bundle.

Definition 2.2.2. For $n \geq 3$ the topological group $\operatorname{Spin}(n)$ is defined to be the universal covering of $S O(n)$, which is a two-fold covering. For $n=2$, $\operatorname{Spin}(n)$ is the non-trivial two-fold covering, for $n=1$ it is the discrete group with two elements. $\mathbb{Z}_{2}$ acts on Spin via deck transformation.

$\operatorname{Spin}^{c}(n)$ is defined as the topological group

$$
\operatorname{Spin}^{c}(n)=\operatorname{Spin}(n) \times_{\mathbb{Z}_{2}} S^{1}=\left(\operatorname{Spin}(n) \times S_{1}\right) / \mathbb{Z}_{2},
$$

where $\mathbb{Z}_{2}$ acts on $S^{1}=U(1)$ by multiplication with \pm 1 .

There are canonical maps $\operatorname{Spin}(n) \rightarrow \operatorname{Spin}^{c}(n) \rightarrow S O(n)$ given by inclusion and projection.

Remark 2.2.3. When $P_{S O(n)} \rightarrow P_{O(n)}$ represents an $S O(n)$-structure on a given $O(n)$-bundle $P_{O(n)}$, and $P_{\operatorname{Spin}(n)} \rightarrow P_{S O(n)}$ represents a $\operatorname{Spin}(n)$-structure on $P_{S O(n)}$, then the concatenation $P_{\operatorname{Spin}(n)} \rightarrow P_{O(n)}$ represents a $\operatorname{Spin}(n)-$ structure on $P_{O(n)}$.

In the other direction, if $P_{\operatorname{Spin}(n)} \rightarrow P_{O(n)}$ represents a $\operatorname{Spin}(n)$-structure on $P_{O(n)}$, it induces an $S O(n)$-structure on $P_{O(n)}$, which is represented by the associated bundle $P_{\operatorname{Spin}(n)} \times{ }_{\operatorname{Spin}(n)} S O(n)$, and which only depends on the original $\operatorname{Spin}(n)$-structure.

Furthermore, if $P_{\operatorname{Spin}(n)} \rightarrow P_{O(n)}$ and $P_{S \sin (n)}^{\prime} \rightarrow P_{O(n)}$ give rise to the same $S O(n)$-structure, then the associated $S O(n)$-bundles are isomorphic over $P_{O(n)}$. In this particular case $(S O(n) \rightarrow O(n))$ the isomorphism is uniquely determined, making it possible to compare $P_{\operatorname{Spin}(n)} \rightarrow P_{\operatorname{Spin}(n)} \times_{\operatorname{Spin}(n)} S O(n)$ with $P_{\operatorname{Spin}(n)}^{\prime} \rightarrow P_{\operatorname{Spin}(n)}^{\prime} \times_{\operatorname{Spin}(n)} S O(n)$ as $\operatorname{Spin}(n)$-structures over a fixed $S O(n)$ bundle. In this sense one can say that picking a $\operatorname{Spin}(n)$-structure over an $O(n)$-bundle is the same as first picking an orientation and then picking a $\operatorname{Spin}(n)$-structure over any $S O(n)$-bundle representing this orientation.

This simplification does not work for the situation $\operatorname{Spin}(n) \rightarrow \operatorname{Spin}^{c}(n) \rightarrow$ $S O(n)$ because the bundle isomorphism between two representatives for the same $\operatorname{Spin}^{c}(n)$-structure on $P_{S O(n)}$ is not unique.

There is a second - homotopy-theoretic - definition of $G$-structures.

Definition 2.2.4. Let the classifying spaces $\mathbf{B} G$ and $\mathbf{B} H$ be represented by models such that the map $\mathbf{B} G \rightarrow \mathbf{B} H$ induced by the group homomorphism $\psi: G \rightarrow H$ is a fibration. And let $f: X \rightarrow \mathbf{B} H$ be a concrete topological map (i.e. not up to homotopy). 
Then a $G$-structure on $f$ is represented by a lift over $f$

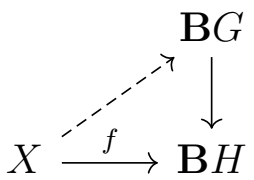

and two lifts represent the same $G$-structure iff they can be connected by a homotopy over $f$.

In the case that the (concrete) bundle $P_{H}$ is pulled back from $B H$ this definition is equivalent to the first one (see [Las63]).

The homotopy-theoretic definition is not only more general, it can also be used to answer the existence question for $G$-structures on a given $H$ bundle in a nice way. In particular, for $n \geq 3$ the maps $\operatorname{Spin}(n) \rightarrow S O(n)$, $\operatorname{Spin}^{c}(n) \rightarrow \operatorname{SO}(n)$ and $\operatorname{Spin}(n) \rightarrow \operatorname{Spin}^{c}(n)$ induce isomorphisms on all homotopy groups except for the fundamental group, where they induce either injections or surjections. It follows that the homotopy fibers of the induced maps on the classifying spaces are Eilenberg-MacLane spaces:

$$
\begin{aligned}
& K\left(\mathbb{Z}_{2}, 1\right) \rightarrow \mathbf{B} \operatorname{Spin}(n) \rightarrow \mathbf{B} S O(n) \\
& K(\mathbb{Z}, 2) \rightarrow \mathbf{B} \operatorname{Spin}^{c}(n) \rightarrow \mathbf{B} S O(n) \\
& K(\mathbb{Z}, 1) \rightarrow \mathbf{B} \operatorname{Spin}(n) \rightarrow \mathbf{B} \operatorname{Spin}^{c}(n)
\end{aligned}
$$

From obstruction theory (see [DK01]) it follows that the obstruction against existence of a $\operatorname{Spin}(n)$ - or $\operatorname{Spin}^{c}(n)$-structure on a $\operatorname{Spin}^{c}(n)$ - or $S O(n)$-bundle $P \rightarrow X$ is given by an element of a cohomology group of $X$. And also the set of such structures, if non-empty, has a free and transitive action of a cohomology group of $X$ (this gives rise to a non-canonical correspondence).

\begin{tabular}{c|c|c} 
lifting problem & obstruction in & action of \\
\hline $\operatorname{Spin}(n) \rightarrow S O(n)$ & $H^{2}\left(\cdot ; \mathbb{Z}_{2}\right)$ & $H^{1}\left(\cdot ; \mathbb{Z}_{2}\right)$ \\
\hline $\operatorname{Spin}^{c}(n) \rightarrow \operatorname{SO}(n)$ & $H^{3}(\cdot ; \mathbb{Z})$ & $H^{2}(\cdot ; \mathbb{Z})$ \\
\hline $\operatorname{Spin}(n) \rightarrow \operatorname{Spin}^{c}(n)$ & $H^{2}(\cdot ; \mathbb{Z})$ & $H^{1}(\cdot ; \mathbb{Z})$
\end{tabular}

The obstruction for $\operatorname{Spin}(n) \rightarrow S O(n)$ is given by the second StiefelWhitney class $w_{2}$, the obstruction for $\operatorname{Spin}^{c}(n) \rightarrow S O(n)$ is given by the third integral Stiefel-Whitney class $W_{3}$, which is the image of $w_{2}$ under the Bockstein boundary map. This means that a $\operatorname{Spin}^{c}(n)$-structure exists iff the obstruction against $\operatorname{Spin}(n)$-structures has an integral lift $\widetilde{w}_{2} \in H^{2}(\cdot ; \mathbb{Z})$.

Remark 2.2.5. The action of the cohomology groups on the sets of Spinand $S_{\text {pin }}{ }^{c}$-structures can also be described within the classical definition of $G$-structures (see [LM89] for $\operatorname{Spin}(n) \rightarrow S O(n)$ and [Fri00] for $\operatorname{Spin}^{c}(n) \rightarrow$ $S O(n))$. For example, if $a \in H^{1}\left(X ; \mathbb{Z}_{2}\right)$ is the pullback of the generator of $H^{1}\left(S O(n) ; \mathbb{Z}_{2}\right)$ under some map $f: X \rightarrow S O(n)$, then the action of $a$ on the set of $\operatorname{Spin}(n)$-structures is just post-composition with the bundle 
automorphism $P_{S O(n)} \rightarrow P_{S O(n)}$ given by $f$. This situation necessarily occurs when $\operatorname{dim}(X) \leq n$ : In this case $a$ is the pullback of the generator of $H^{1}\left(\mathbb{R} P^{n} ; \mathbb{Z}_{2}\right)$ under some map, and this generator is the pullback of the generator of $H^{1}\left(S O(n) ; \mathbb{Z}_{2}\right)$ under the map $\phi: \mathbb{R} P^{n}=S^{n} / \mathbb{Z}_{2} \rightarrow S O(n)$ where $\phi$ sends $q \in S^{n}$ to the linear map $\mathbb{R}^{n} \rightarrow \mathbb{R}^{n}$ that consists of reflection along the hyperplane perpendicular to $q$ followed by reflection along some fixed hyperplane.

In particular, it follows that for $\operatorname{dim}(X) \leq n$ representatives $P_{\operatorname{Spin}(n)}$, $P_{S \sin (n)}^{\prime}$ of different $\operatorname{Spin}(n)$-structures must be isomorphic as abstract $\operatorname{Spin}(n)$ bundles, and the only difference is in the map to $P_{S O(n)}$.

\subsection{Bordism and KO-homology}

\section{Spin- and Spin $^{c}$-bordism}

We give a short review of bordism groups. For a detailed account see [Koc96].

For the classes of groups $B(n)=O(n), S O(n), \operatorname{Spin}(n), \operatorname{Spin}^{c}(n)$ there are canonical inclusions $B(n) \rightarrow B(n+1) \rightarrow \ldots$ that commute with the group homomorphisms $B(n) \rightarrow O(n)$. For every principal $B(n)$-bundle $P_{B(n)} \rightarrow M$ these inclusions induce inclusions of principal bundles $P_{B(n)} \rightarrow P_{B(n+1)} \rightarrow \ldots$ A stable $B$-structure on a principal $O(n)$-bundle is represented by a $B(n+k)$ structure on the induced $O(n+k)$-bundle for some $k$. Two representatives are stably equivalent if the induced $B\left(n+k+k^{\prime}\right)$-structures are equivalent for some $k^{\prime}$.

In the homotopy theoretic picture this definition of stable $B$-structures translates as an equivalence class of lifts of the classifying map $M \rightarrow \mathbf{B} O(n) \rightarrow$ $\mathbf{B} O(n+1) \rightarrow \ldots$ along the sequence of fibrations

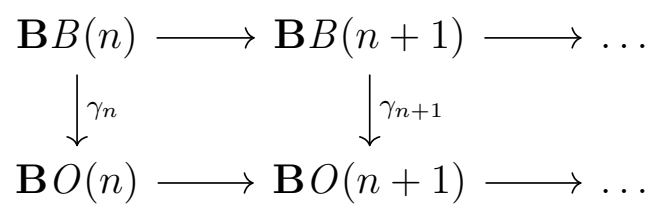

Stable Spin- and Spin $^{c}$-structures are therefore examples of (multiplicative) stable $(B, \gamma)$-structures as defined by Lashof.

A $B$-structure on a smooth manifold $M$ is defined as a stable $B$-structure on the principal bundle of orthonormal frames in the normal bundle $\nu(M)$ of any embedding $i: M \rightarrow \mathbb{R}^{N}$. It is non-trivial but true that this definition does not depend on the choice of the embedding. If $M$ has a boundary, then we can arrange that $i(M)$ is contained in the half-space $\left\{x_{N} \geq 0\right\} \subset \mathbb{R}^{N}$ and $i(\partial M)$ is contained in the hyperplane $\mathbb{R}^{N-1}=\left\{x_{N}=0\right\} \subset \mathbb{R}^{N}$. Then $\nu(M)_{\mid \partial M}$ is identified with the normal bundle of $\partial M$ inside $\mathbb{R}^{N-1}$ and a stable $B$-structure on $M$ defines by restriction a stable $B$-structure on $\partial M$.

Definition 2.3.1. Let $X$ be a topological space. 
A closed singular manifold with $B$-structure $f:\left(M, b_{M}\right) \rightarrow X$ is nullbordant if there exists a compact manifold with boundary $W$, a $B$-structure $b_{W}$ on $W$ and a singular map $F: W \rightarrow X$ such that $\partial W=M, b_{W}$ restricts to $b_{M}$ on $\partial W$ and $F$ restricts to $f$ on $\partial W$.

The $B$-bordism groups $\Omega_{k}^{B}(X)$ are defined as the bordism-equivalence classes of singular $k$-manifolds with $B$-structure $\left(f:\left(M, b_{M}\right) \rightarrow X\right)$. The group addition is disjoint union.

Remark 2.3.2. By the 2-out-of-3 principle one could equivalently define $B$ structures using the stable tangent bundle. Furthermore, in the case of $B=$ $S O, \operatorname{Spin}_{\text {Spin }}{ }^{c}$ it follows from the description of the obstruction against and classification of Spin- and Spin $^{c}$-structures in terms of cohomology classes that $B$-structures on the stable tangent bundle correspond to $B$-structures on the non-stabilized tangent bundle. This simplifies the definition of $B$-bordism in the case of $B=S O$, Spin, Spinc .

\section{The $K O$-fundamental class of a Spin-structure}

When $M$ is a manifold with a given Spin-structure, one can construct the corresponding Dirac differential operator which then gives rise to a fundamental class in the real K-homology of $M$. This is described in [HR00]. We give a short recapitulation.

The Clifford algebra $C l_{0, n}$ of a real Euclidean vector space $V=\mathbb{R}^{n}(n=$ $\operatorname{dim}(M))$ is the algebra generated by $V$ subject to the relations $v \cdot v=-\|v\|^{2}$. There is an embedding $\rho: \operatorname{Spin}(n) \rightarrow C l_{0, n}{ }^{\times}$of the spin group into the group of invertible elements of the Clifford algebra. $\operatorname{Spin}(n)$ then acts on the Clifford algebra by conjugation. On $V \subset C l_{0, n}$ this action coincides with the action $\operatorname{Spin}(n) \rightarrow S O(n) \curvearrowright V$.

Let now $s: P_{\text {Spin }} \rightarrow P_{S O}$ be a Spin-structure on the tangent bundle of $M$, and let $C l(M)$ be the Clifford-algebra bundle associated to the conjugation action of Spin. Inside $C l(M)$ sits the vector bundle $P_{\text {Spin }} \times_{\text {Spin }} V$ and the Spin-structure $s$ defines an isomorphism $P_{\text {Spin }} \times_{\text {Spin }} V \cong P_{S O} \times_{S O} V=T M$. Therefore, we can think of $T M$ as a sub-vector bundle of $C l(M)$. Now let the spinor bundle $\mathcal{S} \rightarrow M$ be the vector bundle associated to $P_{\text {Spin }}$ via the leftregular action Spin $\curvearrowright C l_{0, n}$. The fibers of $\mathcal{S}$ are left and right modules for the Clifford algebra. And the left-regular and conjugation actions fit together in just the right way that there is a well-defined left action of the algebra bundle $C l(M)$ on $\mathcal{S}$.

The Clifford algebra can be given a scalar product such that the left-regular representation of Spin is unitary. This induces a metric on $\mathcal{S}$. Furthermore, when a Riemannian metric is given on $M$, the Levi-Civita connection defines a principal connection on the bundle $P_{S O}$. Then there is a unique lift to a principal connection on $P_{\text {Spin }}$ which in turn induces a connection on $\mathcal{S}$. The Dirac operator corresponding to the Spin-structure $s$ is the elliptic first-order 
differential operator $\not D: \Gamma(M, \mathcal{S}) \rightarrow \Gamma(M, \mathcal{S})$ given by

$$
\not D(u)(x)=\sum_{i=1}^{n} e_{i} \cdot \nabla_{e_{i}} u(x)
$$

where $\left\{e_{i}\right\}_{\leq n}$ is any orthonormal basis of $T_{x} M$.

$\not D$ commutes with the right action of $C l_{0, n}$ on $\mathcal{S}$. When $M$ is complete with regard to the Riemannian metric, $D$ can be extended to an unbounded self-adjoint operator on $L^{2}(M, \mathcal{S})$. Using functional calculus it can then be turned into a bounded operator $\chi(\not D)$ that commutes with the multiplication operators of $C_{0}(M)$ up to compact operators and therefore defines an element $\left[C_{0}(M) \curvearrowright L^{2}(M, \mathcal{S}), \chi(\not D)\right] \in K O_{n}(M)$ in the Fredholm-module picture of real K-homology. This element behaves similarly to the fundamental class $[M] \in H_{n}(M ; \mathbb{Z})$ of an oriented manifold. We call it the KO-fundamental class and denote it by $[M]_{K O}$. The class $[M]_{K O}$ depends on the differential structure of $M$ and on the choice of the Spin-structure. It does not depend on the Riemannian metric.

\section{The natural transformation $\Omega^{\text {Spin }} \rightarrow K O$}

The construction of the fundamental class $[M]_{K O}$ corresponding to a Spinstructure is compatible with taking boundaries: If $\left(W, s_{W}\right)$ is a compact Spinmanifold with boundary $\left(M, s_{M}\right)$ and $i: M \rightarrow W$ is the inclusion, then $i_{*}\left([M]_{K O}\right)=0 \in K O(M)$. Therefore, one can construct a natural transformation $\Omega_{*}^{\text {Spin }}(\cdot) \rightarrow K O_{*}(\cdot)$ by the assignment $[f:(M, s) \rightarrow X] \mapsto f_{*}\left([M]_{K O}\right)$ where $[M]_{K O}$ is determined by $s$.

This transformation gets even simpler when one uses the geometric picture of K-homology due to Baum and Douglas [BD82, which is equivalent to the definition via Fredholm modules (see [BHS07]).

Definition 2.3.3. Let $X$ be a topological space. Elements of $K O_{n}(X)$ are represented by quadruples $(M, s, E, f)$ where $M$ is a closed smooth manifold of dimension $n \bmod 8, s$ a Spin-structure on $M, E \rightarrow M$ a real vector bundle and $f: M \rightarrow X$ a continuous map. Two quadruples represent the same element of $K O_{n}(X)$ if they are equivalent under the equivalence relation generated by the following rules:

1. Direct sum of vector bundles:

$$
\left(M \sqcup M, s \sqcup s, E_{1} \sqcup E_{2}, f \sqcup f\right) \sim\left(M, s, E_{1} \oplus E_{2}, f\right)
$$

2. Bordism:

$\left(M_{1}, s_{1}, E_{1}, f_{1}\right) \sim\left(M_{2},-s_{2}, E_{2}, f_{2}\right)$ whenever there is a manifold with boundary $W$, with a Spin-structure $s_{W}$, vector bundle $E_{W} \rightarrow W$ and map $W \rightarrow X$ such that $\partial W \cong M_{1} \sqcup M_{2}$ and $s_{1,2}, E_{1,2}, f_{1,2}$ is the induced structure $\left(-s_{2}\right.$ is obtained from $s_{2}$ by reversing the orientation). 
3. "Vector bundle modification":

For any 8-dimensional vector bundle $\pi: H \rightarrow M$ with a Spin-structure $s_{H}$ it holds $(M, s, E, f) \sim\left(Z, s_{Z}, F \otimes \pi^{*} E, f \circ \pi\right)$ where $Z$ is the unit sphere bundle inside $H \oplus(M \times \mathbb{R}), s_{Z}$ is determined by $s$ and $s_{H}$, and $F$ is the reduced spinor bundle obtained from the Spin-structure $s_{H}$ (see [BHS07]). This rule enforces Bott periodicity.

The addition is disjoint union.

With this definition of K-homology the natural transformation sends the class $[M, s, f] \in \Omega^{\operatorname{Spin}}(X)$ to $[M, s, M \times \mathbb{R}, f] \in K O(X)$.

\subsection{The index-theoretical obstruction}

We now give an overview over the classical results about index-theoretical obstructions. More information can be found in the survey [RS01].

The basis for the index-theoretical obstructions against positive scalar curvature is the Lichnerowicz-Schrödinger-Weitzenböck formula:

Theorem 2.4.1. Let $M$ be a Riemannian manifold with Spin-structure, $\mathcal{S} \rightarrow$ $M$ the corresponding spinor bundle, $\nabla: \Gamma(M, \mathcal{S}) \rightarrow \Gamma\left(M, T^{*} M \otimes \mathcal{S}\right)$ the connection induced by the metric on $M$ and $\not D: \Gamma(M, \mathcal{S}) \rightarrow \Gamma(M, \mathcal{S})$ the Dirac operator.

Then it holds

$$
\not D^{2}=\nabla^{*} \nabla+\frac{1}{4} \kappa
$$

where $\nabla^{*}$ is the formal adjoint and $\kappa$ is the scalar curvature function on $M$.

In particular, if $M$ has a metric of positive scalar curvature, then $\not D^{2}$ has a spectral gap at 0 and therefore $\not D$ is invertible. From the Fredholm-module picture of K-homology it then follows that $q_{*}\left([M]_{K O}\right)=0 \in K O_{n}(p t)$ where $q$ is the collapse map. The other way around, this means that $q_{*}\left([M]_{K O}\right)$ is an obstruction against positive scalar curvature on $M$.

This obstruction can be combined with the positive results obtained from surgery. Stolz showed ([Sto92]):

Theorem 2.4.2. Let $M$ be a simply connected Spin manifold of dimension $\geq 5$.

Then $M$ is psc if and only if $q_{*}\left([M]_{K O}\right)=0 \in K O_{n}(p t)$.

In the non-simply connected case the obstruction $q_{*}\left([M]_{K O}\right)=0 \in K O_{n}(p t)$ is too crude. For example, the torus $M=\mathbb{T}^{3}$ is Spin-null bordant, and this implies $q_{*}\left([M]_{K O}\right)=0$. The solution is to replace $K O(p t)$ with the K-theory of the (real) group $C^{*}$-algebra, $K O\left(C^{*}\left(\pi_{1}(M)\right)\right)$ :

Using the Mishchenko-Fomenko bundle and the biproduct of KK-theory one can construct an assembly map $K O\left(\mathbf{B} \pi_{1}(M)\right) \rightarrow K O\left(C^{*}\left(\pi_{1}(M)\right)\right.$ ) (see 
[Kas88, 6.2]). This map can be precomposed with the morphism induced by a classifying map $u_{M}: M \rightarrow \mathbf{B} \pi_{1}(M)$. Including the natural transformation $\Omega^{\text {Spin }} \rightarrow K O$ we get the following diagram:

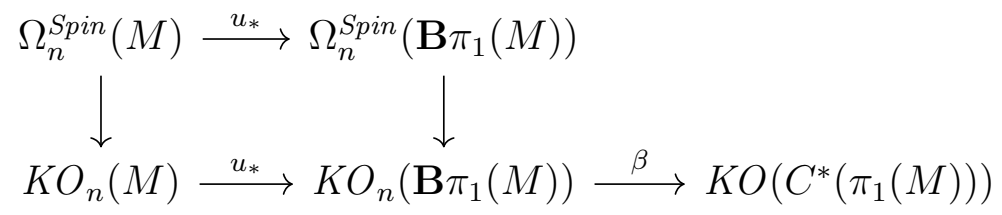

The natural transformation $\Omega^{\text {Spin }} \rightarrow K O$ also factors through the connective covering $k o$ of the homology theory $K O$, and the assembly map $\beta$ factors through the Baum-Connes map $K O^{\pi_{1}(M)}\left(\underline{\mathbf{E}} \pi_{1}(M)\right) \rightarrow K O\left(C^{*}\left(\pi_{1}(M)\right)\right)$.

We call the image of the fundamental class $[M]_{\text {Spin }}=[M, s, \mathrm{id}] \in \Omega^{\operatorname{Spin}}(M)$ under the concatenation $\alpha: \Omega^{\operatorname{Spin}}(M) \rightarrow K O\left(C^{*}\left(\pi_{1}(M)\right)\right)$ the Rosenberg index of $M$. Intuitively speaking, it is obtained by forming a $C^{*}\left(\pi_{1}(M)\right)$-bundle over $M$, with a twist given by the left-regular action $\pi_{1}(M) \curvearrowright C^{*}\left(\pi_{1}(M)\right)$, and then taking the (graded) kernel of the Dirac operator twisted with the $C^{*}$-algebra bundle.

Using the fact that the $C^{*}\left(\pi_{1}(M)\right)$-bundle is flat, Rosenberg refined the argument of Lichnerowicz-Schrödinger-Weitzenböck and showed that the Rosenberg index $\alpha\left([M]_{\text {Spin }}\right) \in K O_{n}\left(C^{*}\left(\pi_{1}(M)\right)\right)$ is an obstruction against positive scalar curvature.

Remark 2.4.3. We have tacitly assumed - and will continue to assume - that the classifying map $u: M \rightarrow \mathbf{B} \pi_{1}(M)$ is fixed at least up to homotopy. If we replace $u$ by another map $u^{\prime}$ that also induces an isomorphism of fundamental groups, then up to homotopy $u^{\prime}$ factors as $u^{\prime}=a_{\phi} \circ u$ where $a_{\phi}: \mathbf{B} \pi_{1}(M) \rightarrow$ $\mathbf{B} \pi_{1}(M)$ is induced by a group automorphism $\phi: \pi_{1}(M) \rightarrow \pi_{1}(M)$. By naturality of the assembly map it then holds

$$
\beta \circ u_{*}^{\prime}\left([M]_{K O}\right)=\beta \circ a_{\phi_{*}} \circ u_{*}\left([M]_{K O}\right)=\Phi_{*} \circ \beta \circ u_{*}\left([M]_{K O}\right),
$$

where $\Phi: C^{*}\left(\pi_{1}(M)\right) \rightarrow C^{*}\left(\pi_{1}(M)\right)$ is the $C^{*}$-algebra automorphism induced by $\phi$. In particular, the vanishing of $\alpha\left([M]_{\text {Spin }}\right)$ does not depend on the choice of $u$.

The Rosenberg index also depends on the choice of the Spin-structure. This will be discussed in Section 3 .

Remark 2.4.4. The $C^{*}$-algebra $C^{*}\left(\pi_{1}(M)\right)$ can be taken to be either the maximal or the reduced group $C^{*}$-algebra, the Rosenberg index can be constructed in both cases. The Baum-Connes conjecture $K O^{\pi_{1}(M)}\left(\underline{\mathbf{E}} \pi_{1}(M)\right) \cong$ $K O\left(C^{*}\left(\pi_{1}(M)\right)\right)$ assumes that the reduced $C^{*}$-algebra is used.

On the other hand, while every group homomorphism induces a homomorphism of maximal group $C^{*}$-algebras, this is not always the case for the reduced $C^{*}$-algebras (it $i s$ true if the kernel of the group homomorphism is 
amenable, which is the case for the homomorphisms we will consider in the following sections). Furthermore, the reduced version of the Rosenberg index factors through the induced map of the canonical quotient map $C_{\max }^{*}\left(\pi_{1}(M)\right) \rightarrow$ $C_{\text {red }}^{*}\left(\pi_{1}(M)\right)$ and therefore will never contain more information than the maximal version.

We will take $C^{*}\left(\pi_{1}(M)\right)$ to be the maximal group $C^{*}$-algebra, if not indicated otherwise.

The Rosenberg index is a very effective obstruction. Indeed, Rosenberg and Stolz showed ([RS01], 4.13):

Theorem 2.4.5. Let $M$ be a closed connected Spin manifold of dimension $\geq 5$ and $u_{M}: M \rightarrow \mathbf{B} \pi_{1}(M)$ a classifying map.

Assume that the assembly map $K O\left(\mathbf{B} \pi_{1}(M)\right) \rightarrow K O\left(C^{*}\left(\pi_{1}(M)\right)\right)$ is injective, and that the map $k o\left(\mathbf{B} \pi_{1}(M)\right) \rightarrow K O\left(\mathbf{B} \pi_{1}(M)\right)$ is injective.

Then $M$ is psc if and only if $\alpha\left([M]_{\text {Spin }}\right)=0 \in K O_{n}\left(C^{*}\left(\pi_{1}(M)\right)\right)$.

For general fundamental groups the statement is wrong. In Sch98 Schick constructs a counterexample where the Rosenberg index vanishes but the minimal hypersurface method shows that the manifold is not psc.

One reason why Theorem 2.4.5 may fail - especially in low dimensions is Bott periodicity. If $B t$ is an 8-dimensional simply-connected Spin manifold with $\widehat{A}(B t)=1$, then $\alpha\left([M \times B t]_{\text {Spin }}\right)=\alpha\left([M]_{\text {Spin }}\right)$. But it may happen that $M \times B t$ is psc and $M$ is not. This motivates a "stable" version of Theorem 2.4.5. Stolz showed ([Sto02]):

Theorem 2.4.6. Let $M$ be a Spin manifold and $u_{M}: M \rightarrow \mathbf{B} \pi_{1}(M)$ a classifying map. Let Bt be a simply connected Spin manifold of dimension 8 with $\widehat{A}(B t)=1$. Assume that the Baum-Connes map $K O_{*}^{\pi_{1}(M)}\left(\underline{\mathbf{E}} \pi_{1}(M)\right) \rightarrow$ $K O_{*}\left(C_{\text {red }}^{*}\left(\pi_{1}(M)\right)\right)$ is injective.

Then $\alpha\left([M]_{\text {Spin }}\right)=0 \in K O\left(C^{*}\left(\pi_{1}(M)\right)\right)$ if and only if the product manifold $M \times B t \times \cdots \times B t$ allows a psc metric for sufficiently many Bt-factors.

If the manifold $M$ is not Spin but has a $\operatorname{Spin}^{c}$-structure, there is still a Dirac operator that defines a fundamental class in the complex K-homology group $K_{n}(M)$. Indeed, the group $\operatorname{Spin}^{c}$ embeds into the group of invertible elements inside the complexification of the Clifford algebra $C l_{0, n} \otimes_{\mathbb{R}} \mathbb{C}$. This can be used to construct a complex spinor bundle and a Dirac operator on this bundle.

One important difference to the real case is that the connection on the complex spinor bundle is not uniquely determined by the Riemannian metric on $M$. Instead, it is determined uniquely only when one fixes in addition to the LeviCivita connection on $M$ a principal connection on the $S^{1}$-bundle determined by the Spin $^{c}$-structure. The analog of the Lichnerowicz-Schrödinger-Weitzenböck formula then takes the following form ([LM89] D.12): 
Theorem 2.4.7. Let $M$ be a Riemannian manifold with Spin ${ }^{c}$-structure and $\mathcal{S} \rightarrow M$ the corresponding spinor bundle. Let further $\omega$ be the curvature 2form of a fixed connection of the $S^{1}$-bundle associated to the Spin ${ }^{c}$-structure, let $\nabla: \Gamma(M, \mathcal{S}) \rightarrow \Gamma\left(M, T^{*} M \otimes \mathcal{S}\right)$ be the induced connection on the spinor bundle and $\not D: \Gamma(M, \mathcal{S}) \rightarrow \Gamma(M, \mathcal{S})$ the Dirac operator.

Then

$$
\not D^{2}=\nabla^{*} \nabla+\frac{1}{4} \kappa+\frac{i}{2} \omega,
$$

where $\kappa$ is the scalar curvature function on $M$ and $\omega$ denotes Clifford multiplication by the 2-form $\omega$.

In the special case where the $S^{1}$-bundle associated to the $S p i n^{c}$-structure is classified by a torsion element in $H^{2}(M ; \mathbb{Z})$, the $S^{1}$-bundle can be given a flat connection and the fundamental class $[M]_{K}$ can be used to construct an obstruction against positive scalar curvature as before.

In Section 4 we will try a different approach where no control over the connection of the $S^{1}$-bundle is assumed and instead its fundamental group becomes important. 


\section{The effect of changing the Spin structure}

Let $M$ be a Spin manifold with fundamental group $\Gamma$. Different choices of Spin-structures on $M$ lead to different fundamental classes $[M]_{\operatorname{Spin}} \in \Omega_{n}^{\operatorname{Spin}}(M)$ and $[M]_{K O} \in K O_{n}(M)$. This leads to the question how the image $\alpha\left([M]_{\text {Spin }}\right)$ behaves when the spin structure is changed. In particular, is it possible that the obstruction vanishes for one spin structure, but not for another?

Already in Ros86] it was noted that the answer to the last question is no. The idea is that the group $H^{1}\left(M ; \mathbb{Z}_{2}\right)$ acts on the set of Spin-fundamental classes as well as on the group $K O_{n}\left(C^{*}(\Gamma)\right)$, and $\alpha$ intertwines the actions. Therefore, if the spin structures $s_{1}$ and $s_{2}$ are related by $x . s_{1}=s_{2}, x \in$ $H^{1}\left(M ; \mathbb{Z}_{2}\right)$, then $\alpha\left([M]_{s_{1}}\right)=0$ implies $\alpha\left([M]_{x . s_{1}}\right)=x . \alpha\left([M]_{s_{1}}\right)=x .0=0$. In [Ros86] all details of this argument are omitted. In this section we will use the calculus of KK-theory to explicitly calculate the effect that a change of Spin-structures has on the Rosenberg index.

Let $M$ be a closed connected smooth Spin manifold of dimension $n$, and let the orientation of $M$ be fixed. Let $s_{1}, s_{2}: P_{\text {Spin }} \rightarrow P_{S O}$ be representatives of two Spin-structures on TM. As explained in Remark 2.2.5, $s_{2}$ can be obtained from $s_{1}$ by postcomposing with an $S O$-bundle automorphism which is given by a map $a_{x}: M \rightarrow S O(n)$. This map determines a group homomorphism $\varphi_{x}: \Gamma \rightarrow \pi_{1}(S O) \rightarrow \mathbb{Z}_{2}=\{ \pm 1\} \subset \mathbb{R}$. Via the isomorphism

$$
\begin{aligned}
\operatorname{Hom}\left(\Gamma, \mathbb{Z}_{2}\right) & \cong \operatorname{Hom}\left(\frac{\Gamma}{[\Gamma, \Gamma]}, \mathbb{Z}_{2}\right) \\
& \cong \operatorname{Hom}\left(H_{1}(M, \mathbb{Z}) ; \mathbb{Z}_{2}\right) \cong H^{1}\left(M ; \mathbb{Z}_{2}\right)
\end{aligned}
$$

it also determines an element $x \in H^{1}\left(M ; \mathbb{Z}_{2}\right)$, and since the Eilenberg-MacLane space $K\left(\mathbb{Z}_{2}, 1\right)$ classifies $\mathbb{Z}_{2}$-bundles, this element defines a principal $\mathbb{Z}_{2}$-bundle $Y_{x} \rightarrow M$.

Following the construction of the Dirac operators as in Section 2.3 we see that the spinor bundles $\mathcal{S}_{1}, \mathcal{S}_{2}$ determined by the two Spin-structures are canonically isomorphic as vector bundles with a right Clifford action. The difference lies in the Clifford-actions $\lambda_{1 / 2}: T M \rightarrow \operatorname{End}\left(\mathcal{S}_{1 / 2}\right)$. Under the identification $\mathcal{S}_{1}=\mathcal{S}_{2}$ as vector bundles, $\lambda_{1}=\lambda_{2} \circ \Psi\left(a_{x}\right)$ where $\Psi\left(a_{x}\right): T M \rightarrow T M$ is the vector bundle automorphism given by $a_{x}$. Passing to the universal covering $\widetilde{M}$ we have the tangent bundle $\widetilde{T M}=T M \times_{M} \widetilde{M}$, sitting inside the Clifford algebra bundle $\widetilde{C l(M)}=C l(M) \times_{M} \widetilde{M}$, and the two Clifford module bundles $\widetilde{\mathcal{S}_{1 / 2}}=\mathcal{S}_{1 / 2} \times_{M} \widetilde{M}$, with $\Gamma$ acting on all of them. The lifted actions $\widetilde{\lambda_{1 / 2}}$ differ by precomposition with $\Psi\left(a_{x} \circ p_{\widetilde{M}}\right)$. But $a_{x} \circ p_{\widetilde{M}}: \widetilde{M} \rightarrow M \rightarrow S O(n)$ has a lift $\eta: M \rightarrow \operatorname{Spin}(n) \subset C l\left(\mathbb{R}^{n}\right)$, which satisfies $\gamma \cdot \eta=\varphi_{x}(\gamma) \cdot \eta$ for $\gamma \in \Gamma$. 
Let now $T: \widetilde{\mathcal{S}_{1}} \rightarrow \widetilde{\mathcal{S}_{2}}$ be given by the fiberwise Clifford multiplication with $\eta^{-1}$ from the left. Then $T$ is a bundle isomorphism, compatible with the right Clifford action, and it intertwines the actions $\lambda_{1 / 2}$. Indeed, if $i_{2}: \widetilde{T M} \rightarrow \widetilde{C l(M)}$ is the inclusion from the second lifted Spin-structure and $\star$ denotes Clifford multiplication, then for $m \in \widetilde{M}, \xi \in \widetilde{T M}_{m}, \zeta \in \widetilde{\mathcal{S}}_{1}$ :

$$
\begin{aligned}
\lambda_{1}(\xi)(\zeta) & =\lambda_{2}\left(a_{x}\left(p_{\widetilde{M}}(m)\right) \cdot \xi\right)(\zeta) \\
& =i_{2}\left(a_{x}\left(p_{\widetilde{M}}(m)\right) \cdot \xi\right) \star \zeta \\
& =\eta(m) \star i_{2}(\xi) \star \eta(m)^{-1} \star \zeta \\
& =T^{-1}\left(i_{2}(\xi) \star T(\zeta)\right) \\
& =\left(T^{-1} \circ \lambda_{2}(\xi) \circ T\right)(\zeta) .
\end{aligned}
$$

By quotienting out the $\Gamma$-action we obtain $\mathcal{S}_{2} \cong Y_{x} \times_{\mathbb{Z}_{2}} \mathcal{S}_{1}$ as spinor bundles with left Clifford action by $T M$ and right action by $C l\left(\mathbb{R}^{n}\right)$ (The twist is the result of the twisted equivariance of $\eta$ and $T$ ).

Since $\mathbb{Z}_{2}$ is discrete, the connection on $\mathcal{S}_{1}$ induces a connection on $\mathcal{S}_{2}=$ $Y_{x} \times_{\mathbb{Z}_{2}} \mathcal{S}_{1}$. This is a Dirac connection in the sense of [HR00, 11.1.9.] and can therefore be used to construct the Dirac operator representing $[M]_{2} \in$ $K O_{n}(M)$.

Instead of twisting $\mathcal{S}_{1}$ with $Y_{x}$ we can also construct the $\mathbb{R}$-line bundle $Z_{x}=Y_{x} \times_{\mathbb{Z}_{2}} Z_{0}$ by twisting the trivial line bundle $Z_{0}=\mathbb{R} \times M$ with $Y_{x}$, and we can write

$$
\mathcal{S}_{2}=Y_{x} \times_{\mathbb{Z}_{2}} \mathcal{S}_{1}=Y_{x} \times_{\mathbb{Z}_{2}}\left(Z_{0} \otimes \mathcal{S}_{1}\right)=\left(Y_{x} \times_{\mathbb{Z}_{2}} Z_{0}\right) \otimes \mathcal{S}_{1}=Z_{x} \otimes \mathcal{S}_{1} .
$$

We will use the following classes in (real) KK-Theory:

- $[M]_{1} \in K K\left(C(M), C l_{0, n}\right)$ is represented by the (normalized) Dirac operator $\chi\left(D_{1}\right)$ acting on the first spinor bundle $\mathcal{S}_{1}$.

- $[M]_{2} \in K K\left(C(M), C l_{0, n}\right)$ is represented by the (normalized) Dirac operator $\chi\left(\not D_{2}\right)$ acting on the modified spinor bundle $\mathcal{S}_{2}$.

- $\beta \in K K\left(\mathbb{R}, C(M) \hat{\otimes} C^{*}(\Gamma)\right)$ is represented by the zero operator on the Hilbert module $\mathcal{F}$ of continuous sections from $M$ into the $C^{*}(\Gamma)$-bundle twisted by the fundamental group action, i.e.,

$$
\mathcal{F}=\left\{f \in C\left(\widetilde{M}, C^{*}(\Gamma)\right) \mid f(g . x)=g . f(x) \forall g \in \Gamma\right\} .
$$

- $\phi \in K K\left(C^{*}(\Gamma), C^{*}(\Gamma)\right)$ is represented by the zero operator on $C^{*}(\Gamma)$ viewed as a Hilbert module over itself. The left action is given by $\Phi: C^{*}(\Gamma) \rightarrow C^{*}(\Gamma), \delta_{g} \mapsto \varphi_{x}(g) \cdot \delta_{g} .\left(\right.$ Note that $\left.\Phi^{-1}=\Phi\right)$

- $z \in K K(C(M), C(M))$ is represented by the zero operator on the Hilbert module $\mathcal{Z}$ of continuous sections from $M$ into the line bundle $Z_{x}$, i.e.,

$$
\mathcal{Z}=\{f \in C(\widetilde{M}, \mathbb{R}) \mid f(g x)=\phi(g) f(x), g \in \Gamma\} .
$$


Finally, for any $\mathrm{C}^{*}$-algebras $A, B, D$ let

$$
\tau_{D}: K K(A, B) \rightarrow K K(A \hat{\otimes} D, B \hat{\otimes} D)
$$

be given by tensoring the Hilbert module with $D$ and the operator with id: $D \rightarrow D$. With this notation

$$
\alpha\left([M]_{1 / 2}\right)=\beta \hat{\otimes}_{C(M) \hat{\otimes} C^{*}(\Gamma)} \tau_{C^{*}(\Gamma)}\left([M]_{1 / 2}\right)
$$

(see [Kas88, 6.2]).

The following is a special case of twisting the KK-class of a differential operator with a vector bundle:

Lemma 3.1. Let $[M]_{1},[M]_{2}$, $z$ be as above, then it holds

$$
[M]_{2}=z \hat{\otimes}_{C(M)}[M]_{1} .
$$

Proof. Working with the representatives above, we show that $\chi\left(D_{2}\right)$ acting on $L^{2}\left(M, \mathcal{S}_{2}\right)$ represents the Kasparov product of $z$ and $[M]_{1}$, as in definition 18.4.1 in Bla98.

As Hilbert bimodules, $L^{2}\left(M, \$_{2}\right)=\mathcal{Z} \hat{\otimes}_{C(M)} L^{2}\left(M, \$_{1}\right)$. Also, the representative of $z$ has the zero operator and $\chi\left(\not D_{2}\right)$ is a Fredholm operator, so we just have to check that $\chi\left(\not D_{2}\right)$ is a $\chi\left(\not D_{1}\right)$-connection in the sense of [Bla98, 18.3.1], i.e., that for any $\xi \in \mathcal{Z}$

$$
\begin{aligned}
T_{\xi} \cdot \chi\left(\not D_{1}\right)-\chi\left(\not D_{2}\right) \cdot T_{\xi} & \in \mathbb{K}\left(L^{2}\left(M, \mathcal{S}_{1}\right), L^{2}\left(M, \mathcal{S}_{2}\right)\right), \\
\chi\left(\not D_{1}\right) \cdot T_{\xi}^{*}-T_{\xi}^{*} \cdot \chi\left(\not D_{2}\right) & \in \mathbb{K}\left(L^{2}\left(M, \mathcal{S}_{2}\right), L^{2}\left(M, \mathcal{S}_{1}\right)\right),
\end{aligned}
$$

where $T_{\xi}(y)=\xi \hat{\otimes} y$ and $T_{\xi}^{*}\left(y \hat{\otimes} y^{\prime}\right)=\langle y, \xi\rangle y^{\prime}$.

We partition $M$ into a finite number of pieces $P_{i}$, such that each piece has an open neighborhood $U_{i}$ over which $Z_{x}$ can be trivialized in a way that is compatible with the connection. This trivialization is unique up to a sign and determines a unitary equivalence of $L^{2}\left(U, \mathcal{S}_{1}\right)$ and $L^{2}\left(U, \mathcal{S}_{2}\right)$. The equivalence intertwines the actions of $C(M)$. Furthermore it identifies $\not_{1 \mid U_{i}}$ with $\not_{2 \mid U_{i}}$ and by $\left[\right.$ HR00, 10.8.4] it follows that also $\chi\left(\not D_{1}\right)_{\mid U_{i}}$ and $\chi\left(\not D_{2}\right)_{\mid U_{i}}$ are identified modulo compact operators. Because the operators $\chi\left(\not D_{1 / 2}\right)$ commute with the action of $C(M)$ up to compacts, they map $L^{2}$-functions supported in $P_{i}$ to $L^{2}$ functions supported in $U_{i}$ (modulo compacts). Finally, under the identification $L^{2}\left(U, \mathcal{S}_{1}\right)=L^{2}\left(U, \mathcal{S}_{2}\right)$ the operators $T_{\xi}$ and $T_{\xi}{ }^{*}$ restrict to multiplication with a function in $C\left(\overline{U_{i}}\right)$, determined by $\xi$. Since such multiplications commute with $\chi\left(\not D_{1 / 2}\right)$ up to compacts, it follows that the conditions above are satisfied when we restrict the domain of the operators to $L^{2}$-functions over a single piece $P_{i}$. Because the number of pieces is finite, this means that the conditions hold in general.

This shows that $\chi\left(\not D_{2}\right)$ acting on $L^{2}\left(M, \mathcal{S}_{2}\right)$ represents the Kasparov product of $z$ and $[M]_{1}$, concluding the proof. 
The next lemma contains the key observation of this computation.

Lemma 3.2. Let $\beta, \tau, x, \phi$ be as above, then

$$
\beta \hat{\otimes}_{C(M) \hat{\otimes} C^{*}(\Gamma)} \tau_{C^{*}(\Gamma)}(z)=\beta \hat{\otimes}_{C(M) \hat{\otimes} C^{*}(\Gamma)} \tau_{C(M)}(\phi) .
$$

Proof. Since the operators of the representatives on both sides are zero and the left action is just $\mathbb{R} \cdot i d$, it suffices to show that the $\left(C(M) \hat{\otimes} C^{*}(\Gamma)\right)$-Hilbert modules are unitarily equivalent. Both Hilbert modules have the trivial grading. The left one can be written as

$$
\mathcal{F} \hat{\otimes}_{C(M) \otimes C^{*}(\Gamma)}\left(\mathcal{Z} \otimes C^{*}(\Gamma)\right)=C^{\mu \otimes \lambda}\left(\widetilde{M}, C^{*}(\Gamma)\right) \otimes_{C(M) \otimes C^{*}(\Gamma)} C^{\mu \otimes \varphi}\left(\widetilde{M}, C^{*}(\Gamma)\right) .
$$

Here $C^{\cdot \otimes \cdot}\left(\widetilde{M}, C^{*}(\Gamma)\right)$ denotes the subset consisting of those bounded continuous functions in $C_{b}\left(\widetilde{M}, C^{*}(\Gamma)\right)=C_{b}(\widetilde{M}) \otimes C^{*}(\Gamma)$ that are invariant under the action of $\Gamma$ specified by $\cdot \otimes \cdot$. The actions used are $\lambda$ for left multiplication with $g, \varphi$ for multiplication with $\varphi(g), \operatorname{tr}$ for the trivial action and $\mu$ for translation, i.e., $(\mu(g) f)(\cdot)=f\left(g^{-1} \cdot\right)$.

The right Hilbert module can be written as

$$
\mathcal{F} \otimes_{C(M) \otimes C^{*}(\Gamma)}^{\Phi}\left(C(M) \otimes C^{*}(\Gamma)\right)=C^{\mu \otimes \lambda}\left(\widetilde{M}, C^{*}(\Gamma)\right) \otimes_{C(M) \otimes C^{*}(\Gamma)}^{\Phi} C^{\mu \otimes \operatorname{tr}}\left(\widetilde{M}, C^{*}(\Gamma)\right) .
$$

Here the $\Phi$ above the tensor is a reminder of the non-standard left action of $C^{*}(\Gamma)$ on the right hand module.

Both Hilbert modules are in fact unitarily equivalent to $C^{\mu \otimes \varphi \lambda}\left(\widetilde{M}, C^{*}(\Gamma)\right)$. The equivalences are given by

$$
\begin{aligned}
& u_{1}: C^{\mu \otimes \lambda}\left(\widetilde{M}, C^{*}(\Gamma)\right) \otimes_{C(M) \otimes C^{*}(\Gamma)} C^{\mu \otimes \varphi}\left(\widetilde{M}, C^{*}(\Gamma)\right) \rightarrow C^{\mu \otimes \varphi \lambda}\left(\widetilde{M}, C^{*}(\Gamma)\right), \\
& q \otimes r \mapsto q \cdot r, \\
& u_{2}: C^{\mu \otimes \lambda}\left(\widetilde{M}, C^{*}(\Gamma)\right) \otimes_{C(M) \otimes C^{*}(\Gamma)}^{\Phi} C^{\mu \otimes t r}\left(\widetilde{M}, C^{*}(\Gamma)\right) \rightarrow C^{\mu \otimes \varphi \lambda}\left(\widetilde{M}, C^{*}(\Gamma)\right) \text {, } \\
& q \otimes r \mapsto(\Phi \circ q) \cdot r .
\end{aligned}
$$

Both maps are well defined on the algebraic tensor product. To see that they indeed map into $C^{\mu \otimes \varphi \lambda}\left(\widetilde{M}, C^{*}(\Gamma)\right)$ we compute for $u_{1}$

$$
\begin{aligned}
(\mu(g) \otimes \varphi(g) \lambda(g))(q r)(y) & =\varphi(g) g \cdot q\left(g^{-1} y\right) \cdot r\left(g^{-1} y\right) \\
& =(\mu(g) \otimes \lambda(g))(q)(y) \cdot(\mu(g) \otimes \varphi(g))(r)(y) \\
& =q(y) \cdot r(y)=q r(y)
\end{aligned}
$$

and for $u_{2}$

$$
\begin{aligned}
(\mu(g) \otimes \varphi(g) \lambda(g))((\Phi q) \cdot r)(y) & =\varphi(g) g \cdot(\Phi q)\left(g^{-1} y\right) \cdot r\left(g^{-1} y\right) \\
& =\Phi(g) \cdot(\Phi q)\left(g^{-1} y\right) \cdot r\left(g^{-1} y\right) \\
& =\Phi\left(g \cdot q\left(g^{-1} y\right)\right) \cdot r\left(g^{-1} y\right) \\
& =\Phi((\mu(g) \otimes \lambda(g))(q)(y)) \cdot(\mu(g) \otimes \operatorname{tr}(g))(r)(y) \\
& =\Phi(q(y)) \cdot r(y)=((\Phi q) \cdot r)(y) .
\end{aligned}
$$


Both maps respect the right action of $C(M) \otimes C^{*}(\Gamma)$ and they respect the $\left(C(M) \otimes C^{*}(\Gamma)\right)$-valued scalar product. This means that they extend to an injective map on the $\mathrm{C}^{*}$-tensor product.

It just remains to check that the maps are surjective. Let an element $s \in C^{\mu \otimes \varphi \lambda}\left(\widetilde{M}, C^{*}(\Gamma)\right)$ be given. After applying a $\Gamma$-invariant partition of unity on $\widetilde{M}$, we may assume that $s$ is supported in a small $\Gamma$-neighborhood of a point, that can be trivialized as $U \times \Gamma$.

Now the function $q_{0}: U \times 1 \rightarrow C^{*}(\Gamma), q_{0}(u \times 1)=\delta_{1}$ can be extended to a function $q_{1} \in C^{\mu \otimes \lambda}\left(\widetilde{M}, C^{*}(\Gamma)\right)$. Define $r_{1}(y)$ to be $q_{1}(y)^{-1} \cdot s(y)$ on $\Gamma U$ and zero elsewhere. Then $u_{1}\left(q_{1} \otimes r_{1}\right)=s$. Also, $\Phi s \in C^{\mu \otimes \lambda}\left(\widetilde{M}, C^{*}(\Gamma)\right)$ and $u_{2}(\Phi s \otimes 1)=s$.

Finally, we piece it all together.

Theorem 3.3. Let $M$ be a connected closed smooth spin manifold with fixed orientation, let $s_{1}, s_{2}$ be two spin structures on $M$ and $[M]_{1},[M]_{2}$ the $\mathrm{KO}$ fundamental classes corresponding to these spin structures. Let $x \in H^{1}\left(M ; \mathbb{Z}_{2}\right)$ be the unique element that modifies $s_{1}$ into $s_{2}$ and let $\Phi=\Phi_{x}$ be the corresponding automorphism of $C^{*}(\Gamma)$.

Then

$$
\alpha\left([M]_{2}\right)=\Phi_{*}\left(\alpha\left([M]_{1}\right)\right) .
$$

Proof. To simplify notation we abbreviate $A=C(M) \hat{\otimes} C^{*}(\Gamma)$. We use the properties of the Kasparov product described in [Bla98, 18.9] to calculate:

$$
\begin{aligned}
\alpha\left([M]_{2}\right) & =\beta \hat{\otimes}_{A} \tau_{C^{*}(\Gamma)}\left([M]_{2}\right) \\
& =\beta \hat{\otimes}_{A} \tau_{C^{*}(\Gamma)}\left(z \hat{\otimes}_{C(M)}[M]_{1}\right) \\
& =\beta \hat{\otimes}_{A} \tau_{C^{*}(\Gamma)}(z) \hat{\otimes}_{A} \tau_{C^{*}(\Gamma)}\left([M]_{1}\right) \\
& \stackrel{a)}{=} \beta \hat{\otimes}_{A} \tau_{C(M)}(\phi) \hat{\otimes}_{A} \tau_{C^{*}(\Gamma)}\left([M]_{1}\right) \\
& =\beta \hat{\otimes}_{A}(\Phi \hat{\otimes} \mathrm{id})^{*}\left(\tau_{C^{*}(\Gamma)}\left([M]_{1}\right)\right) \\
& \stackrel{b}{=} \beta \hat{\otimes}_{A}(\Phi \hat{\otimes} \mathrm{id})_{*}\left(\tau_{C^{*}(\Gamma)}\left([M]_{1}\right)\right) \\
& =\beta \hat{\otimes}_{A}\left(\tau_{C^{*}(\Gamma)}\left([M]_{1}\right)\right) \hat{\otimes} \tau_{C l_{0, n}}(\phi) \\
& =\alpha\left([M]_{1}\right) \hat{\otimes}_{C^{*}(\Gamma) \hat{\otimes} C l_{0, n}} \tau_{C l_{0, n}}(\phi) \\
& =\Phi_{*}\left(\alpha\left([M]_{1}\right)\right)
\end{aligned}
$$

Equality a) is by Lemma 3.2 and equality b) is by [Bla98, 17.8.6]. 


\section{The $S^{1}$-bundle construction}

\subsection{Motivation: Bolotov and Dranishnikov's article}

The starting point for our investigation of the circle bundle construction is the preprint of an article by Bolotov and Dranishnikov ([BD14]), in which they examine the connection between positive scalar curvature and Gromov's ([Gro96]) notion of macroscopic dimension:

Definition 4.1.1. The macroscopic dimension of a metric space $X$, denoted $\operatorname{dim}_{m c}(X)$ is the smallest number $n \in \mathbb{Z}_{\geq 0} \cup\{\infty\}$ such that there exists an $n$-dimensional simplicial complex $K$ and a continuous map $f: X \rightarrow K$ such that

$$
\exists C \in \mathbb{R} \forall k \in K: \operatorname{diam}\left(f^{-1}(k)\right) \leq C
$$

Remark 4.1.2. The given requirement on the map $f$ is called "uniformly cobounded" by Dranishnikov. We note that $f$ is not required to be proper.

Example 4.1.3. The macroscopic dimension of a simplicial complex is always bounded above by the dimension of the complex. Gromov showed that for complete, uniformly contractible Riemannian manifolds equality holds: $\operatorname{dim}_{m c}(M)=\operatorname{dim}(M)$.

In particular, $\operatorname{dim}_{m c}\left(\mathbb{R}^{n}\right)=n$.

Example 4.1.4. For any $(n-2)$-dimensional manifold $M$,

$$
\operatorname{dim}\left(\widetilde{M \times S^{2}}\right)=n, \quad \text { but } \quad \operatorname{dim}_{m c}\left(\widetilde{M \times S^{2}}\right) \leq n-2
$$

This shows how macroscopic dimension can see the large scale features of a space. Note also that $M \times S^{2}$ is psc by Example 2.1.3.

Example 4.1.5. For $X=\left(\mathbb{T}^{n}\right)^{(k)}$, the $k$-skeleton of the torus, $k \geq 2$, the universal covering $\widetilde{X}$ is coarsely equivalent (in the sense of [HR00, 6.1.17]) to $\mathbb{R}^{n}$. But $\operatorname{dim}_{m c}(\tilde{X}) \leq k$.

This shows how macroscopic dimension can see the small scale features of a space.

Generalizing Example 4.1.4 Gromov conjectured:

Conjecture 4.1.6. Let $M$ be a closed psc manifold.

Then $\operatorname{dim}_{m c}(\widetilde{M}) \leq \operatorname{dim}(M)-2$. 
Both Bolotov and Dranishinikov investigated Gromov's notion of macroscopic dimension and made progress towards his conjecture. One sufficient condition for $\operatorname{dim}_{m c}(\widetilde{M}) \leq n-2$ is when the classifying map $u_{M}: M \rightarrow$ $\mathbf{B} \pi_{1}(M)$ of the universal covering $\widetilde{M}$ can be deformed into the $(n-2)$-skeleton $\mathbf{B} \pi_{1}(M)^{(n-2)}$. The question when such a deformation is possible can be attacked with obstruction theory. In [BD10] Bolotov and Dranishnikov showed that the obstruction can be seen in connective K-homology:

Theorem 4.1.7. Let $M$ be a closed Spin n-manifold with $n>3$, and suppose that a classifying map $u_{M}: M \rightarrow \mathbf{B} \pi_{1}(M)$ takes the ko-fundamental class to zero: $u_{M *}\left([M]_{k o}\right)=0 \in k o_{n}\left(\mathbf{B} \pi_{1}(M)\right)$.

Then $u_{M}$ can be homotoped into $\mathbf{B} \pi_{1}(M)^{(n-2)}$.

Because the image of $u_{M *}\left([M]_{k o}\right)$ under the map

$$
k o\left(\mathbf{B} \pi_{1}(M)\right) \longrightarrow K O\left(\mathbf{B} \pi_{1}(M)\right) \longrightarrow K O\left(C^{*}\left(\pi_{1}(M)\right)\right)
$$

is the Rosenberg index, which vanishes for psc manifolds, it follows:

Corollary 4.1.8. Conjecture 4.1.6 is true for Spin-manifolds whose fundamental groups satisfy the following conditions:

- The map $k o\left(\mathbf{B} \pi_{1}(M)\right) \rightarrow K O\left(\mathbf{B} \pi_{1}(M)\right)$ is injective.

- The map $K O\left(\mathbf{B} \pi_{1}(M)\right) \rightarrow K O\left(C^{*}\left(\pi_{1}(M)\right)\right)$ is injective.

Remark 4.1.9. The conditions on the fundamental group are the same as in Theorem 2.4.5. Bolotov and Dranishnikov call them the Rosenberg-Stolz conditions.

In the preprint BD14 Bolotov and Dranishnikov tried to show the following statement (Theorem 5.3):

Proposition 4.1.10. Let $M$ be a closed psc manifold of dimension $\geq 5$, such that $M$ is orientable but not Spin and $\pi_{1}(M)$ satisfies the Rosenberg-Stolz conditions. And let $u_{M}: M \rightarrow \mathbf{B} \pi_{1}(M)$ be a classifying map.

Then $u_{M *}([M])=0 \in H_{n}\left(\mathbf{B} \pi_{1}(M) ; \mathbb{Q}\right)$.

Their argument proceeds as follows: They find a Spin manifold $N^{\prime}$ with $\pi_{1}\left(N^{\prime}\right)=\pi_{1}(M)$ such that $u_{N^{\prime} *}([N]) \in H_{n}\left(\mathbf{B} \pi_{1}(M) ; \mathbb{Z}\right)$ is a non-zero multiple of $u_{M *}([M])$. By taking the connected sum with $\mathbb{C} P^{2} \times S^{n-4}$ and applying surgery they create a manifold $N$ with $\pi_{2}(N) \cong \mathbb{Z}$, such that $N$ is $\operatorname{Spin}^{c}$ but $\widetilde{N}$ is not Spin (compare Example 4.2.1). Because $M$ is psc, Theorem 2.1.7 implies that $N$ is psc.

Next, Bolotov and Dranishnikov construct an $S^{1}$ principal bundle $\pi_{L}: L \rightarrow$ $N$ such that $L$ is psc and Spin, $\pi_{2}(N)=0$ and $\pi_{L *}: \pi_{1}(L) \rightarrow \pi_{1}(N)$ is an isomorphism. From the last condition it follows that a classifying map $u_{L}: L \rightarrow$ $\mathbf{B} \pi_{1}(L)$ factors through $M$ and hence $u_{L}$ can be deformed into the $(\operatorname{dim}(L)-1)$ skeleton. Using obstruction theory they argue that $u_{L}$ can be deformed further 
into the $(\operatorname{dim}(L)-3)$-skeleton and by a rather long and technical argument involving spectral sequences they conclude that $u_{N^{\prime} *}\left(\left[N^{\prime}\right]\right)=u_{N *}([N])=0 \in$ $H_{n}\left(\mathbf{B} \pi_{1}(M) ; \mathbb{Z}\right)$.

The problem with their argument is that the $S^{1}$-bundle $L$ is the Spinboundary of the corresponding disk bundle (see Lemma 4.2.2). And because the bundle projection $\pi_{L}$ induces an isomorphism of fundamental groups, $u_{L *}\left([L]_{\text {Spin }}\right)=0 \in \Omega^{\operatorname{Spin}}\left(\mathbf{B} \pi_{1}(L)\right)$. This means that also $u_{L *}\left([L]_{K O}\right)=0 \in$ $K O\left(\mathbf{B} \pi_{1}(L)\right)$ and $\alpha\left([L]_{\text {Spin }}\right)=0 \in K O\left(C^{*}\left(\pi_{1}(L)\right)\right.$. Because $\pi_{1}(M)$ satisfies the Rosenberg-Stolz conditions, it follows that $L$ automatically has positive scalar curvature, even if the original manifold $M$ does not.

Actually, Bolotov and Dranishnikov need the fact that $L$ is psc only to show that $u_{L *}\left([L]_{k o}\right)=0 \in k o\left(\mathbf{B} \pi_{1}(L)\right)$. And this follows directly from $u_{L *}\left([L]_{\text {Spin }}\right)=0 \in \Omega^{\text {Spin }}\left(\mathbf{B} \pi_{1}(L)\right)$, again independent of whether or not $M$ is psc.

Therefore, the same argument could also be applied to $M=\mathbb{T}^{2 n} \# \mathbb{C} P^{n}$, the manifold of Example 4.2.1, for which the statement is false. Assuming that mathematics is consistent, the proof has to be wrong. In the final version of their article ([BD16]) Bolotov and Dranishnikov abandoned the argument altogether.

\subsection{The $S^{1}$-bundle construction}

Although the proof as a whole is flawed, Bolotov and Dranishnikov's argument in Proposition 4.1.10 includes the following interesting idea: If a manifold is not Spin but Spin $^{c}$, then we can form an $S^{1}$-principal bundle over the manifold that does allow a Spin-structure. The index obstruction can be computed for this bundle. Finally, a differential geometry argument shows that any obstruction against positive scalar curvature on the bundle is also an obstruction for the original manifold.

If this argument could provide non-vanishing obstructions against psc, it could possibly give new results about some manifolds where none of the classical methods works, including the following motivating example.

Example 4.2.1. Let $M=\mathbb{T}^{12} \# \mathbb{C} P^{6}$, the connected sum of a torus with a complex projective space. By computing the Stiefel-Whitney classes $w_{2}$ and $W_{3}$ one can see that $\mathbb{C} P^{6}$ is not Spin but Spin $^{c}$, and that the same is true for $M$ and $\widetilde{M}$. In fact, $M$ is perhaps the simplest example of a $\operatorname{Spin}^{c}$ manifold with non-trivial fundamental group and non-spin universal covering.

Because $M$ has a non-trivial fundamental group, Theorem 2.1.6 does not apply. Since the dimension is $>7$, the original theorem of Schoen and Yau does not work. Finally, $M$ is not Spin, hence index theory cannot be applied. In fact, it is not known (pending Schoen and Yau's latest preprint [SY17]), if $M$ admits a psc metric, although it is expected that it does not. 
We consider now a general closed $\operatorname{Spin}^{c}$ manifold $M$ such that $\widetilde{M}$ is not Spin. The bundle construction works as follows: The obstruction against the existence of a Spin-structure on the tangent bundle of an orientable manifold $M$ is the second Stiefel-Whitney class $w_{2} \in H^{2}\left(M ; \mathbb{Z}_{2}\right)$, and if $M$ admits a $\operatorname{Spin}^{c}$-structure, then the obstruction lifts to $H^{2}(M ; \mathbb{Z})$.

But $H^{2}(M ; \mathbb{Z})$ also classifies the $S^{1}$-principal bundles over $M$, since the Eilenberg-MacLane space $K(\mathbb{Z}, 2)=\mathbb{C} P^{\infty}$ is also the classifying space for $S^{1}$-bundles. Therefore, any lift $\bar{w}_{2} \in H^{2}(M ; \mathbb{Z})$ of $w_{2}$ defines an $S^{1}$-bundle $\pi_{L}: L \rightarrow M$.

Lemma 4.2.2. L is a Spin manifold.

Proof. $L$ is the boundary of the corresponding disk bundle $\bar{\pi}_{\bar{L}}: \bar{L} \rightarrow M$ sitting inside the complex line bundle $L^{\circ} \rightarrow M$. The tangent space of this disk bundle is the direct sum $T D=\bar{\pi}_{L}^{*}\left(T M \oplus L^{\circ}\right)$. But the Stiefel-Whitney class for $L^{\circ}$ is just the mod 2 reduction of the Euler class $\bar{w}_{2}$, that is $w_{2}(T M)$. And $w_{1}(T M)=w_{1}\left(L^{\circ}\right)=0$.

Therefore,

$$
\begin{aligned}
w_{2}(T L) & =\pi_{L}{ }^{*}\left(w_{2}\left(T M \oplus L^{\circ}\right)\right) \\
& =\pi_{L}{ }^{*}\left(w_{2}(T M)+w_{2}\left(L^{\circ}\right)\right) \\
& =\pi_{L}{ }^{*}\left(w_{2}(T M)+w_{2}(T M)\right) \\
& =\pi_{L}{ }^{*}(0)=0 \in H^{2}\left(\bar{L} ; \mathbb{Z}_{2}\right) .
\end{aligned}
$$

This means that the disk bundle has a Spin-structure, hence the $S^{1}$-bundle as its boundary also has a Spin-structure.

Finally, we have to see that if $M$ admits a metric of positive scalar curvature, then so does $L$. To do this, fix a metric $g_{M}$ of positive scalar curvature on $M$, an $S^{1}$-invariant metric $g_{S^{1}}$ on $S^{1}$, and use a partition of unity to obtain a principal $S^{1}$-connection on $L \rightarrow M$. Then by Vilms' theorem ([Bes08, 9.59]) there exists a unique metric $g_{L}$ on $L$ such that $\left(L, g_{L}\right) \rightarrow\left(M, g_{M}\right)$ is a Riemannian submersion, $g_{L}$ restricts to $g_{S^{1}}$ on the fibers and the horizontal distribution of the submersion is given by the principal connection.

If one multiplies the metric $g_{S^{1}}$ by some constant $t>0$, then the resulting metric $g_{L}$ changes with $t$. This is called the canonical variation. The scalar curvature of $L$ can be computed as follows ([Bes08, 9.70d]):

Theorem 4.2.3. Let $\kappa_{S^{1}}$ and $\kappa_{M}$ denote the scalar curvatures on the fiber and base space of a Riemannian submersion with totally geodesic fibers, and let $\kappa_{L}^{t}$ be the scalar curvature of the total space as a function of the scaling by $t$.

Then it holds:

$$
\kappa_{L}^{t}=\frac{1}{t} \cdot \kappa_{S^{1}}+\kappa_{M} \circ \pi_{L}-t \cdot|A|^{2},
$$

where $A$ is a tensor field on $M$ that is obtained from $g_{L}$ at $t=1$. 
Because $\kappa_{S^{1}}=0,|A|^{2}$ is bounded and $\kappa_{M}$ is bounded below by some $\epsilon>0$, it follows that $\kappa_{L}>0$ for $t>0$ sufficiently small. Geometrically, this means that the fiber is made small.

Remark 4.2.4. Theorem 4.2.3 could also be applied to fiber bundles with a different fiber than $S^{1}$ (if it can be made a totally geodesic Riemannian submersion). But it is important that $S^{1}$ admits a metric of vanishing scalar curvature, such that the summand $1 / t \cdot \kappa_{S^{1}}$ does not blow up.

And it is important that $S^{1}$ does not admit a metric of positive scalar curvature because otherwise, by starting with this metric and then making $t$ small, one would always get a psc metric on $L$, independent of $(M, g)$.

One obvious problem with the circle bundle construction is that $L$ is the Spin-boundary of the associated disk bundle and therefore represents zero in both $\Omega^{\text {Spin }}(p t)$ and $\Omega^{\text {Spin }}\left(\mathbf{B} \Gamma_{1}(M)\right)$. Since the index map factors through Spin bordism, it follows that the obstruction must be zero in $K O\left(C^{*}\left(\Gamma_{1}(M)\right)\right)$. In fact, if $\pi_{1}(L)=\pi_{1}(M)$, then Wiemeler showed ([Wie16, 1.5]) that $L$ admits a non- $S^{1}$-invariant psc metric.

Things are much less clear, however, when $\pi_{1}(L)$ differs from $\pi_{1}(M)$ and when one takes the index in $K O\left(C^{*}\left(\pi_{1}(L)\right)\right)$. In this case the disk bundle associated to $L$ does not provide a singular null-bordism of $L \rightarrow \mathbf{B} \pi_{1}(L)$. And in the case where the manifold $M$ is Spin there are simple examples, like the trivial $S^{1}$-bundle over a torus, where the Rosenberg index does not vanish for the bundle.

\subsection{The fundamental group of the $S^{1}$-bundle}

Let, as before, $M$ be a $\operatorname{Spin}^{c}$ manifold such that $\widetilde{M}$ is not Spin, and let $\pi_{L}: L \rightarrow M$ be an $S^{1}$-bundle such that $L$ is Spin. In this section we investigate the fundamental group $\pi_{1}(L)$, which depends on the $S^{1}$-bundle, and which plays a crucial role for the question when the index obstruction $\alpha\left([L]_{\text {Spin }}\right) \in$ $K O\left(C^{*}\left(\pi_{1}(L)\right)\right)$ vanishes.

Lemma 4.3.1. Let $M$ be a closed manifold with universal covering $p_{\widetilde{M}}: \widetilde{M} \rightarrow$ $M$, such that $M$ is Spin $^{c}$ but neither $M$ nor $\widetilde{M}$ are Spin. Let $L \rightarrow M$ be an $S^{1}$-bundle over $M$ such that $L$ is Spin, and let $x_{L} \in H^{2}(M ; \mathbb{Z})$ be the element that classifies $L$.

Then $\pi_{1}(L)$ is a central extension of $\pi_{1}(M)$ by a finite odd cyclic group $\mathbb{Z}_{n}$. Here $n$ is the largest integer $k$ such that $p_{\widetilde{M}}{ }^{*}\left(x_{L}\right)=0 \in H^{2}\left(\widetilde{M} ; \mathbb{Z}_{k}\right)$.

Proof. From the long exact sequence of the fibration $S^{1} \rightarrow L \rightarrow M$

$$
\ldots \longrightarrow \pi_{2}(M) \longrightarrow \pi_{1}\left(S^{1}\right) \longrightarrow \pi_{1}(L) \longrightarrow \pi_{1}(M) \longrightarrow \pi_{0}\left(S^{1}\right) \longrightarrow \ldots
$$

it follows that $\pi_{1}(L)$ is an extension of $\pi_{1}(M)$ by a quotient of $\pi_{1}\left(S^{1}\right)=\mathbb{Z}$. 
To see that this is a central extension, consider the restriction of the $S^{1}$ bundle to the 1-skeleton of $M$. This is the trivial $S^{1}$-bundle $S^{1} \times M^{(1)}$. Every element in $\pi_{1}(M)$ can be represented by a map $S^{1} \rightarrow M^{(1)}$ which can then be lifted to $\{p t\} \times M^{(1)} \subset S^{1} \times M^{(1)} \subset L$. All of these lifts commute in $\pi_{1}\left(S^{1} \times M^{(1)}\right)$ with the generator of $\pi_{1}\left(S^{1}\right)$, and this still holds true in $\pi_{1}(L)$, which is a quotient of $\pi_{1}\left(S^{1} \times M^{(1)}\right)$.

To find $n$ we have to consider the map $\partial_{2}: \pi_{2}(M) \rightarrow \pi_{1}\left(S^{1}\right) \cong \mathbb{Z}$ of the long exact sequence above. This map is precisely the evaluation map of the homomorphism

$$
p_{\widetilde{M}^{*}}^{*}(x) \in H^{2}(\widetilde{M} ; \mathbb{Z})=\operatorname{Hom}\left(H_{2}(\widetilde{M}, \mathbb{Z})\right)=\operatorname{Hom}\left(\pi_{2}(M), \mathbb{Z}\right) .
$$

Indeed, when $\phi: S^{2} \rightarrow M$ represents an element $[\phi] \in \pi_{2}(M)$, let $\pi_{L^{\prime}}: L^{\prime} \rightarrow$ $S^{2}$ be the pullback of $L \rightarrow M$ along $\phi . \quad L^{\prime}$ is the $S^{1}$-bundle classified by $\phi^{*}(x) \in H^{2}\left(S^{2} ; \mathbb{Z}\right)$.

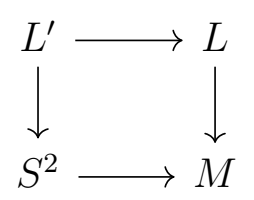

The identification $\pi_{1}\left(S^{1}\right) \cong \mathbb{Z}$ is made with the same isomorphism that was used for the correspondence of $S^{1}$-bundles with elements in $H^{2}(\cdot ; \mathbb{Z})$ (coming from a choice of orientation on the fibre of the canonical bundle over $\mathbb{C} P^{\infty}$ ), and the identification $\pi_{2}\left(S^{2}\right) \cong \mathbb{Z}$ is made with the same isomorphism that was used in the Hurewicz map above. Then $\partial_{2}([\phi])=\partial_{2}\left(\phi_{*}(1)\right)=\phi_{*}\left(\partial_{2}(1)\right)$, which is equal to the generator of $\pi_{1}\left(S^{1}\right)$ multiplied with the evaluation of $x$ on $[\phi]$.

But now $\operatorname{Im}\left(\partial_{2}: \pi_{2}(M) \rightarrow \pi_{1}\left(S^{1}\right)\right)$ is the same as $\operatorname{Im}\left(p_{\widetilde{M}}{ }^{*}(x): H_{2}(\widetilde{M} ; \mathbb{Z}) \rightarrow\right.$ $\mathbb{Z})$, which is the submodule of $\mathbb{Z}$ generated by the largest integer $k$ such that $p_{\widetilde{M}}{ }^{*}(x)=0 \in H^{2}\left(\widetilde{M} ; \mathbb{Z}_{k}\right)$.

Finally, the condition that $\widetilde{M}$ is not Spin means that $p_{\widetilde{M}}^{*}(x) \neq 0 \in$ $H^{2}\left(\widetilde{M} ; \mathbb{Z}_{2}\right)$, hence $p_{\widetilde{M}}{ }^{*}(x) \in \operatorname{Hom}\left(H_{2}(\widetilde{M} ; \mathbb{Z}), \mathbb{Z}\right)$ must assume an odd value on some homology element. But then the image of $\partial_{2}: \pi_{2}(M) \rightarrow \pi_{1}\left(S^{1}\right)$ is generated by an odd element.

This implies the following corollary about the set of Spin-structures on L:

Corollary 4.3.2. Let $\pi_{L}: L \rightarrow M$ be as above and let $\bar{L}$ be the disk bundle associated to $L$. The map

$$
\{\text { Spin-structures on } \bar{L}\} \rightarrow\{\text { Spin-structures on } L\}
$$

induced by taking the boundary of the disk bundle is a bijection.

Proof. The set of Spin-structures has a free transitive action of $H^{1}\left(\cdot ; \mathbb{Z}_{2}\right)$ and this action is compatible with taking boundaries. Therefore, it suffices 
to show that $\pi_{L}{ }^{*}: H^{1}\left(\bar{L} ; \mathbb{Z}_{2}\right) \rightarrow H^{1}\left(L ; \mathbb{Z}_{2}\right)$ is a bijection. But $H^{1}\left(\cdot ; \mathbb{Z}_{2}\right)=$ $\operatorname{Hom}\left(\pi_{1}(\cdot), \mathbb{Z}_{2}\right)$. And because $n$ is odd, the kernel of every homomorphism $\pi_{1}(L) \rightarrow \mathbb{Z}_{2}$ must contain the kernel of the extension $\mathbb{Z}_{n} \rightarrow \pi_{1}(L) \rightarrow \pi_{1}(M)$. Hence every homomorphism $\pi_{1}(L) \rightarrow \mathbb{Z}_{2}$ is the pullback of a (unique) homomorphism $\pi_{1}(\bar{L}) \cong \pi_{1}(M) \rightarrow \mathbb{Z}_{2}$ under the inclusion $L \subset \bar{L}$.

The isomorphism classes of central group extensions

$$
0 \longrightarrow \mathbb{Z}_{n} \longrightarrow \Gamma^{\prime} \longrightarrow \Gamma \longrightarrow 0
$$

correspond to elements in $H^{2}\left(\Gamma ; \mathbb{Z}_{n}\right)$. This is usually described in the language of 2-cocycles on the group $\Gamma$ (see [Bro82], IV.3). But the correspondence can also be seen in the picture of classifying spaces:

By [Mil56, 3.1] every model for the Eilenberg-MacLane space $K\left(\mathbb{Z}_{n}, 2\right)$ that has countably many cells can be seen as the base space of a classifying bundle $E \rightarrow K\left(\mathbb{Z}_{n}, 2\right)$ for principal bundles of a certain topological group $G$, which by the long exact sequence of homotopy groups has to be homotopy equivalent to $\mathrm{B} \mathbb{Z}_{n}$.

For $\phi: \mathbf{B} \Gamma \rightarrow K\left(\mathbb{Z}_{n}, 2\right)$ a representative of an element $[\phi] \in H^{2}\left(\mathbf{B} \Gamma ; \mathbb{Z}_{n}\right)$, the pullback $\phi^{*}(E)$ is aspherical. As in the proof of Lemma 4.3.1 one sees that the map $\pi_{1}\left(\phi^{*}(E)\right) \rightarrow \pi_{1}(\mathbf{B} \Gamma)$ induced by the projection is a central extension of $\Gamma$ by $\mathbb{Z}_{n}$.

Lemma 4.3.3. The central extension $\pi_{1}\left(\phi^{*}(E)\right) \rightarrow \pi_{1}(\mathbf{B} \Gamma)$ is given by the element $[\phi] \in H^{2}\left(\mathbf{B} \Gamma ; \mathbb{Z}_{n}\right)$.

Proof. For simplicity we choose a model for $\mathrm{B} \Gamma$ with one 1-cell for every group element and one 2 -cell for every relation $g_{1} g_{2}=g_{3}$. We also assume that $K\left(\mathbb{Z}_{n}, 2\right)$ has a base point $p t$, a trivial 1-skeleton and a single 2-cell, and that the map $\phi$ is cellular. Consider now the following diagram of maps between pairs of spaces, where the vertical maps are the bundle projections:

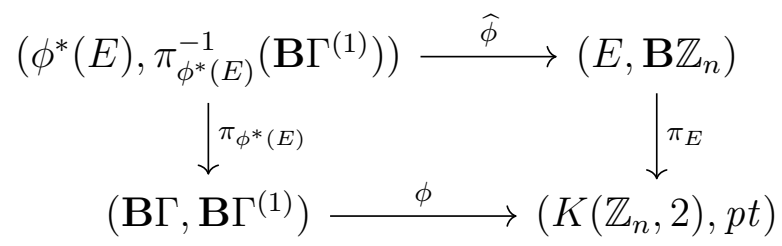

$\phi$ maps the 1-skeleton of $\mathbf{B} \Gamma$ to the base point of $K\left(\mathbb{Z}_{n}, 2\right)$, and this defines a trivialization of $\phi^{*}(E)$ over the 1-skeleton, which in turn defines a set-theoretical section $s: \Gamma \rightarrow \pi_{1}\left(\phi^{*}(E)\right)$. The 2-cocycle determined by this section is defined as $\xi\left(g_{1}, g_{2}\right)=\xi\left(g_{1}\right) \xi\left(g_{2}\right) \xi\left(g_{1} g_{2}\right)^{-1}$. To find its value on $\left(g_{1}, g_{2}\right)$ we consider the 2 -cell $D$ of the corresponding relation. There is a trivialization $\tau: \phi^{*}(E)_{\mid D} \rightarrow D \times \mathbf{B} \mathbb{Z}_{n}$. On $\partial D$ the difference between this trivialization and the first trivialization is given by a map $\eta: S^{1} \rightarrow \mathbf{B} \mathbb{Z}_{n}$, and $\xi\left(g_{1}, g_{2}\right)$ is given by the homotopy class $[\eta] \in \pi_{1}\left(\mathbf{B} \mathbb{Z}_{n}\right)=\mathbb{Z}_{n}$. 
To find this value let $\psi: D \rightarrow \phi^{*}(E)$ be the section determined by the trivialization $\tau$. $\xi\left(g_{1}, g_{2}\right)$ is given by $\partial_{2} \circ\left(\pi_{E}\right)_{*} \circ \widehat{\phi}_{*}([\psi]) \in \pi_{1}\left(\mathbf{B} \mathbb{Z}_{n}\right) \cong \mathbb{Z}_{n}$, where $[\psi] \in \pi_{2}(D, \partial D)$ is the relative homotopy class represented by $\psi$ and $\partial_{2}: \pi_{2}\left(K\left(\mathbb{Z}_{n}, 2\right)\right) \rightarrow \pi_{1}\left(\mathbf{B} \mathbb{Z}_{n}\right)$ is the boundary map of the long exact sequence.

But $\partial_{2}$ is an isomorphism. Furthermore, by commutativity of the above diagram,

$$
\left(\pi_{E}\right)_{*} \circ \widehat{\phi}_{*}([\psi])=\phi_{*} \circ\left(\pi_{\phi^{*}(E)}\right)_{*}([\psi])=\left(\phi_{\mid D}\right)_{*}([D, \partial D]) \in \pi_{2}\left(K\left(\mathbb{Z}_{n}, 2\right)\right) .
$$

This means that $\xi\left(g_{1}, g_{2}\right)$ captures the mapping degree of $\phi_{\mid D}$. After choosing compatible generators of $H^{2}\left(K\left(\mathbb{Z}_{n}, 2\right) ; \mathbb{Z}_{n}\right), \pi_{2}\left(K\left(\mathbb{Z}_{n}, 2\right)\right)$ and $\pi_{1}\left(\mathbf{B} \mathbb{Z}_{n}\right)$ the result is just the pullback of the 2 -cocycle representing $1 \in H^{2}\left(K\left(\mathbb{Z}_{n}, 2\right) ; \mathbb{Z}_{n}\right)$, evaluated on the cell $D$.

This finishes the proof.

We can now describe the central extension $\pi_{1}(L) \rightarrow \pi_{1}(M)$ in terms of the cohomology class $x_{L} \in H^{2}(M ; \mathbb{Z})$ that classifies $L$.

Lemma 4.3.4. Let $L \rightarrow M$ be an $S^{1}$-bundle such that $\mathbb{Z}_{n} \rightarrow \pi_{1}(L) \rightarrow \pi_{1}(M)$ is a central extension, and $u_{M}: M \rightarrow \mathbf{B} \pi_{1}(M)$ a classifying map. Let further $x_{L} \in H^{2}(M ; \mathbb{Z})$ be the element that classifies the bundle $L$ and let $\mu_{n}\left(x_{L}\right) \in$ $H^{2}\left(M ; \mathbb{Z}_{n}\right)$ be its image under the coefficient change.

Then $\mu_{n}\left(x_{L}\right)$ has a unique lift $y \in H^{2}\left(\mathbf{B} \pi_{1}(M) ; \mathbb{Z}_{n}\right)$ and this lift classifies the central extension $\pi_{1}(L) \rightarrow \pi_{1}(M)$.

Proof. First we lift $\mu_{n}\left(x_{L}\right)$ to $H^{2}\left(\mathbf{B} \pi_{1}(M) ; \mathbb{Z}_{n}\right)$. Note that $\mathbf{B} \pi_{1}(M)$ can be obtained from $M$ by attaching cells of dimension $\geq 3$. For the problem of extending $x_{L}$ to $\mathbf{B} \pi_{1}(M)$ only the attaching of 3-cells is relevant. We have to check that for every attaching map $\phi: S^{2} \rightarrow M$ of a 3-cell the pullback $\phi^{*}\left(\mu_{n}\left(x_{L}\right)\right)$ is zero.

But $\phi$ factors through $\widetilde{M}$, and Lemma 4.3.1 implies

$$
\phi^{*}\left(\mu_{n}\left(x_{L}\right)\right)=\widetilde{\phi}^{*}\left(p_{\widetilde{M}^{*}}^{*}\left(\mu_{n}\left(x_{L}\right)\right)\right)=0 .
$$

The lift is unique because one can assume that $\mathbf{B} \pi_{1}(M)$ and $M$ have identical 2-skeleta. Then every 2-cochain on $\mathbf{B} \pi_{1}(M)$ that restricts to a coboundary on $M$ is already a coboundary on $\mathbf{B} \pi_{1}(M)$.

Next we have to show that the lift $y \in H^{2}\left(\mathbf{B} \pi_{1}(M) ; \mathbb{Z}_{n}\right)$ classifies the central extension. Let $K(\mathbb{Z}, 2) \rightarrow K\left(\mathbb{Z}_{n}, 2\right)$ be the map representing the coefficient change $\mu_{n}: H^{*}(\cdot ; \mathbb{Z}) \rightarrow H^{*}\left(\cdot ; \mathbb{Z}_{n}\right)$. Because the total bundle $E(\mathbb{Z}, 2)$ is contractible, the map $E(\mathbb{Z}, 2) \rightarrow K(\mathbb{Z}, 2) \rightarrow K\left(\mathbb{Z}_{n}, 2\right)$ lifts to a bundle map $E(\mathbb{Z}, 2) \rightarrow E\left(\mathbb{Z}_{n}, 2\right)$. By naturality of the long exact sequence of homotopy groups this map induces a surjection on the fundamental groups of the fibers.

$\mu_{n}\left(x_{L}\right)$ defines via pullback a $\mathbf{B} \mathbb{Z}_{n}$-bundle $\widehat{L} \rightarrow M$ and a map of fiber bundles (not compatible with the group action) $L \rightarrow \widehat{L}$ that induces a surjection on the fundamental groups of the fibers. Then the induced map $\pi_{1}(L) \rightarrow \pi_{1}(\widehat{L})$ 
is a surjection. It is also an injection because the kernel is generated by $n$ times the generator of $\pi_{1}\left(S^{1}\right)$ and this element is already zero in $\pi_{1}(L)$ by assumption. Hence $\pi_{1}(L)=\pi_{1}(\widehat{L})$.

But since $\mu_{n}\left(x_{L}\right)$ is also the pullback of $y, \widehat{L}$ is isomorphic to the pullback of the $\mathbf{B} \mathbb{Z}_{n}$-bundle $\widehat{K} \rightarrow \mathbf{B} \pi_{1}(M)$ that corresponds to $y$. And the map $\widehat{L} \rightarrow \widehat{K}$ induces an isomorphism of fundamental groups. Then $\pi_{1}(L) \cong \pi_{1}(\widehat{L}) \cong \pi_{1}(\widehat{K})$, which is the central extension corresponding to $y$.

Finally, we address the question which central extensions of $\pi_{1}(M)$ can be realized as the fundamental group of a Spin $S^{1}$-bundle over the non-Spin manifold $M$.

Theorem 4.3.5. Let $M$ be a manifold such that $M$ is $\operatorname{Spin}^{c}$ but $\widetilde{M}$ is not Spin, and let $n$ be odd and $y \in H^{2}\left(\mathbf{B} \pi_{1}(M) ; \mathbb{Z}_{n}\right)$. Furthermore, assume that there exists a map $\phi: S^{2} \rightarrow M$ such that $\phi^{*}$ is surjective on $H^{2}(\cdot ; \mathbb{Z})$.

Then the following are equivalent:

1. There exists an $S^{1}$-bundle $L \rightarrow M$ such that $L$ is Spin and $\pi_{1}(L)$ is the central extension given by $y$.

2. $\delta_{B}\left(u_{M}{ }^{*}(y)\right)=0 \in H^{3}(M ; \mathbb{Z})$, where $\delta_{B}$ is the boundary map in the Bockstein sequence for the change of coefficients $\mu_{n}: \mathbb{Z} \rightarrow \mathbb{Z}_{n}$.

Proof. $1 \Rightarrow 2$ : Let $x_{L} \in H^{2}(M ; \mathbb{Z})$ classify $L$. Then

$$
\delta_{B}\left(u_{M}^{*}(y)\right)=\delta_{B}\left(\mu_{n}\left(x_{L}\right)\right)=0 .
$$

$2 \Rightarrow 1$ : Let $\delta_{B}\left(u_{M}{ }^{*}(y)\right)=0 \in H^{3}(M ; \mathbb{Z})$. Then by exactness of the Bockstein sequence there is some $x_{1} \in H^{2}(M ; \mathbb{Z})$ such that $u_{M}{ }^{*}(y)=\mu_{n}\left(x_{1}\right)$. $x_{1}$ is unique up to $n \cdot H^{2}(M ; \mathbb{Z})$.

Now $x_{1}$ defines an $S^{1}$-bundle $L_{1}$, but $L_{1}$ will not necessarily be $S p i n$. As before let $w_{2} \in H^{2}\left(M ; \mathbb{Z}_{2}\right)$ be the obstruction against $M$ having a Spin-structure and let $\bar{w}_{2} \in H^{2}(M ; \mathbb{Z})$ be any integral lift.

Let $x_{2}=x_{1}+n\left(\bar{w}_{2}-x_{1}\right)$. Then $\mu_{n}\left(x_{2}\right)=\mu_{n}\left(x_{1}\right)=u_{M}^{*}(y)$. Furthermore, $\mu_{2}\left(x_{2}\right)=w_{2}$. As in Lemma 4.2.2, it follows that the $S^{1}$-bundle $L_{2}$ corresponding to $x_{2}$ is Spin.

Finally, by Lemma 4.3.1, we have to ensure that $n$ is the largest integer such that $\mu_{n}\left(p_{\widetilde{M}^{*}}(x)\right)=0$. Let $\xi \in H^{2}(M ; \mathbb{Z})$ be such that $\phi^{*}(\xi)$ is a fixed generator $1 \in H^{2}\left(S^{2} ; \mathbb{Z}\right)$. And let $x_{3} \in H^{2}(M ; \mathbb{Z})$ be obtained from $x_{2}$ by adding a multiple of $2 n \xi$, such that $\phi^{*}\left(x_{3}\right)$ equals $n$ or $2 n$, depending on whether or not the pullback of $T M$ along $\phi$ is Spin.

Then the $S^{1}$-bundle corresponding to $x_{3}$ is also Spin and $\mu_{n}\left(x_{3}\right)=u_{M}{ }^{*}(y)$ as before. Furthermore, since $\phi$ factors through $\widetilde{\phi}: S^{2} \rightarrow \widetilde{M}, \mu_{k}\left(p_{\widetilde{M}}{ }^{*}\left(x_{3}\right)\right)$ can only be zero if $k$ divides $2 n$. But such a $k$ also has to be odd, whence $n$ is indeed the largest suitable integer. Now the $S^{1}$-bundle $L_{3}$ corresponding to $x_{3}$ is Spin, and its fundamental group is the extension of $\pi_{1}(M)$ by $\mathbb{Z}_{n}$ which is given by $y$. 
Remark 4.3.6. $\delta_{B}(y)=0 \in H^{3}(M ; \mathbb{Z})$ is a sufficient - but not necessary condition for $\delta_{B}\left(u_{M}{ }^{*}(y)\right)=0 \in H^{3}\left(\mathbf{B} \pi_{1}(M) ; \mathbb{Z}\right)$.

Finally, the structure of the fundamental group $\Gamma^{\prime}=\pi_{1}(L)$ leads to the first result about the index $\alpha\left([L]_{\text {Spin }}\right) \in K O\left(C^{*}\left(\Gamma^{\prime}\right)\right)$ :

Lemma 4.3.7. Let $\pi_{L}: L \rightarrow M$ as before, $\Gamma=\pi_{1}(M), \Gamma^{\prime}=\pi_{1}(L)$ and $u_{M}$, $u_{L}$ classifying maps.

Then $u_{L *}\left([L]_{\text {Spin }}\right) \in \Omega^{\text {Spin }}\left(\mathbf{B} \Gamma^{\prime}\right)$ has odd order.

In particular, it follows that $\alpha\left([L]_{\text {Spin }}\right) \in K O\left(C^{*}\left(\Gamma^{\prime}\right)\right)$ has odd order.

Proof. Let $\widehat{q}: \mathbf{B} \Gamma^{\prime} \rightarrow \mathbf{B} \Gamma$ be the map induced by the group homomorphism $q: \Gamma^{\prime} \rightarrow \Gamma$. The proof uses spectral sequences in order to show that the map $\widehat{q}_{*}: \Omega_{*}^{\text {Spin }}\left(\mathbf{B} \Gamma^{\prime}\right) \rightarrow \Omega_{*}^{\text {Spin }}(\mathbf{B} \Gamma)$ becomes an isomorphism after tensoring with $\mathbb{Z}\left[n^{-1}\right]$, i.e., after making $n$ invertible.

Because $\widehat{q}_{*} \circ u_{L *}\left([L]_{\text {Spin }}\right)=u_{M *} \circ \pi_{L *}\left([L]_{\text {Spin }}\right)=0 \in \Omega^{\text {Spin }}(\mathbf{B} \Gamma)$, this implies that $u_{L *}\left([L]_{\text {Spin }}\right)=0 \in \Omega^{\operatorname{Spin}}\left(\mathbf{B} \Gamma^{\prime}\right) \otimes \mathbb{Z}\left[n^{-1}\right]$ and therefore $u_{L *}\left([L]_{\text {Spin }}\right)$ must be an element of odd order.

First, note that $H_{*}\left(\mathbf{B} \mathbb{Z}_{n},\{p t\} ; \mathbb{Z}\right)$ consists purely of $n$-torsion. Hence the collapsing map $\mathbf{B} \mathbb{Z}_{n} \rightarrow\{p t\}$ induces an isomorphism $H_{*}\left(\mathbf{B} \mathbb{Z}_{n} ; \mathbb{Z}\right) \otimes \mathbb{Z}\left[n^{-1}\right] \cong$ $H_{*}(\{p t\} ; \mathbb{Z}) \otimes \mathbb{Z}\left[n^{-1}\right]$. Because $\mathbb{Z}\left[n^{-1}\right]$ is a flat module over $\mathbb{Z}$, it follows that also the induced map

$$
\operatorname{Tor}\left(H_{*-1}\left(\mathbf{B} \mathbb{Z}_{n} ; \mathbb{Z}\right), A\right) \otimes \mathbb{Z}\left[n^{-1}\right] \longrightarrow \operatorname{Tor}\left(H_{*-1}(\{p t\}), A\right) \otimes \mathbb{Z}\left[n^{-1}\right]
$$

is an isomorphism for all coefficient rings $A$. By applying the Five Lemma to the short exact sequence of the Universal Coefficient Theorem, using again flatness, one sees that the collapsing map induces an isomorphism $H_{*}\left(\mathbf{B} \mathbb{Z}_{n} ; A\right) \otimes$ $\mathbb{Z}\left[n^{-1}\right] \rightarrow H_{*}(\{p t\} ; A) \otimes \mathbb{Z}\left[n^{-1}\right]$.

The collapsing map induces a map between the spectral sequences $E$ and $F$ converging to $\Omega_{*}^{\text {Spin }}\left(\mathbf{B} \mathbb{Z}_{n}\right)$ and $\Omega_{*}^{\text {Spin }}(\{p t\})$, respectively. By the previous observation, the collapsing map induces an isomorphism $E^{2} \otimes \mathbb{Z}\left[n^{-1}\right] \rightarrow$ $F^{2} \otimes \mathbb{Z}\left[n^{-1}\right]$ between the second pages. Because $\mathbb{Z}\left[n^{-1}\right]$ is flat, the induced map on $E^{3} \otimes \mathbb{Z}\left[n^{-1}\right] \rightarrow F^{3} \otimes \mathbb{Z}\left[n^{-1}\right]$ is still an isomorphism, and the same is true for the following pages up to $E^{\infty}$. The Five Lemma then shows (again using flatness) that the collapsing map induces an isomorphism between the iterated extensions $\Omega_{*}^{\text {Spin }}\left(\mathbf{B} \mathbb{Z}_{n}\right) \otimes \mathbb{Z}\left[n^{-1}\right]$ and $\Omega_{*}^{\text {Spin }}(\{p t\}) \otimes \mathbb{Z}\left[n^{-1}\right]$. As before, it follows from the Universal Coefficient Theorem that the induced map $H_{*}\left(X ; \Omega_{*}^{\text {Spin }}\left(\mathbf{B} \mathbb{Z}_{n}\right)\right) \otimes \mathbb{Z}\left[n^{-1}\right] \rightarrow H_{*}\left(X ; \Omega_{*}^{\text {Spin }}(\{p t\})\right) \otimes \mathbb{Z}\left[n^{-1}\right]$ is an isomorphism for any space $X$.

Next, consider the generalized Atiyah-Hirzebruch spectral sequences $E^{\prime}$ and $F^{\prime}$ converging to the Spin-bordism groups of the spaces $\mathbf{B} \Gamma^{\prime}$ and $\mathbf{B} \Gamma$, both viewed as a fibration over $\mathbf{B} \Gamma$ with fiber $\mathbf{B} \mathbb{Z}_{n}$ and $\{p t\}$, respectively. In the case of $\mathbf{B} \Gamma^{\prime}$ the homotopy action of the fundamental group $\Gamma$ on the 
fiber $\mathbf{B} \mathbb{Z}_{n}$ is trivial because the fiber bundle, being classified by an element in $H^{2}\left(\mathbf{B} \Gamma ; \mathbb{Z}_{n}\right)$ is trivial over the 1-skeleton of $\mathbf{B} \Gamma$.

The projection map $\widehat{q}: \mathbf{B} \Gamma^{\prime} \rightarrow \mathbf{B} \Gamma$ is a map of fibrations and therefore induces a map between the spectral sequences $E^{\prime}$ and $F^{\prime}$. By the computation above the induced map is an isomorphism on $E^{\prime 2} \otimes \mathbb{Z}\left[n^{-1}\right] \rightarrow F^{\prime 2} \otimes \mathbb{Z}\left[n^{-1}\right]$. Again it follows from flatness of $\mathbb{Z}\left[n^{-1}\right]$ that the induced map gives an isomorphism on $E^{\prime \infty} \otimes \mathbb{Z}\left[n^{-1}\right] \rightarrow F^{\prime \infty} \otimes \mathbb{Z}\left[n^{-1}\right]$ and finally on $\Omega_{*}^{\text {Spin }}\left(\mathbf{B} \Gamma^{\prime}\right) \otimes \mathbb{Z}\left[n^{-1}\right] \rightarrow$ $\Omega_{*}^{\text {Spin }}(\mathbf{B} \Gamma) \otimes \mathbb{Z}\left[n^{-1}\right]$.

\subsection{A natural transformation from $\Omega^{\operatorname{Spin}^{c}}$ to $\Omega^{\text {Spin }}$}

The $S^{1}$-bundle construction as described in Section 4.2 involves choices for the $S^{1}$-bundle and the Spin-structure on it. In this section we will try to make these choices canonical; the goal is to turn the bundle construction into a natural transformation from $\Omega^{\text {Spin }}{ }^{c}$ to $\Omega^{\text {Spin }}$.

First, we consider the choice of the $S^{1}$-bundle. When $M$ is given with a specific $\operatorname{Spin}^{c}$-structure $s^{c}: M \rightarrow \mathbf{B} \operatorname{Spin}^{c}(k)$, then associated to the $\operatorname{Spin}^{c}$ structure there is a 1-dimensional complex line bundle called the determinant bundle (see [Fri00, p. 52]). The $U(1)$-bundle inside the determinant bundle is classified by the pullback along $s^{c}$ of a generator $e_{k} \in H^{2}\left(\mathbf{B} \operatorname{Spin}^{c}(k) ; \mathbb{Z}\right)$ (the choice of the generator corresponds to the choice of orientation on $S^{1}$ ). Since $\left(s^{c}\right)^{*}\left(e_{k}\right)=w_{2}(T M) \bmod 2$, the corresponding $S^{1}$-bundle $\pi_{L}: L \rightarrow M$ allows a Spin-structure.

When we fix an orientation on the group $S^{1}$ acting on $L$, this determines an orientation on $L$. However, the Spin-structure on $L$ is not determined by the $S_{p i n}{ }^{c}$-structure on $M$. For example, when $M=S^{1}$ with a given orientation, there is only one $\operatorname{Spin}^{c}$-structure on $M$, but there are four Spin-structures on the total space of the induced bundle $L=\mathbb{T}^{2} \rightarrow M$. Motivated by Corollary 4.3.2 we want to restrict ourselves to Spin-structures that are the boundary of Spin-structures on the determinant bundle. But for $M=S^{1}$ that still leaves two options.

Before we consider this further, however, we must also deal with a second problem, which is that the bundle $L \rightarrow M$ is Spin-null-bordant. In particular, for any choice of Spin-structures on the determinant bundle the map $\Omega_{*}^{\operatorname{Spin}^{c}}(\cdot) \rightarrow \Omega_{*+1}^{\text {Spin }}(\cdot)$ given by $(M, f) \mapsto\left(L, f \circ \pi_{L}\right)$ is the zero map. This problem can be circumvented by mapping $\Omega_{*}^{\operatorname{Spin}^{c}}(\cdot)$ not into $\Omega_{*+1}^{\text {Spin }}(\cdot)$ but into $\Omega_{*+1}^{\text {Spin }}\left(\cdot \times \mathbf{B} \mathbb{Z}_{n}\right)$ for some fixed odd $n$. Here $\Omega_{*}^{\text {Spin }}\left(\cdot \times \mathbf{B} \mathbb{Z}_{n}\right)$ is a functor, mapping $f: X \rightarrow Y$ to $(f \times \mathrm{id})_{*}: \Omega^{\operatorname{Spin}}\left(X \times \mathbf{B} \mathbb{Z}_{n}\right) \rightarrow \Omega^{\text {Spin }}\left(Y \times \mathbf{B} \mathbb{Z}_{n}\right)$.

In order to implement this, we start by fixing once and for all an odd number $n \in \mathbb{N}, n>1$, and generators $e_{k} \in H^{2}\left(\mathbf{B} \operatorname{Spin}^{c} ; \mathbb{Z}\right)$. We do this in such a way that $e_{k+1}$ maps to $e_{k}$ under the map induced by the canonical inclusion $\gamma_{k}: \mathbf{B} \operatorname{Spin}^{c}(k) \rightarrow \mathbf{B} \operatorname{Spin}^{c}(k+1)$. 
Then for all $k \in \mathbb{N}$ the element $n \cdot e_{k} \in H^{2}\left(\mathbf{B} \operatorname{Spin}^{c}(k) ; \mathbb{Z}\right)$ classifies an $S^{1}$ bundle $K_{k} \rightarrow \mathbf{B} \operatorname{Spin}^{c}(k)$. It satisfies $\pi_{1}\left(K_{k}\right)=\mathbb{Z}_{n}$, and we fix a classifying map (up to homotopy) $u_{k}: K_{k} \rightarrow \mathbf{B} Z_{n}$. $K_{k}$ is isomorphic to the pullback bundle $\gamma_{k}^{*}\left(K_{k+1}\right)$ and we choose the maps $u_{k}$ in such a way that $u_{k}$ factors through $u_{k+1}$.

If now $\left(M, s^{c}, f\right)$ is a manifold with $\operatorname{Spin}^{c}$-structure $s^{c}: M \rightarrow \mathbf{B} \operatorname{Spin}^{c}(k)$ and a singular map $f: M \rightarrow X$, we let $\pi_{L}: L \rightarrow M$ be the pullback of $K_{k}$ along $s^{c}$ and we let $L \rightarrow X \times \mathbf{B} \mathbb{Z}_{n}$ be the map $f \circ \pi_{L} \times u_{k} \circ \widehat{s^{c}}$, where $\widehat{s^{c}}: L \rightarrow K_{k}$ is the map of the pullback. This construction is compatible with passing to stable Spin $^{c}$-structures.

The Spin-structure on $L$ has yet to be determined and we have yet to show that the construction is compatible with the bordism relation. But first we give two examples that show that the construction can produce non-trivial elements in $\Omega^{\operatorname{Spin}}\left(X \times \mathbf{B} \mathbb{Z}_{n}\right)$.

Example 4.4.1. If $f: M=p t \rightarrow X=p t$, then the above procedure yields

$$
\left[\widehat{\psi}:\left(S^{1}, s_{\text {bord }}\right) \rightarrow \mathbf{B} \mathbb{Z}_{n}\right] \in \Omega_{1}^{S p i n}\left(\mathbf{B} \mathbb{Z}_{n}\right)
$$

where $s_{\text {bord }}$ is the bordant Spin-structure and $\widehat{\psi}$ the inclusion of the 1-skeleton $\mathbf{B} \mathbb{Z}_{n}{ }^{(1)}$.

This is a non-zero element of $\Omega^{\text {Spin }}\left(\mathbf{B} \mathbb{Z}_{n}\right)$.

Example 4.4.2. If $f=i d: M=S^{1} \rightarrow X=S^{1}$, then the procedure yields

$$
\chi=\left[(\mathrm{id}, \widehat{\psi}):\left(S^{1} \times S^{1}, s\right) \rightarrow S^{1} \times \mathbf{B} \mathbb{Z}_{n}\right]
$$

where $s$ is the bordant Spin-structure on the first $S^{1}$ factor and not yet determined on the second factor.

The projection $S^{1} \times \mathbf{B} \mathbb{Z}_{n} \rightarrow \mathbf{B} \mathbb{Z}_{n}$ and the inclusion $\mathbf{B} \mathbb{Z}_{n} \rightarrow S^{1} \times \mathbf{B} \mathbb{Z}_{n}$ induce a splitting $\Omega_{2}^{S p i n}\left(S^{1} \times \mathbf{B} \mathbb{Z}_{n}\right)=\Omega_{2}^{S p i n}\left(\mathbf{B} \mathbb{Z}_{n}\right) \oplus \Omega_{2}^{S p i n}\left(\Sigma \mathbf{B} \mathbb{Z}_{n}\right)$. We show that for both possible choices of $s$ the bordism class $\chi$ is non-zero in the second summand, which means that the map $\Omega_{1}^{\text {Spin }^{c}}\left(S^{1}\right) \rightarrow \Omega_{2}^{\text {Spin }}\left(S^{1} \times \mathbf{B} \mathbb{Z}_{n}\right)$ given by our construction does not factor through $\Omega_{2}^{\text {Spin }}\left(\mathbf{B} \mathbb{Z}_{n}\right)$.

Indeed, the boundary map of the Mayer-Vietoris sequence induces an isomorphism $\partial: \Omega_{2}^{\text {Spin }}\left(\Sigma \mathbf{B} \mathbb{Z}_{n}\right) \cong \Omega_{1}^{\text {Spin }}\left(\mathbf{B} \mathbb{Z}_{n}\right)$, and to compute $\partial(\chi)$ we have to restrict (id, $\widehat{\psi}$ ) to the preimage of $\{p t\} \times \mathbf{B} \mathbb{Z}_{n} \subset S^{1} \times \mathbf{B} \mathbb{Z}_{n}$. This means that $\partial(\chi)=\left[\widehat{\psi}:\left(S^{1}, s_{\text {bord }}\right) \rightarrow \mathbf{B} \mathbb{Z}_{n}\right]$, which is non-zero by the previous example.

We have to check that it is possible to choose the Spin-structure on the $S^{1}$-bundles in a consistent way. This is a serious issue as the following related problem shows:

Problem 4.4.3. Let $n \in \mathbb{N}$ be fixed. For every $\operatorname{Spin}^{c}$ manifold $\left(M, s^{c}\right)$ choose on the $S^{1}$-bundle $L_{n}$, produced from $\left(M, s^{c}\right)$ by the above construction, a Spin- 
structure $s$ (and therefore a Spin orientation $\left[L_{n}\right]_{\text {Spin }} \in \Omega^{\text {Spin }}\left(L_{n}\right)$ ), such that the following condition is fulfilled:

For every $S^{1}$-bundle map

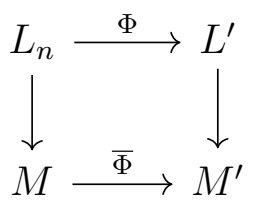

$\Phi_{*}\left(\left[L_{n}\right]_{\text {Spin }}\right)=0 \in \Omega^{\text {Spin }}\left(L^{\prime}\right)$ holds whenever $\bar{\Phi}_{*}\left([M]_{\text {Spin }^{c}}\right)=0 \in \Omega^{\operatorname{Spin}^{c}}\left(M^{\prime}\right)$.

Theorem 4.4.4. There is no solution to this problem.

Proof. Consider the case $M=\mathbb{T}^{2}$ with the $\operatorname{Spin}^{c}$-structure corresponding to a trivial bundle $L_{n} \rightarrow M$. And consider three maps $\bar{\Phi}_{1,0}, \bar{\Phi}_{0,1}$ and $\bar{\Phi}_{1,1}$ from $M$ to $S^{1}$. The first map is the projection onto the first $S^{1}$ factor of $M$, the second the projection onto the second factor, and the third is the product of these two, using the group structure of $S^{1}$. Let $\Phi_{., .}=\bar{\Phi}_{.,} \times i d: L_{n} \rightarrow S^{1} \times S^{1}$ be the corresponding maps between the total spaces of the bundles.

In all three cases the induced map on $\Omega^{\text {Spin }^{c}}$ sends the class $[M]_{\text {Spin }^{c}}$ to $0 \in \Omega_{2}^{\operatorname{Spin}^{c}}\left(S^{1}\right)=\Omega_{2}^{\text {Spin }^{c}}(p t) \oplus \Omega_{1}^{\text {Spinc }^{c}}(p t)$. The image of $[M]_{\text {Spin }^{c}}$ in $\Omega_{2}^{\text {Spin }^{c}}(p t)$ is zero because $M$ is null-bordant and $\Omega_{1}^{\text {Spin}^{c}}(p t)=0$.

Now let $s^{\prime}$ be any chosen Spin-structure on $L_{n}=\mathbb{T}^{2} \times S^{1}$ and $\left[L_{n}\right]_{\text {Spin }}$ the corresponding Spin-orientation. The fixed orientation on the $S^{1}$-fiber determines a trivialization of the normal bundle of $M \times p t \subset L_{n}$. Let $s^{\prime}$ be the induced Spin-structure on $M$ and $[M]_{\text {Spin }}$ the corresponding orientation class. Consider $\left(\Phi_{., .}\right)_{*}\left(\left[L_{n}\right]_{\text {Spin }}\right) \in \Omega_{3}^{\text {Spin }}\left(S^{1} \times S^{1}\right) \cong \Omega_{3}^{\text {Spin }}\left(S^{1}\right) \oplus \Omega_{2}^{\text {Spin }}\left(S^{1}\right)$, where the direct sum decomposition is made along the $S^{1}$-factor that is the fiber of the bundle $S^{1} \times S^{1} \rightarrow S^{1}$. The image of $\left[L_{n}\right]_{\text {Spin }}$ in the second

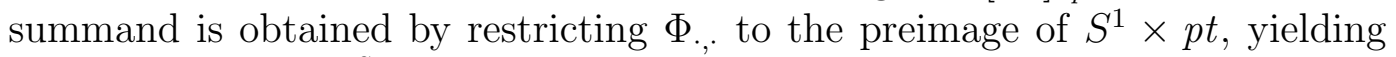
$\left(\bar{\Phi}_{.,}\right)_{*}\left([M]_{\text {Spin }}\right) \in \Omega_{2}^{\text {Spin }}\left(S^{1}\right)$.

After a second decomposition $\Omega_{2}^{\text {Spin }}\left(S^{1}\right)=\Omega_{2}^{\text {Spin }}(p t) \oplus \Omega_{1}^{\text {Spin }}(p t)$ the image of $[M]_{\text {Spin }}$ in the summand $\Omega_{1}^{\text {Spin }}(p t)$ is again obtained by restricting $\Phi_{\text {., }}$ to the preimage of some point, which is a circle with either the bording or the non-bording Spin-structure. The problem demands that the image of $\left[L_{n}\right]_{\text {Spin }}$ has to be zero for all three maps. In particular, it has to be zero in the summand $\Omega_{1}^{\text {Spin }}(p t)$, which means that for all three maps $\bar{\Phi}_{., .}$the restriction to the preimage $\left(\bar{\Phi}_{.,}\right)^{-1}(p t) \subset(M, s)$ must be the circle with the bordant Spin-structure.

Since $M$ is parallelizable, the bundle $P_{S O}(M)$ of oriented orthonormal frames in $T M$ is trivializable. A trivialization $\xi: P_{S O}(M) \rightarrow M \times S O(2)$ determines a Spin-structure $P_{\text {Spin }}(M)=M \times \operatorname{Spin} \rightarrow M \times S O \cong P_{S O}(M)$, and by retrivializing with a suitable map $M \rightarrow S O(2)$ we can achieve that the determined Spin-structure is $s$. When $S$ is any of the three preimage circles, we choose an orientation of the normal bundle $\nu(S)$ of $S \subset \mathbb{T}^{2}$. Then the 
induced Spin-structure on $S$ is determined by $\xi_{\mid S}$ which is a trivialization for the bundle of oriented orthonormal frames in $T S \oplus \nu(S)=T S \oplus(S \times \mathbb{R})$. We can compare $\xi_{\mid S}$ to the trivialization $\eta$ that comes from the decomposition $T S \oplus \nu(S)=(S \times \mathbb{R}) \oplus(S \times \mathbb{R})$ (where the orientation is chosen to match that of $\xi$ ) and determines the non-bordant ( $S^{1}$-equivariant) Spin-structure.

The difference between the trivializations $\xi_{\mid S}$ and $\eta$ is given by a map $\alpha(S): S \rightarrow S O(2)$. The two trivializations determine the same Spin-structure on $S$ iff the induced map $\alpha(S)_{*}: \pi_{1}(S) \rightarrow \pi_{1}(S O(2)) \rightarrow \mathbb{Z}_{2}$ is trivial, such that it can be lifted to $\alpha^{\prime}(S): S \rightarrow \operatorname{Spin}(2)$. Therefore, the induced Spin-structure on $S$ is the bordant one iff $\alpha(S)_{*}(1) \neq 0 \in \mathbb{Z}_{2}$. Thinking of $S$ as an immersed circle, this property does not change under regular homotopies. But we can homotope the preimage circles $S_{1,0}, S_{0,1}, S_{1,1}$ such that $S_{1,1}$ follows the path of first $S_{0,1}$ and then $S_{1,0}$. This means that $\alpha\left(S_{1,1}\right)_{*}=\alpha\left(S_{0,1}\right)_{*}+\alpha\left(S_{1,0}\right)_{*}$. Hence at least one of the three maps has to be trivial and at least one of the three preimage circles is not Spin-null-bordant. This gives a contradiction.

Although Problem 4.4.3 has a negative answer, there is still a way out: In the counterexample of the above proof we were working with $\Omega^{\operatorname{Spin}}\left(L^{\prime}\right)$, but in the actual setting we only have $\Omega^{\operatorname{Spin}}\left(M^{\prime} \times \mathbf{B} \mathbb{Z}_{n}\right)$, losing some information.

Theorem 4.4.5. Let $\left(f \circ \pi_{L_{n}} \times \widehat{\psi}\right): L_{n} \rightarrow X \times \mathbf{B} \mathbb{Z}_{n}$ be constructed from a singular Spin ${ }^{c}$ manifold $\left(f:\left(M, s^{c}\right) \rightarrow X\right)$ as explained above.

Then the bordism class $\left(f \circ \pi_{L_{n}} \times \widehat{\psi}\right)_{*}\left(\left[L_{n}\right]_{\text {Spin }}\right) \in \Omega^{\text {Spin }}\left(X \times \mathbf{B} \mathbb{Z}_{n}\right)$ does not depend on the choice of the (bordant along the $S^{1}$-fiber) Spin-structure on $L_{n}$.

Proof. Let $\left[L_{n}\right]_{1}$ and $\left[L_{n}\right]_{2}$ be the Spin orientations corresponding to two different Spin-structures on $L_{n}$. Then $\left[L_{n}\right]_{1}-\left[L_{n}\right]_{2}$ and hence $\left(f \circ \pi_{L_{n}} \times \widehat{\psi}\right)_{*}\left(\left[L_{n}\right]_{1}\right)-$ $\left(f \circ \pi_{L_{n}} \times \widehat{\psi}\right)_{*}\left(\left[L_{n}\right]_{2}\right)$ lies in the kernel of the natural transformation $\eta: \Omega^{\text {Spin }} \rightarrow$ $\Omega^{S O}$. Furthermore, since both Spin-structures on $L_{n}$ are induced from a Spinstructure on the corresponding disk bundle, both $\left[L_{n}\right]_{1}$ and $\left[L_{n}\right]_{2}$ map to zero in $\Omega^{\operatorname{Spin}}(X)$, which means that $\left(f \circ \pi_{L_{n}} \times \widehat{\psi}\right)_{*}\left(\left[L_{n}\right]_{1}\right)-\left(f \circ \pi_{L_{n}} \times \widehat{\psi}\right)_{*}\left(\left[L_{n}\right]_{2}\right)$ lies in the kernel of the map $p r_{*}: \Omega^{\operatorname{Spin}}\left(X \times \mathbf{B} \mathbb{Z}_{n}\right) \rightarrow \Omega^{\operatorname{Spin}}(X)$.

The proof of Lemma 4.3.7 also works in this situation to show that every element in $\operatorname{Ker}\left(p r_{*}\right)$ has odd order. Similarly, we now show that every element in the kernel of $\eta$ has order $2^{m}$ for some $m \in \mathbb{N}$. Both results together imply the claim.

To see that every element of $\operatorname{Ker}(\eta)$ has order $2^{m}$, we note that the map

$$
\eta(p t): \Omega^{S p i n}(p t) \rightarrow \Omega^{S O}(p t)
$$

becomes an isomorphism after inverting 2 (see [LM89, II.2]). For a general space $X$ we use the Atiyah-Hirzebruch spectral sequences

$$
E_{j, k}^{2}=H_{j}\left(X ; \Omega_{k}^{S p i n}(p t)\right) \Rightarrow \Omega^{S p i n}(X), \quad E_{j, k}^{\prime 2}=H_{j}\left(X ; \Omega_{k}^{S O}(p t)\right) \Rightarrow \Omega^{S O}(X) .
$$

The natural transformation $\eta$ induces a map between these spectral sequences. This map is an isomorphism on $E^{2} \otimes \mathbb{Z}\left[2^{-1}\right] \rightarrow E^{\prime 2} \otimes \mathbb{Z}\left[2^{-1}\right]$. Since 
$\mathbb{Z}\left[2^{-1}\right]$ is a flat module over $\mathbb{Z}$, it is still an isomorphism on $E^{3} \otimes \mathbb{Z}\left[2^{-1}\right] \rightarrow$ $E^{\prime 3} \otimes \mathbb{Z}\left[2^{-1}\right]$ and on the following pages up to $E^{\infty}$. The Five Lemma then shows (again using flatness) that $\eta$ is an isomorphism even between the iterated extensions $\Omega^{\operatorname{Spin}}(X) \otimes \mathbb{Z}\left[2^{-1}\right] \rightarrow \Omega^{S O}(X) \otimes \mathbb{Z}\left[2^{-1}\right]$.

This result means that the choice of the Spin-structure on the $S^{1}$-bundle in our construction is irrelevant. For every topological space $X$ we now define the map $T(X)$ from the set of isomorphism classes of singular Spin $^{c}$-manifolds in $X$ to the bordism group $\Omega^{\operatorname{Spin}}\left(X \times \mathbf{B} \mathbb{Z}_{n}\right)$ by sending $\left(M, s^{c}, f\right)$ to the bordism class $\left[L_{n}, s, f \circ \pi_{L_{n}} \times u_{K_{k}} \circ \widehat{s^{c}}\right] \in \Omega^{\operatorname{Spin}}\left(X \times \mathbf{B} \mathbb{Z}_{n}\right)$, where $\pi_{L_{n}}: L_{n} \rightarrow M$ is the bundle constructed above, $f \circ \pi_{L_{n}} \times u_{K_{k}} \circ \widehat{s}^{c}$ the map constructed above, and $s$ is any Spin-structure on $L$.

If $\left(M, s_{M}^{c}, f\right)$ is the boundary of a singular $\operatorname{Spin}^{c}$-manifold $\left(W, s_{W}^{c}, F\right)$, then $T\left(\left(M, s^{c}, f\right)\right)$ is the oriented boundary of the Spin-manifold $T\left(\left(W, s_{W}^{c}, F\right)\right)$, and we can rechoose the Spin-structure on $T\left(\left(M, s_{M}^{c}, f\right)\right)$ such that it is even the Spin-boundary. This means that $T(X)$ sends bordant manifolds to zero and thus defines a map

$$
\Omega_{*}^{\operatorname{Spin}^{c}}(X) \rightarrow \Omega_{*+1}^{\text {Spin }}\left(X \times \mathbf{B} \mathbb{Z}_{n}\right) .
$$

This map is natural because the singular map $f: M \rightarrow X$ is not used in the construction of the $S^{1}$-bundle, and it is compatible with the group addition $\left[M_{1}, s_{1}^{c}, f_{1}\right]+\left[M_{2}, s_{2}^{c}, f_{2}\right]=\left[M_{1} \sqcup M_{2}, s_{1}^{c} \sqcup s_{2}^{c}, f_{1} \sqcup f_{2}\right]$.

It also respects the boundary map of the Mayer-Vietoris exact sequence. Indeed, a Mayer-Vietoris decomposition $X=A \cup B$ induces a decomposition $X \times \mathbf{B} \mathbb{Z}_{n}=\left(A \times \mathbf{B} \mathbb{Z}_{n}\right) \cup\left(B \times \mathbf{B} \mathbb{Z}_{n}\right)$. If $\left(M, s^{c}, f\right)$ is a singular $\operatorname{Spin}^{c}$-manifold on $X$ and $N \subset M$ a codimension-1 submanifold such that $f(N) \subset A \cap B$, then $\left(M^{\prime}, f^{\prime}\right)=T\left(\left(M, s^{c}, f\right)\right)$ is a singular Spin-manifold on $X \times \mathbf{B} \mathbb{Z}_{n}$ and $\left(N^{\prime}, f_{\mid N^{\prime}}^{\prime}\right)=T\left(\left(N, s_{\mid N}^{c}, f_{\mid N}\right)\right)$ is a codimension-1 submanifold inside it with $f^{\prime}(N) \subset\left(A \times \mathbf{B} \mathbb{Z}_{n}\right) \cap\left(B \times \mathbf{B} \mathbb{Z}_{n}\right)$. The Mayer-Vietoris boundary map sends $\left[M, s^{c}, f\right]$ to $\left[N, s_{\mid N}^{c}, f_{\mid N}\right]$ and $\left[M^{\prime}, f^{\prime}\right]$ to $\left[N^{\prime}, f_{\mid N^{\prime}}^{\prime}\right]$, and thus commutes with the natural transformation induced by $T$.

In summary we have shown:

Theorem 4.4.6. For every odd number $n \in \mathbb{N}, n>1$, there is a non-trivial natural transformation of homology theories $\Omega_{*}^{\text {Spinc }}(\cdot) \rightarrow \Omega_{*+1}^{\text {Spin }}\left(\cdot \times \mathbf{B} \mathbb{Z}_{n}\right)$.

Remark 4.4.7. The construction always leads to an $S^{1}$-bundle that corresponds to an element in $n \cdot H^{2}(M ; \mathbb{Z})$, with $n$ odd. In the remaining sections we will again work in the most general setting where the $S^{1}$-bundle and the Spin-structure on it may be arbitrary.

\subsection{Rosenberg's example}

The result of Lemma 4.3.7 is somewhat disheartening - any obstruction that arises from the $S^{1}$-bundle construction would have to be quite subtle. The 
following example, due to Rosenberg ([Ros86]), deals with a very similar situation where such subtleties may occur. It shows that a non-psc manifold may have a finite, $n$-fold covering that is psc, even when $n$ is odd.

Consider the group $G=\mathbb{Z}^{2} \rtimes_{A} \mathbb{Z}$ where $\mathbb{Z}$ acts on $\mathbb{Z}^{2}$ via the matrix

$$
A=\left(\begin{array}{rr}
-1 & -1 \\
1 & 0
\end{array}\right) \in S L(2, \mathbb{Z}) .
$$

As $\mathbf{B} G$ one can take the 2-torus bundle over $S^{1}$ with twist given by $A$.

The homology groups $H_{*}(\mathbf{B} G ; \mathbb{Z})$ can be computed directly: $\mathbf{B} G$ can be decomposed into two parts by cutting $S^{1}$ along two points and cutting $\mathbf{B} G \rightarrow$ $S^{1}$ accordingly. The Mayer-Vietoris sequence is

$$
\begin{aligned}
& \longrightarrow H_{n}\left(\mathbb{T}^{2}\right) \oplus H_{n}\left(\mathbb{T}^{2}\right) \stackrel{\bar{A}}{\longrightarrow} H_{n}\left(\mathbb{T}^{2}\right) \oplus H_{n}\left(\mathbb{T}^{2}\right) \longrightarrow H_{n}(\mathbf{B} G) \\
& \hookrightarrow H_{n-1}\left(\mathbb{T}^{2}\right) \oplus H_{n-1}\left(\mathbb{T}^{2}\right) \stackrel{\bar{A}}{\longrightarrow} H_{n-1}\left(\mathbb{T}^{2}\right) \oplus H_{n-1}\left(\mathbb{T}^{2}\right) \longrightarrow H_{n-1}(\mathbf{B} G) \longrightarrow \\
& \text { with } \bar{A}=\left(\begin{array}{cc}
1 & 1 \\
1 & A_{*}
\end{array}\right) \text {. We may simplify this to } \\
& \begin{array}{r}
\left.\ldots \longrightarrow H_{n}\left(\mathbb{T}^{2}\right) \stackrel{A_{*}-1}{\longrightarrow} H_{n}\left(\mathbb{T}^{2}\right) \longrightarrow H_{n}(\mathbf{B} G)\right] \\
\hookrightarrow H_{n-1}\left(\mathbb{T}^{2}\right) \stackrel{A_{*}-1}{\longrightarrow} H_{n-1}\left(\mathbb{T}^{2}\right) \longrightarrow H_{n-1}(\mathbf{B} G) \longrightarrow \ldots
\end{array}
\end{aligned}
$$

a special case of the Wang sequence.

Here $A_{*}=\operatorname{det}(A)=1$ in degree $n=2$ and in degree $n=1$ the map $A_{*}-1$ is injective with a cokernel of order 3 . The only non-trivial extension problem is that for $H_{1}$, which is the abelianization of $G$, i.e., $\mathbb{Z} \oplus \mathbb{Z}_{3}$. We compute:

$H_{0}(\mathbf{B} G ; \mathbb{Z})=\mathbb{Z} ; \quad H_{1}(\mathbf{B} G ; \mathbb{Z})=\mathbb{Z} \oplus \mathbb{Z} 3 ; \quad H_{2}(\mathbf{B} G ; \mathbb{Z})=\mathbb{Z} ; \quad H_{3}(\mathbf{B} G ; \mathbb{Z})=\mathbb{Z}$

The $E^{2}$ page of the Atiyah-Hirzebruch spectral sequence that converges to $K O_{*}(\mathbf{B} G)$ looks like this:

\begin{tabular}{r|cccc}
$\vdots$ & $\vdots$ & $\vdots$ & $\vdots$ & $\vdots$ \\
5 & 0 & 0 & 0 & 0 \\
4 & $\mathbb{Z}$ & $\mathbb{Z} \oplus \mathbb{Z}_{3}$ & $\mathbb{Z}$ & $\mathbb{Z}$ \\
3 & 0 & 0 & 0 & 0 \\
2 & $\mathbb{Z}_{2}$ & $\mathbb{Z}_{2}$ & $\mathbb{Z}_{2}$ & $\mathbb{Z}_{2}$ \\
1 & $\mathbb{Z}_{2}$ & $\mathbb{Z}_{2}$ & $\mathbb{Z}_{2}$ & $\mathbb{Z}_{2}$ \\
0 & $\mathbb{Z}$ & $\mathbb{Z} \oplus \mathbb{Z}_{3}$ & $\mathbb{Z}$ & $\mathbb{Z}$ \\
\hline-1 & 0 & 0 & 0 & 0 \\
$\vdots$ & $\vdots$ & $\vdots$ & $\vdots$ & $\vdots$
\end{tabular}


The $E^{2}$ page of the Atiyah-Hirzebruch spectral sequence that converges to $\Omega_{*}^{\text {Spin }}(\mathbf{B} G)$ looks the same in the region that is shown here because the first few coefficients of the two homology theories coincide. Moreover, the natural transformation $\Omega_{n}^{\text {Spin }}(X) \rightarrow K O_{n}(X)$ is a bijection for $X=p t$ and $-3 \leq n \leq 7$ (In [Mil63] Milnor gives generators for $\Omega_{n}^{\text {Spin }}(p t), n<8$, and by [LM89, Section II.7] these map bijectively onto the generators of $K O(p t))$.

The entry $E_{1,4}^{2}=\mathbb{Z} \oplus \mathbb{Z}_{3}$ survives to the $E^{\infty}$ page and therefore is a subgroup of $\Omega_{5}^{\text {Spin }}(\mathbf{B} G)$ :

$$
0 \longrightarrow \mathbb{Z}_{0} \oplus \mathbb{Z}_{3} \longrightarrow \Omega_{5}^{\text {Spin }}(\mathbf{B} G) \longrightarrow \mathbb{Z}_{2} \longrightarrow 0
$$

In $E_{1,4}^{2}=H_{1}\left(\mathbf{B} G ; \Omega_{4}^{\text {Spin }}(p t)\right)$ there is an element of order 3 , which can be represented by a singular Spin manifold $[f: M \rightarrow \mathbf{B} G]$. By performing 0- and 1 - surgery on $M$, we can arrange for $f$ to induce an isomorphism of fundamental groups without changing the bordism class $[M, f] \in \Omega^{\operatorname{Spin}}(\mathbf{B} G)$.

Therefore, $f_{*}\left([M]_{K O}\right) \in K O_{5}(\mathbf{B} G)$ has order 3 . Since the group $G$ is torsion-free and solvable, it follows ([Ros86], 1.5) that the assembly map $K O_{*}(\mathbf{B} G) \rightarrow K O^{*}\left(C_{r}^{*}(G)\right)$ is an isomorphism. Hence the Rosenberg index does not vanish for $M$ and $M$ is not psc.

Because $A^{3}=1, \mathbb{Z}^{3}$ embeds into $G$ as a subgroup of index 3. Thus $p_{\mathbb{T}^{3}}: \mathbb{T}^{3} \rightarrow \mathbf{B} G$ is a threefold covering. Let $M^{\prime}$ be the pullback of this covering:

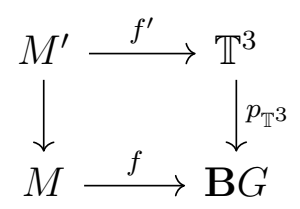

Then $\left[M^{\prime}, f^{\prime}\right]=p_{\mathbb{T}^{3}} !([M, f]) \in \Omega^{\operatorname{Spin}}\left(\mathbb{T}^{3}\right)$ is the image under the transfer homomorphism and must also have order three. But $\Omega^{\text {Spin }}\left(\mathbb{T}^{3}\right)$ does not contain elements of odd torsion, so $\left[M^{\prime}, f^{\prime}\right]=0 \in \Omega^{\operatorname{Spin}}\left(\mathbb{T}^{3}\right)$. Because $f^{\prime}$ is a classifying map for the universal covering of $M^{\prime}$, it follows that $\alpha\left(\left[M^{\prime}\right]_{\text {Spin }}\right)=0$. By Theorem 2.4.5 it follows further that $M^{\prime}$ admits a psc metric.

\subsection{The operator algebra side}

As before let $L \rightarrow M$ be a Spin circle-bundle over a $S_{p i n}{ }^{c}$ manifold with $\widetilde{M}$ non-Spin, and let $\Gamma^{\prime} \rightarrow \Gamma$ be the induced central extension of fundamental groups. In Lemma 4.3.7 it was shown that the index $\alpha\left([L]_{\text {Spin }}\right)$ must always have odd torsion.

In this section we look at the algebra $C^{*}\left(\Gamma^{\prime}\right)$ in order to determine when $K O\left(C^{*}\left(\Gamma^{\prime}\right)\right)$ can contain odd torsion and when it cannot. In this section we take $C^{*}\left(\Gamma^{\prime}\right)$ to be the reduced group $C^{*}$-algebra. We think of it as a Real 
$C^{*}$-algebra, that is, as a complex $C^{*}$-algebra with a given involution $\tau$ (see [HR00, Appendix B]).

Because $\mathbb{Z}_{n}$ is a central subgroup of $\Gamma^{\prime}$, there is a (non-real) homomorphism

$$
\zeta: C\left(\left\{e^{k / n \cdot 2 \pi i}\right\}_{0 \leq k<n}\right)=C_{r}^{*}\left(\mathbb{Z}_{n}\right) \rightarrow Z\left(C_{r}^{*}\left(\Gamma^{\prime}\right)\right)
$$

from the algebra of continuous $\mathbb{C}$-valued functions on the spectrum $\sigma\left(\mathbb{C}\left[\mathbb{Z}_{n}\right]\right) \subset$ $\mathbb{C}$ into the center of $C_{r}^{*}\left(\Gamma^{\prime}\right)$. In particular, there exist in $C_{r}^{*}\left(\Gamma^{\prime}\right) n$ central projections $p_{j}=1 / n \cdot \sum_{k \in \mathbb{Z}_{n}} e^{k j / n \cdot 2 \pi i} \delta_{k}$ with the property that $\sum_{j \in Z_{n}} p_{j}=1$. Therefore, $C_{r}^{*}\left(\Gamma^{\prime}\right)$ splits as a complex $C^{*}$-algebra into

$$
C_{r}^{*}\left(\Gamma^{\prime}\right)=\bigoplus_{j \in \mathbb{Z}_{n}} p_{j} C_{r}^{*}\left(\Gamma^{\prime}\right)
$$

The involution $\tau$ maps $p_{j}$ to $p_{-j}$. Hence it maps $p_{j} C_{r}^{*}\left(\Gamma^{\prime}\right)$ bijectively onto $p_{-j} C_{r}^{*}\left(\Gamma^{\prime}\right)$. As a Real $C^{*}$-algebra $C_{r}^{*}\left(\Gamma^{\prime}\right)$ splits into

$$
C_{r}^{*}\left(\Gamma^{\prime}\right)=p_{0} C_{r}^{*}\left(\Gamma^{\prime}\right) \oplus \bigoplus_{0<j<n / 2}\left(p_{j} C_{r}^{*}\left(\Gamma^{\prime}\right) \oplus p_{-j} C_{r}^{*}\left(\Gamma^{\prime}\right)\right)
$$

In K-theory this becomes

$$
K O\left(C_{r}^{*}\left(\Gamma^{\prime}\right)\right)=K O\left(p_{0} C_{r}^{*}\left(\Gamma^{\prime}\right)\right) \oplus \bigoplus_{0<j<n / 2} K O\left(p_{j} C_{r}^{*}\left(\Gamma^{\prime}\right) \oplus p_{-j} C_{r}^{*}\left(\Gamma^{\prime}\right)\right) .
$$

The map $q_{*}: C_{r}^{*}\left(\Gamma^{\prime}\right) \rightarrow C_{r}^{*}(\Gamma)$ induced by $q: \Gamma^{\prime} \rightarrow \Gamma$ (the induced map exists, because the kernel of the group extension is finite) is surjective because $q$ is surjective (and $C^{*}$-homomorphisms have closed images). It sends $p_{0}$ to $1 \in$ $C_{r}^{*}(\Gamma)$ and $p_{j}$ to 0 for $j \neq 0$. Restricted to $p_{0} C_{r}^{*}\left(\Gamma^{\prime}\right)$ the map is injective because it is injective on the dense subspace $p_{0} \mathbb{C}\left[\Gamma^{\prime}\right]$. Therefore, $p_{0} C_{r}^{*}\left(\Gamma^{\prime}\right) \cong C_{r}^{*}(\Gamma)$ as Real $C^{*}$-algebras and in the decomposition of Equation 4.1 the map $q_{*}$ is just the projection onto the first summand.

On the topological side, the push-forward $u_{L *}\left([L]_{K O}\right) \in K O\left(\mathbf{B} \Gamma^{\prime}\right)$ gets mapped to zero under the map $K O\left(\mathbf{B} \Gamma^{\prime}\right) \rightarrow K O(\mathbf{B} \Gamma)$. Since the assembly map is natural, the index must be zero in the summand $K O\left(C_{r}^{*}(\Gamma)\right)$. This only leaves the summands $K O\left(p_{j} C_{r}^{*}\left(\Gamma^{\prime}\right) \oplus p_{-j} C_{r}^{*}\left(\Gamma^{\prime}\right)\right)$. Because the involution exchanges $p_{j} C_{r}^{*}\left(\Gamma^{\prime}\right)$ and $p_{-j} C_{r}^{*}\left(\Gamma^{\prime}\right)$, these groups are isomorphic to the complex K-theory groups $K\left(p_{j} C_{r}^{*}\left(\Gamma^{\prime}\right)\right)$.

The complex $C^{*}$-algebra $p_{j} C_{r}^{*}\left(\Gamma^{\prime}\right)$ can be viewed as a twisted group $C^{*}$ algebra of the group $\Gamma$.

Definition 4.6.1. Let $G$ be a discrete group and $\omega: G \times G \rightarrow \mathbb{T}$ a 2-cocycle on $G$ (i.e., $\omega(s, t) \omega(r, s t)=\omega(r, s) \omega(r s, t), \omega(1, s)=\omega(s, 1)=1$ for all $r, s, t \in G)$.

The algebraic twisted group algebra $\mathbb{C}[G ; \omega]$ is defined as the vector space of finitely supported functions from $G$ to $\mathbb{C}$, with multiplication and involution given by

$$
\delta_{g} \cdot \delta_{h}=\omega(g, h) \delta_{g h}, \quad \delta_{g}^{*}=\omega\left(g, g^{-1}\right)^{-1} \delta_{g^{-1}}
$$


The left-regular $\omega$-representation of $\mathbb{C}[G ; \omega]$ on $\ell^{2}(G)$ is given by (the linear extension of)

$$
\delta_{g} \cdot \delta_{h}=\omega(g, h) \delta_{g h}
$$

The reduced twisted group algebra $C_{r}^{*}(G ; \omega)$ is the completion of $\mathbb{C}[\Gamma ; \omega]$ with respect to the left-regular $\omega$-representation.

If two cocycles $\omega_{1}, \omega_{2}$ are cohomologous, the resulting $C^{*}$-algebras are isomorphic.

The following is a special case of a classical result (see, e.g., EL69, Section $3])$.

Lemma 4.6.2. Let $\Gamma^{\prime} \rightarrow \Gamma$ be a central extension of a discrete group by a finite cyclic group $\mathbb{Z}_{n}$, and let $p_{j}$ be the central projections in $C_{r}^{*}\left(\Gamma^{\prime}\right)$ as above.

Then $p_{j} C_{r}^{*}\left(\Gamma^{\prime}\right)$ is (isomorphic to) a twisted group $C^{*}$-algebra $C_{r}^{*}\left(\Gamma ; \omega_{j}\right)$ with the twist $\omega_{j}$ determined by the cohomology class that classifies the central extension.

Proof. Let $s: \Gamma \rightarrow \Gamma^{\prime}$ be any (set-theoretic) split of the quotient map $q: \Gamma^{\prime} \rightarrow$ $\Gamma$, and let $\xi: \Gamma \times \Gamma \rightarrow \mathbb{Z}_{n}, \xi\left(\gamma_{1}, \gamma_{2}\right)=s\left(\gamma_{1}\right) s\left(\gamma_{2}\right)\left(s\left(\gamma_{1} \gamma_{2}\right)\right)^{-1}$ be the resulting cocycle, representing the extension $\Gamma^{\prime} \rightarrow \Gamma$ (see [Bro82, IV.3]). $s$ extends linearly to a (non-multiplicative) map between the group algebras $\mathbb{C}[\Gamma]$ and $\mathbb{C}\left[\Gamma^{\prime}\right]$. The (non-multiplicative) linear maps $\eta_{j}: \mathbb{C}[\Gamma] \rightarrow p_{j} \mathbb{C}\left[\Gamma^{\prime}\right]$ defined by $\delta_{\gamma} \mapsto p_{j} s(\gamma)$ are bijective. The multiplication transforms in the following way:

$$
\begin{aligned}
\eta_{j}\left(\delta_{\gamma_{1}}\right) \cdot \eta_{j}\left(\delta_{\gamma_{2}}\right) & =p_{j} \cdot s\left(\delta_{\gamma_{1}}\right) \cdot s\left(\delta_{\gamma_{2}}\right) \\
& =p_{j} \cdot \xi\left(\gamma_{1}, \gamma_{2}\right) \cdot s\left(\delta_{\gamma_{1} \gamma_{2}}\right) \\
& =e^{j \cdot \xi\left(\gamma_{1}, \gamma_{2}\right) \cdot 2 \pi i / j} \cdot p_{j} \cdot s\left(\delta_{\gamma_{1} \gamma_{2}}\right) \\
& =e^{j \cdot \xi\left(\gamma_{1}, \gamma_{2}\right) \cdot 2 \pi i / j} \cdot \eta_{j}\left(\delta_{\gamma_{1} \gamma_{2}}\right)
\end{aligned}
$$

Therefore, $p_{j} \mathbb{C}\left[\Gamma^{\prime}\right]$ can be identified with the twisted group algebra $\mathbb{C}\left[\Gamma ; \omega_{j}\right]$ where $\omega_{j} \in Z(\Gamma ; \mathbb{T})$ is the cocycle $j \cdot \xi$.

The $C^{*}$-algebra $p_{j} C_{r}^{*}\left(\Gamma^{\prime}\right)$ is the completion of $p_{j} \mathbb{C}\left[\Gamma^{\prime}\right]$ with respect to the left action on the Hilbert space $p_{j} \ell^{2}\left(\Gamma^{\prime}\right)$. Because $\eta_{j}$ is an isometry up to a factor of $\sqrt{n}$, it extends to an isomorphism of Hilbert spaces betweeen $\ell^{2}(\Gamma)$ and $p_{j} \ell^{2}\left(\Gamma^{\prime}\right)$. With this identification the action satisfies

$$
\eta_{j}\left(\delta_{\gamma_{1}}\right) \cdot \delta_{\gamma_{2}}=\omega_{j}\left(\gamma_{1}, \gamma_{2}\right) \cdot \delta_{\gamma_{1} \gamma_{2}}
$$

Therefore, the left-regular action $p_{j} \mathbb{C}\left[\Gamma^{\prime}\right] \curvearrowright p_{j} \ell^{2}\left(\Gamma^{\prime}\right)$ is equivalent to the left-regular $\omega_{j}$-representation of $\mathbb{C}\left[\Gamma ; \omega_{j}\right]$. Hence $p_{j} C_{r}^{*}\left(\Gamma^{\prime}\right) \cong C_{r}^{*}\left(\Gamma ; \omega_{j}\right)$.

For the K-theory of twisted group $C^{*}$-algebras there is the following result (proved in [ELPW10, Theorem 0.3] for locally compact groups).

Theorem 4.6.3. Let $\omega_{1}, \omega_{2} \in Z^{2}(\Gamma ; \mathbb{T})$ be homotopic cocycles and assume that $\Gamma$ satisfies the Baum-Connes conjecture with coefficients.

Then $K_{*}\left(C_{r}^{*}\left(\Gamma ; \omega_{1}\right)\right) \cong K_{*}\left(C_{r}^{*}\left(\Gamma ; \omega_{2}\right)\right)$. 
This theorem can be applied to the motivating Example 4.2.1.

Lemma 4.6.4. For $M=\mathbb{C} P^{2 k} \# \mathbb{T}^{4 k}$ the circle bundle construction never gives a non-vanishing obstruction.

Proof. By Lemma 4.3.7 $\alpha\left([L]_{\text {Spin }}\right)$ must be an element of odd order. Hence it suffices to show that $K O\left(C_{r}^{*}\left(\Gamma^{\prime}\right)\right) \cong K O\left(C_{r}^{*}(\Gamma)\right) \oplus \bigoplus K\left(C_{r}^{*}\left(\Gamma ; \omega_{j}\right)\right)$ does not contain odd torsion.

The group $\Gamma=\mathbb{Z}^{4 k}$ is amenable and hence ([HK01, Theorem 1.1]) satisfies the Baum-Connes conjecture with coefficients. Furthermore, we have the following commutative diagram, where all maps are changes of coefficients:

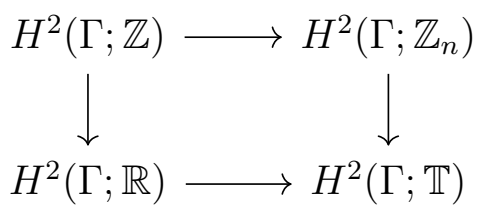

The twists $\omega_{j} \in H^{2}(\Gamma ; \mathbb{T})$ come from $H^{2}\left(\Gamma ; \mathbb{Z}_{n}\right)$. For $\Gamma=\mathbb{Z}^{4 k}$ the top horizontal map is surjective. Therefore, the $\omega_{j}$ can be lifted to $H^{2}(\Gamma ; \mathbb{Z})$ and hence to $H^{2}(\Gamma ; \mathbb{R})$. There, every cocycle is null-homotopic via the homotopy $\omega_{j}(t)=t \cdot \omega_{j}, t \in[0,1]$. Therefore, by the previous theorem,

$$
K O_{*}\left(C_{r}^{*}\left(\Gamma^{\prime}\right)\right) \cong K O_{*}\left(C_{r}^{*}(\Gamma)\right) \oplus \bigoplus K_{*}\left(C_{r}^{*}(\Gamma)\right) \cong K O^{*}\left(\mathbb{T}^{4 k}\right) \oplus \bigoplus K^{*}\left(\mathbb{T}^{4 k}\right),
$$

which does not contain odd torsion.

It is clear that this reasoning also works for many other examples of circle bundles. It does not apply if either $K O\left(C_{r}^{*}(\Gamma)\right)$ contains odd torsion, or the Bockstein map $H^{2}\left(\Gamma ; \mathbb{Z}_{n}\right) \rightarrow H^{3}(\Gamma ; \mathbb{Z})$ is non-zero, or $\Gamma$ does not satisfy the Baum-Connes conjecture with coefficients.

\subsection{Vanishing of the index}

As before let $\pi_{L}: L \rightarrow M$ be a Spin circle bundle over a closed $\operatorname{Spin}^{c}$ manifold with $\widetilde{M}$ non-Spin. And let $\Gamma=\pi_{1}(M), \Gamma^{\prime}=\pi_{1}(L)$ be the fundamental groups. $\Gamma^{\prime}$ is a central extension of $\Gamma$ by a finite cyclic group.

We will now show that the Rosenberg index of the bundle $L$ always vanishes. Therefore, no index-theoretic obstruction against positive scalar curvature can be obtained from the bundle construction. The proof requires equivariant bordism groups and equivariant K-homology groups, which we briefly recall.

\section{The equivariant viewpoint}

Definition 4.7.1. Let $M$ be a smooth manifold and $\Gamma \curvearrowright M$ a proper action of a discrete group. In this case there exists a $\Gamma$-invariant Riemannian metric 
on $M$ and hence the derivative of the action defines an action of $\Gamma$ on the $O(n)$-bundle $P_{O}$ of orthonormal frames in $T M$.

A $\Gamma$-Spin-structure on $T M$ consists of a principal Spin $(n)$-bundle $P_{\text {Spin }}$, an action $\Gamma \curvearrowright P_{\text {Spin }}$ and a $\Gamma$-equivariant bundle map $P_{\text {Spin }} \rightarrow P_{O}$ that restricts in each fiber to the canonical map $\operatorname{Spin}(n) \rightarrow O(n)$. Two Spin-structures $P_{\text {Spin }} \rightarrow P_{O}$ and $P_{\text {Spin }}^{\prime} \rightarrow P_{O}$ are equivalent if there is an equivariant bundle isomorphism $P_{S p i n} \rightarrow P_{S p i n}^{\prime}$ that is compatible with the maps to $P_{O}$.

As in the non-equivariant case we can pass to stable Spin-structures. If the $\Gamma$-manifold $M$ has a boundary, then we can stabilize $T \partial M$ by adding to it the normal bundle of $\partial M \subset M$, which is canonically identified with $\partial M \times \mathbb{R}$. Then the restriction of a $\Gamma$-Spin-structure on $T M$ to $\partial M$ defines a $\Gamma$-Spin-structure on $T \partial M \oplus(\partial M \times \mathbb{R})$.

Example 4.7.2. If $\widetilde{M}$ is the universal covering of the closed manifold $M$ and $s: P_{\text {Spin }} \rightarrow P_{O}$ is a Spin-structure on $M$, then the bundle of orthonormal frames on $T \widetilde{M}$ can be identified with the pullback $P_{O} \times_{M} \widetilde{M}$, and the Spin-structure $s$ defines a $\pi_{1}(M)$-Spin-structure on $\widetilde{M}$ given by $P_{\text {Spin }} \times_{M} \widetilde{M} \rightarrow P_{O} \times_{M} \widetilde{M}$.

In the other direction, if a $\pi_{1}(M)$-Spin-structure on $\widetilde{M}$ is given, then by quotienting out the $\pi_{1}(M)$-action one gets a Spin-structure on $M$.

The equivariant Spin-bordism groups are now defined analogously to Definition 2.3.1.

Definition 4.7.3. Let $X$ be a topological space and $\Gamma \curvearrowright X$ a proper action of a discrete group.

The equivariant Spin-bordism group $\Omega_{k}^{\Gamma}(X)$ is the abelian group of bordism classes of $\Gamma$-equivariant maps $(M, s) \rightarrow X$, where $M$ is a $\Gamma$-compact, proper $\Gamma$-manifold of dimension $k$, without boundary, and $s$ is a $\Gamma$-Spin-structure on $M$.

There is also an equivariant version of geometric K-homology that is equivalent to the analytic version. It is the equivariant analogue of Definition 2.3.3. See [BHS10] for a complete definition, the proof of equivalence in the complex case (the real case is analogous, see their Remark 4.1) and the relation to the Baum-Connes map. See also [BOOSW10] and [GMW].

Definition 4.7.4. Let $(X, A)$ be a pair of topological spaces and $\Gamma \curvearrowright(X, A)$ a proper action of a discrete group. Elements of $K O_{n}^{\Gamma}(X, A)$ are represented by quadruples $(M, s, E, f)$, where $M$ is a $\Gamma$-compact, proper $\Gamma$-manifold of dimension $n \bmod 8, s$ is a $\Gamma$-Spin-structure on $M, E \rightarrow M$ a real $\Gamma$-vector bundle and $f: M \rightarrow X$ a continuous $\Gamma$-equivariant map such that $f(\partial M) \subset A$. The equivalence relation is generated by:

1. Direct sum of vector bundles equals disjoint union

2. Equivariant bordism

3. Equivariant vector bundle modification 
As in the non-equivariant case there is the natural transformation that sends $[M, s, f] \in \Omega_{*}^{\Gamma}(X)$ to $[M, s, M \times \mathbb{R}, f] \in K O_{*}^{\Gamma}(X)$.

Furthermore, as expected for equivariant homology theories (see [Lüc02a]) there are induction structures on the equivariant Spin-bordism groups and the equivariant K-homology groups: If $X$ is a $\Gamma$-space and $\phi: \Gamma \rightarrow G$ a group homorphism with kernel acting freely on $X$, then there are functorial isomorphisms

$$
\Omega_{k}^{\Gamma}(X) \cong \Omega_{k}^{G}\left(X \times_{\Gamma} G\right) \quad \text { and } \quad K O_{k}^{\Gamma}(X) \cong K O_{k}^{G}\left(X \times_{\Gamma} G\right) .
$$

In the special case where $\pi_{1}(M)$ acts freely on the universal covering $\widetilde{M}$ of a closed Spin-manifold $(M, s)$ and $\phi: \pi_{1}(M) \rightarrow\{e\}$ is the trivial map this correspondence sends $[M, s, i d] \in \Omega_{n}^{\operatorname{Spin}}(M)$ to $[\widetilde{M}, \widetilde{s}, i d] \in \Omega_{n}^{\Gamma}(\widetilde{M})$ and $[M, s, M \times \mathbb{R}, i d] \in K O_{n}(M)$ to $[\widetilde{M}, \widetilde{s}, \widetilde{M} \times \mathbb{R}, i d] \in K O_{n}^{\Gamma}(\widetilde{M})$.

\section{The vanishing of the index}

As mentioned in Section 2.4, the Rosenberg index

$$
\alpha: \Omega^{\operatorname{Spin}}(M) \rightarrow K O\left(C^{*}\left(\pi_{1}(M)\right)\right)
$$

factors through the equivariant K-homology group $K O^{\Gamma}\left(\underline{\mathbf{E}} \pi_{1}(M)\right)$. Here $\underline{\mathbf{E}} \Gamma$ denotes the classifying space for proper $\Gamma$-actions (see [Lüc02b], 1.28). It is a proper $\Gamma$-CW-complex, determined up to $\Gamma$-equivariant homotopy by the requirement that for every $x \in \underline{\mathbf{E}} \Gamma$ the isotropy group $\Gamma_{x}=\{g \in \Gamma \mid g \cdot x=$ $x\}$ is finite and for every finite subgroup $H \subset \Gamma$ the fixed-point set $\underline{\mathbf{E}} \Gamma^{H}$ is contractible. It has the universal property that for every (numerably) proper $\Gamma$-space $X$ there is a unique (up to $\Gamma$-homotopy) equivariant map $X \rightarrow \underline{\mathbf{E}} \Gamma$.

Using the equivariant setup, the Rosenberg index $\alpha$ is the map from the top left to the bottom right in the following commutative diagram:

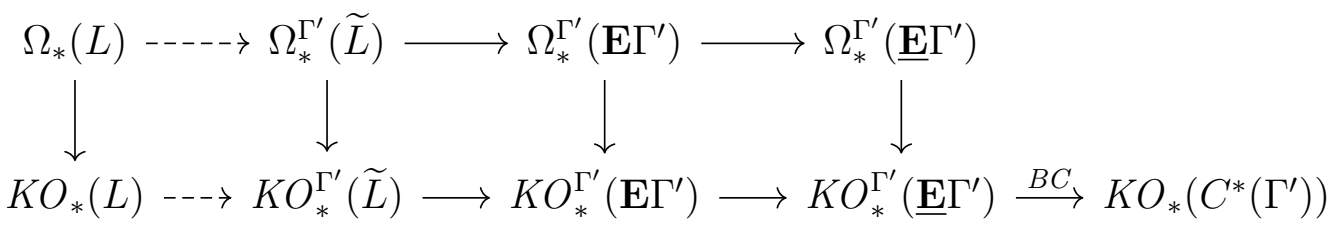

Here the dashed arrows denote the transfer to the equivariant theories, the vertical arrows denote the maps from the natural transformation, $B C$ is the Baum-Connes map and all other horizontal arrows are induced by the classifying topological maps.

We want to show that the fundamental class $[L, s, i d] \in \Omega_{*}(L)$ (s being a Spin-structure on $L)$ is mapped to zero in $K O_{*}\left(C^{*}\left(\Gamma^{\prime}\right)\right)$. By the preceding commutative diagram it suffices to show that its image in $\Omega_{*}^{\Gamma^{\prime}}\left(\underline{\mathbf{E}} \Gamma^{\prime}\right)$ is zero. 
Consider the following commutative diagram where all morphisms are induced by the topological maps and the $\Gamma^{\prime}$-action on the spaces in the bottom row factors through $\Gamma$ :

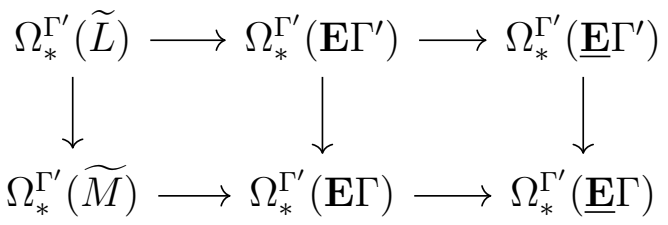

$\underline{\mathbf{E}} \Gamma$ with the action $\Gamma^{\prime} \rightarrow \Gamma \curvearrowright \underline{\mathbf{E}} \Gamma$ is a proper $\Gamma^{\prime}$-CW-complex because the kernel of the extension $\Gamma^{\prime} \rightarrow \Gamma$ is finite. Because it satisfies the necessary requirements on the isotropy groups and the fixed point sets, it is therefore also a model for $\underline{\mathbf{E}} \Gamma^{\prime}$. By the universal property of $\underline{\mathbf{E}} \Gamma^{\prime}$ the map $\underline{\mathbf{E}} \Gamma^{\prime} \rightarrow \underline{\mathbf{E}} \Gamma$ can then be taken to be the identity and therefore induces an isomorphism on the bordism groups.

By the commutativity of the diagram it therefore suffices to show that the image of $[\widetilde{L}]_{\text {Spin }}$ vanishes in $\Omega^{\Gamma^{\prime}}(\widetilde{M})$, i.e., that the singular manifold $\widetilde{L} \rightarrow \widetilde{M}$ is $\Gamma^{\prime}$-equivariantly Spin-null-bordant.

The key observation is now that the universal covering $\widetilde{L}$ can be viewed as an $S^{1}$-bundle over the universal covering $\widetilde{M}$. Let $L^{\prime}$ be the pullback of $p_{\widetilde{M}}: \widetilde{M} \rightarrow M$ and $\pi_{L}: L \rightarrow M:$

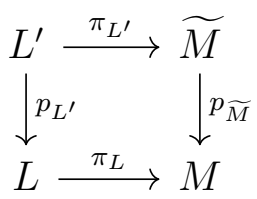

Then $L^{\prime} \rightarrow L$ is the covering corresponding to the subgroup $\mathbb{Z}_{n} \subset \Gamma^{\prime}$. And at the same time $L^{\prime} \rightarrow \widetilde{M}$ is the $S^{1}$-bundle corresponding to $x_{L^{\prime}}=p_{\widetilde{M}}{ }^{*}\left(x_{L}\right)$ (where $x_{L} \in H^{2}(M ; \mathbb{Z}$ ) classifies the bundle $L$ ).

Lemma 4.3.1 implies $x_{L^{\prime}} \in n \cdot H^{2}(\widetilde{M} ; \mathbb{Z})$. Also, $H_{1}(\widetilde{M} ; \mathbb{Z})=0$ is torsionfree, and by the universal coefficient theorem the same is true for $H^{2}(\widetilde{M} ; \mathbb{Z})$. Therefore, let $L^{\prime \prime}$ be the unique $S^{1}$-bundle over $\widetilde{M}$ classified by $x_{L^{\prime \prime}}=1 / n \cdot x_{L^{\prime}} \in$ $H^{2}(\widetilde{M} ; \mathbb{Z})$. Then there is a well defined map $L^{\prime \prime} \rightarrow L^{\prime \prime} / \mathbb{Z}_{n} \cong L^{\prime}$ given by quotienting out the action of $\mathbb{Z}_{n} \subset S^{1} \curvearrowright L^{\prime \prime}$. This map is a covering. Because $\widetilde{M}$ is simply connected, the fundamental group $\pi_{1}\left(L^{\prime \prime}\right)$ is generated by the inclusion of the fiber $S^{1} \rightarrow L^{\prime \prime}$. But this inclusion maps to a null-homotopic circle under the covering map $L^{\prime \prime} \rightarrow L^{\prime}$, thus it is itself null-homotopic. This means that $L^{\prime \prime}$ is simply connected and hence the universal covering of $L^{\prime}$ and of $L$.

Now, the $S^{1}$-bundle $\widetilde{L} \rightarrow \widetilde{M}$ is the boundary of the corresponding disk bundle $\bar{L}$. The action $\Gamma^{\prime} \curvearrowright \widetilde{L}$ extends to $\bar{L}$, on the zero-section of the disk bundle it is just $\Gamma^{\prime} \rightarrow \Gamma \curvearrowright \widetilde{M}$. Because both $\widetilde{L}$ and $\bar{L}$ are connected and the $\Gamma^{\prime}$-action on $\widetilde{L}$ respects the orientation, it also respects the orientation on 
$\bar{L}$. It remains to check that $\bar{L}$ can be equipped with a $\Gamma^{\prime}$-Spin-structure that restricts on the boundary to the Spin-structure on $\widetilde{L}$.

First we check that $\bar{L}$ admits a non-equivariant Spin-structure. Indeed, this is true for the disk bundle $\hat{L}$ of the lifted $S^{1}$-bundle $L^{\prime}$. Then, because $n$ is odd (this is where the assumption that $\widetilde{M}$ is non-Spin comes in through Lemma 4.3.1), $x_{L^{\prime \prime}}=x_{L^{\prime}} \bmod 2$. It follows (compare Lemma 4.2.2)

$$
w_{2}(T \bar{L})=\left(\pi_{T \bar{L}}\right)^{*}\left(w_{2}(T \widetilde{M})+w_{2}\left(\bar{L}^{\circ}\right)\right)=\left(\pi_{T \bar{L}}\right)^{*}\left(w_{2}(T \widetilde{M})+w_{2}\left(\hat{L}^{\circ}\right)\right)=0,
$$

hence $\bar{L}$ is Spin. Because both $\bar{L}$ and $\widetilde{L}$ are simply connected, there is, when neglecting the $\Gamma^{\prime}$ action, exactly one Spin-structure $\bar{s}: \bar{P}_{S p i n} \rightarrow \bar{P}_{S O}$ on $\bar{L}$, and its boundary is the unique Spin-structure $\widetilde{s}: \widetilde{P}_{\text {Spin }} \rightarrow \widetilde{P}_{S O}$ on $\widetilde{L}$. After stabilizing we may assume that $\bar{P}_{\text {Spin }}$ and $\widetilde{P}_{\text {Spin }}$ are $\operatorname{Spin}(k)$ principal bundles for the same $k$. Then $\widetilde{P}_{\text {Spin }} \subset \bar{P}_{\text {Spin }}$ and taking the boundary of $\bar{s}$ just means restricting the map to $\widetilde{P}_{S p i n}$. We have to lift the $\Gamma^{\prime}$-action from $\bar{P}_{S O}$ to $\bar{P}_{S p i n}$ such that it coincides with the given (stabilized) $\Gamma^{\prime}$-action on $\widetilde{P}_{\text {Spin }}$.

But both $\bar{s}$ and $\widetilde{s}$ are the universal coverings of $\bar{P}_{S O}$ and $\widetilde{P}_{S O}$, respectively. Therefore, if we fix any point $v_{0} \in \widetilde{P}_{\text {Spin }} \subset \bar{P}_{\text {Spin }}$, then for every $\gamma \in \Gamma^{\prime}$ the assignment $v_{0} \mapsto \gamma \cdot v_{0}$ extends uniquely to a map on both $\bar{P}_{\text {Spin }}$ and $\widetilde{P}_{\text {Spin }}$. The resulting map $\Gamma^{\prime} \times \bar{P}_{\text {Spin }} \rightarrow \bar{P}_{\text {Spin }}$ is a group action because it is one on the orbit of $v_{0}$. It agrees with the given action on $\widetilde{P}_{\text {Spin }}$ and it makes the map $\bar{P}_{\text {Spin }} \rightarrow \bar{P}_{S O}$ equivariant.

This finishes the proof of the first part of Theorem 1.3:

Theorem 4.7.5. Let $M$ be a closed Spin ${ }^{c}$ manifold such that $\widetilde{M}$ is not Spin and let $\pi_{L}: L \rightarrow M$ be an $S^{1}$-bundle that is Spin.

Then the Rosenberg index of $L$ inside $K O\left(C^{*}\left(\pi_{1}(L)\right)\right)$ always vanishes.

Remark 4.7.6. By interpreting the cohomology class $x_{L^{\prime}}$ as a $\Gamma$-invariant element of $\operatorname{Hom}\left(\pi_{2}(M), n \mathbb{Z}\right)$ one sees that $x_{\widetilde{L}}=1 / n \cdot x_{L^{\prime}} \in \operatorname{Hom}\left(\pi_{2}(M), \mathbb{Z}\right)$ is also $\Gamma$-invariant. This, however, does not necessarily mean that $x_{\widetilde{L}} \in H^{2}(\widetilde{M} ; \mathbb{Z})$ is the pullback of an element $y \in H^{2}(M ; \mathbb{Z}$ ) (which would be equivalent to the corresponding $S^{1}$-bundle being a pullback from a bundle over $M$ ).

In the general setting $p_{\tilde{X}}: \widetilde{X} \rightarrow X$ there are two possible reasons why a $\pi_{1}(X)$-invariant element $x \in H^{2}(\tilde{X} ; \mathbb{Z})$ might not be a pullback of an element in $H^{2}(X ; \mathbb{Z})$. The first reason is that $x$ might have non-zero values on $\operatorname{ker}\left(p_{\widetilde{X}_{*}}: H_{2}(\widetilde{X} ; \mathbb{Z}) \rightarrow H_{2}(X ; \mathbb{Z})\right)$. This can happen when the classifying map $u_{X}: X \rightarrow \mathbf{B} \pi_{1}(X)$ does not induce a surjection on $H_{3}$, for example when $X$ is the 2-skeleton of $\mathbb{T}^{3}$. For more information see [Bro82], Theorem VII.7.9 and Exercise 6 on the Cartan-Leray spectral sequence. This problem, however, does not arise in the above setting: Because the coefficient ring $\mathbb{Z}$ is torsionfree, $x_{\widetilde{L}}$ evaluates to zero exactly when $n \cdot x_{\widetilde{L}}=x_{L^{\prime}}$ evaluates to zero and $x_{L^{\prime}}$ is the pullback of $x_{L}$. 
If $x$ evaluates to zero on $\operatorname{ker}\left(p_{\tilde{X}_{*}}: H_{2}(\widetilde{X} ; \mathbb{Z}) \rightarrow H_{2}(X ; \mathbb{Z})\right)$, it defines an element of $\operatorname{Hom}\left(\operatorname{Im}\left(p_{\widetilde{X} *}: H_{2}(\widetilde{X} ; \mathbb{Z}) \rightarrow H_{2}(X ; \mathbb{Z})\right), \mathbb{Z}\right)$. The second problem that may occur is that this homomorphism might not extend to $H_{2}(X ; \mathbb{Z})$.

By the Hurewicz isomorphism $\operatorname{Im}\left(p_{\widetilde{X} *}: H_{2}(\widetilde{X} ; \mathbb{Z}) \rightarrow H_{2}(X ; \mathbb{Z})\right)$ is the subgroup of $H_{2}(X ; \mathbb{Z})$ that can be represented by singular 2-spheres, and this subgroup in turn is precisely $\left.\operatorname{ker}\left(u_{X *}: H_{2}(X ; \mathbb{Z}) \rightarrow H_{2}\left(\mathbf{B} \pi_{1}(X)\right) ; \mathbb{Z}\right)\right)$. Therefore, the second problem may occur if $\left.H_{2}\left(\mathbf{B} \pi_{1}(X)\right) ; \mathbb{Z}\right)$ contains torsion.

As an example take as $X$ the 2 -skeleton of the classifying space for

$$
G=\mathbb{Z} \times \mathbb{Z}_{n}=\left\{z, a \mid z a z^{-1} a^{-1}, a^{n}\right\}
$$

and let $y \in H^{2}(X ; \mathbb{Z})$ evaluate to 1 on the relation $z a z^{-1} a^{-1}$. Then $1 / n \cdot p_{\widetilde{X}}{ }^{*}(y) \in$ $H^{2}(\widetilde{X} ; \mathbb{Z})$ is not the pullback of any $y^{\prime} \in H^{2}(X ; \mathbb{Z})$.

\subsection{Example for a non-psc circle bundle}

Finally, we give an example where the $S^{1}$-bundle produced by our construction does not admit a psc metric. This means that the bundle construction does not in itself remove all information pertaining to positive scalar curvature. Instead, it will, in some cases, lead to non-psc Spin manifolds with vanishing index-theoretical obstruction. Such manifolds have been known to exist for some time. They are, however, considered to be the exception. The bundle construction might be used to construct a class of examples where this behavior occurs.

The first example for a non-psc manifold with vanishing Rosenberg index was given by Schick in Sch98. Schick realizes an element of odd order inside $\Omega_{5}^{\text {Spin }}\left(\mathbf{B}\left(\mathbb{Z}^{4} \times \mathbb{Z}_{3}\right)\right)$ by a singular manifold $(M, f)$. After doing surgery one can assume that $f$ is a classifying map for $\pi_{1}(M)$. Then, because $K O_{*}\left(C^{*}\left(\mathbb{Z} \times \mathbb{Z}_{3}\right)\right)$ does not contain odd torsion, $\alpha\left([M]_{\text {Spin }}\right)=0 \in K O_{5}\left(C^{*}\left(\mathbb{Z} \times \mathbb{Z}_{3}\right)\right)$. But with the minimal hypersurface method it can be shown that $M$ is non-psc.

Our circle bundle situation is very similar to this counterexample and we can use essentially the same proof.

Example 4.8.1. Let $M=\mathbb{T}^{4} \# \mathbb{C} P^{2}$ and $x \in H^{2}(M ; \mathbb{Z})$ the element that comes from the generator of $H^{2}\left(\mathbb{C} P^{2} ; \mathbb{Z}\right)=\mathbb{Z}$. The circle bundle $L \rightarrow M$ given by $3 x$ is a Spin manifold with fundamental group $G=\mathbb{Z}^{4} \times \mathbb{Z}_{3}$.

To show that $L$ is non-psc, we first calculate $\left(u_{L}\right)_{*}([L]) \in H_{5}(\mathbf{B} G ; \mathbb{Z})$. Let $S=S^{3}$ be the transversal sphere of the connected sum $M=\mathbb{T}^{4} \# \mathbb{C} P^{2}$ and let $S \times[0,1]$ be a neighborhood of $S$. Because $L$ is a trivial $S^{1}$-bundle over $S \times[0,1]$, there is a bordism $W$ between $L$ and an $S^{1}$-bundle $L^{\prime}=L_{1} \sqcup L_{2} \rightarrow$ $\mathbb{T}^{4} \sqcup \mathbb{C} P^{2}$. Namely, $W$ is obtained from $M \times[0,1]$ by attaching $D^{4} \times[0,1] \times S^{1}$ along $S \times[0,1] \times S^{1} \times\{0\} \subset M \times[0,1]$. The bundle $L^{\prime}$ again corresponds to 
$3 x \in H^{2}\left(\mathbb{T}^{4} \sqcup \mathbb{C} P^{2} ; \mathbb{Z}\right)$, meaning, in particular, that $L_{1}$ is the trivial bundle over $\mathbb{T}^{4}$. The inclusion $M \subset W$ is 2-connected and thus the map $u_{L}: L \rightarrow \mathbf{B} G$ extends to $u_{W}: W \rightarrow \mathbf{B} G$.

In $H_{5}(W ; \mathbb{Z})$ one has $0=\partial[W]=i_{0 *}([L])-i_{1 *}\left(\left[L_{1}\right]\right)-i_{2 *}\left(\left[L_{2}\right]\right)$ (where $i_{0}, i_{1}, i_{2}$ are the obvious inclusions). Hence

$$
\left(u_{L}\right)_{*}([L])=\left(u_{W} \circ i_{0}\right)_{*}([L])=\left(u_{W} \circ i_{1}\right)_{*}\left(\left[L_{1}\right]\right)+\left(u_{W} \circ i_{2}\right)_{*}\left(\left[L_{2}\right]\right) .
$$

Now $\left(u_{W} \circ i_{1}\right)_{*}\left(\left[L_{1}\right]\right)=\left[\mathbb{T}^{4}\right] \times y \in H^{5}(\mathbf{B} G ; \mathbb{Z})$ where $y \in H_{1}\left(\mathbf{B} \mathbb{Z}_{3} ; \mathbb{Z}\right)$ is a generator. And because $\mathbb{C} P^{2}$ is simply connected, $u_{W} \circ i_{2}$ factors through $\mathbf{B} \mathbb{Z}_{3} \times p t \subset \mathbf{B} G$, such that $\left(u_{W} \circ i_{2}\right)_{*}\left(\left[L_{2}\right]\right) \in H_{5}\left(\mathbf{B} \mathbb{Z}_{3}\right) \subset H_{5}(\mathbf{B} G)$. It follows that if $a_{1}, a_{2}, a_{3} \in H^{1}(\mathbf{B} G ; \mathbb{Z})$ correspond to the first three $S^{1}$ factors of $\mathbf{B} G$, then

$$
a_{1} \cap\left(a_{2} \cap\left(a_{3} \cap\left(u_{L}\right)_{*}([L])\right)\right) \neq 0 \in H_{2}(\mathbf{B} G ; \mathbb{Z}) .
$$

On the other hand, there is the following corollary of the minimal hypersurface method ([Sch98, 1.6]):

Theorem 4.8.2. Let $X$ be any topological space and let

$$
H_{m}^{+}(X)=\left\{f_{*}([M]) \in H_{m}(X ; \mathbb{Z}) \mid f: M \rightarrow X \text { and } M \text { is a psc manifold }\right\} .
$$

For $3 \leq m \leq 7$ taking cap product with any $a \in H^{1}(X ; \mathbb{Z})$ maps $H_{m}^{+}(X)$ into $H_{m-1}^{+}(X)$.

By the above theorem, if $L$ were psc, then $0 \neq a_{1} \cap\left(a_{2} \cap\left(a_{3} \cap f_{*}([L])\right)\right) \in$ $H_{2}^{+}(\mathbf{B} G)$. But $H_{2}^{+}(\mathbf{B} G)$ is trivial because $S^{2}$ is the only psc 2-manifold and all maps $S^{2} \rightarrow \mathbf{B} G$ are null-homotopic. Therefore, $L$ does not allow a metric of positive scalar curvature. 


\section{The codimension-2 transfer}

\subsection{Motivation}

When the Spin manifold $M$ has a suitable codimension-2 submanifold $N$, an obstruction against positive scalar curvature on $M$ can also be obtained by applying the Rosenberg index $\alpha$ to $N$ instead of $M$. Building on the work of Gromov and Lawson (GL83) Hanke, Pape and Schick showed the following ([HPS15]):

Theorem 5.1.1. Let $M$ be an n-dimensional Spin manifold and $N \subset M$ a connected submanifold of codimension 2 with trivial normal bundle $\nu(N)$. Assume that $\pi_{1}(N) \rightarrow \pi_{1}(M)$ is injective and $\pi_{2}(N) \rightarrow \pi_{2}(M)$ surjective.

Then $\alpha\left([N]_{\text {Spin }}\right) \in K O_{n-2}\left(C^{*}\left(\pi_{1}(N)\right)\right)$ is an obstruction against the existence of psc-metrics on $M$.

Remark 5.1.2. Actually, the statement given in [HPS15] assumes that $\pi_{2}(M)$ is trivial. But the proof still works for $\pi_{2}(N) \rightarrow \pi_{2}(M)$ surjective, and even for $\pi_{2}(N) \oplus \pi_{2}(M \backslash N) \rightarrow \pi_{2}(M)$ surjective.

Indeed, the only place where the requirement $\pi_{2}(M)=0$ is needed is to show that the map $\pi_{1}(\partial D \nu(N)) \rightarrow \pi_{1}(\bar{M} \backslash D \nu(N))$ is an injection, where $D \nu(N)$ denotes the disk bundle inside the normal bundle of $N \subset M, \partial D \nu$ denotes its boundary, $\bar{M} \rightarrow M$ is the covering corresponding to the subgroup $\pi_{1}(N) \subset \pi_{1}(M)$ and the inclusion $D \nu(N) \rightarrow \bar{M}$ is a lift of the inclusion $D \nu(N) \subset M$.

If $(a, t) \in \pi_{1}(\partial D \nu)=\pi_{1}(N) \times \mathbb{Z}$ lies in the kernel of this map, then commutativity of the van Kampen pushout diagram

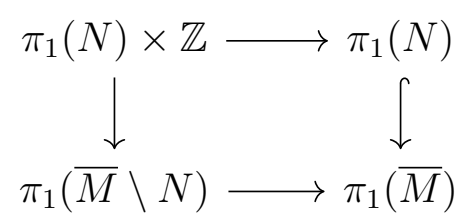

implies $a=0$. But if $t \neq 0$, then the null-homotopy of $(0, t)$ inside $\bar{M} \backslash D \nu$ together with the null-homotopy inside $D^{2} \subset D \nu$ forms a singular sphere $\phi: S^{2} \rightarrow \bar{M}$. The homology class defined by this sphere has non-trivial intersection with the homology class given by $N$. The contradiction now follows from the weakened condition that $\pi_{2}(N) \oplus \pi_{2}(M \backslash N) \rightarrow \pi_{2}(M)$ is surjective. (Because $\pi_{2}(N) \rightarrow \pi_{2}(M)$ factors through $\pi_{2}(M \backslash N)$, this is actually equivalent to $\pi_{2}(M \backslash N) \rightarrow \pi_{2}(M)$ being surjective.)

The proof also uses $\pi_{2}(M)=0$ to show that $H_{2}(\bar{M}, N ; \mathbb{Z})=0$. But what is needed is only $H_{2}(\bar{M} ; \mathbb{Z}) \rightarrow H_{2}(\bar{M}, N ; \mathbb{Z})$ surjective, and this follows from $H_{1}(N ; \mathbb{Z}) \rightarrow H_{1}(\bar{M} ; \mathbb{Z})$ injective and the long exact sequence of homology. 
It is unclear how the obstruction $\alpha\left([N]_{\text {Spin }}\right)$ relates to the obstruction $\alpha\left([M]_{\text {Spin }}\right)$. For example, when $\alpha[M]_{\text {Spin }}=0$ and the Baum-Connes map is injective for $\pi_{1}(M)$, then by Theorem 2.4.6 $M \times B t \times \cdots \times B t$ is psc. Then by Theorem 5.1.1 it holds $\alpha\left([N]_{\text {Spin }}\right)=\alpha([N \times B t \times \cdots \times B t])=0$. But it is not known if in general $\alpha\left([M]_{\text {Spin }}\right)=0$ implies $\alpha\left([N]_{\text {Spin }}\right)=0$.

The best possible result would be if there existed transfer homomorphisms $t r_{\text {top }}: K O_{*}(M) \rightarrow K O_{*-2}(N), \operatorname{tr}_{C^{*}}: K O_{*}\left(C^{*}\left(\pi_{1}(M)\right)\right) \rightarrow K O_{*-2}\left(C^{*}\left(\pi_{1}(N)\right)\right)$ such that $\operatorname{tr}_{\text {top }}\left([M]_{K O}\right)=[N]_{K O}$ and the diagram

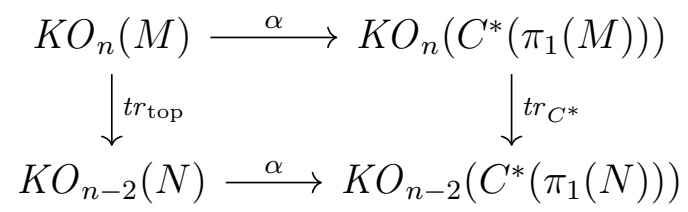

commutes. The obvious candidate for the map $t r=t r_{\text {top }}$ is the composition

$$
K O_{*}(M) \rightarrow K O_{*}(M, M \backslash D \nu(N)) \cong K O_{*}(D \nu(N), \partial D \nu(N)) \rightarrow K O_{*-2}(N)
$$

where the first map is the forgetful map and the second one is the Thom isomorphism, obtained by taking the cap product with $f^{*}(e)$, where $e \in K O^{2}\left(D^{2}, S^{1}\right)$ is the doubly suspended unit and $f:(D \nu(N), \partial D \nu(N)) \rightarrow\left(D^{2}, S^{1}\right)$ is the trivialization.

A suitable map $\operatorname{tr}_{C^{*}}$ has been found by Zeidler ([Zei17, 1.8]) in the case where $N \subset M$ is a codimension-1 submanifold. In the codimension- 2 case no appropriate map has been found so far.

Since the map $\alpha$ factors through $K O\left(\mathbf{B} \pi_{1}(\cdot)\right)$ and $K O^{\pi_{1}(\cdot)}\left(\underline{\mathbf{E}} \pi_{1}(\cdot)\right)$, we consider the intermediate step of extending the transfer map $\operatorname{tr}_{\text {top }}$ on the topological side to $K O_{*}\left(\mathbf{B} \pi_{1}(M)\right) \rightarrow K O_{*-2}\left(\mathbf{B} \pi_{1}(N)\right)$ and to $K O_{*}^{\pi_{1}(M)}\left(\underline{\mathbf{E}} \pi_{1}(M)\right) \rightarrow$ $K O_{*-2}^{\pi_{1}(N)}\left(\underline{\mathbf{E}} \pi_{1}(N)\right)$. If $\pi_{1}(M)$ satisfies the Baum-Connes Conjecture, this will also give the homomorphism $t r_{C^{*}}$. The first extension can be done by generalizing a construction of Engel ([Eng17]). We do this in Section 5.3. The second extension is made in Section 5.4. Both extensions will be part of the upcoming article [NSZ] of Nitsche, Schick and Zeidler.

\subsection{Examples and restrictions on the funda- mental groups}

The situations where Theorem 5.1.1 can be applied include the following two classes of examples:

Example 5.2.1. The lowest-dimensional examples (except for $N=\{p t\}$ ) consist of the embedding of $N=S^{1}$ into an orientable 3-dimensional manifold $M$. From the $\mathrm{Wu}$ formulas it follows that $M$ is automatically Spin. One still has to require that the inclusion $N \rightarrow M$ induces an injection on $\pi_{1}$, and that $\pi_{2}(M)=0$ (which implies that $M$ is aspherical, see Lemma 5.3.9). 
These examples were investigated by Gromov and Lawson in GL83, section 8.

Example 5.2.2. A second class of examples consists of $M$ a fiber bundle with base space $\Sigma$ an orientable surface of genus $>0$, and $N \rightarrow M$ the inclusion of a fiber. Because the base space of the bundle is aspherical, the long exact sequence of homotopy groups

$$
\longrightarrow \pi_{2}(N) \longrightarrow \pi_{2}(M) \longrightarrow \pi_{2}(\Sigma) \longrightarrow \pi_{1}(N) \longrightarrow \pi_{1}(M) \longrightarrow
$$

shows that the inclusion $N \rightarrow M$ always induces a surjection on $\pi_{2}$ and an injection on $\pi_{1}$. We have to require that $M$ is Spin, then $N$ is also Spin.

Next, we examine the restrictions that the conditions $D \nu(N) \cong N \times D^{2}$, $\pi_{1}(N) \rightarrow \pi_{1}(M)$ injective and $\pi_{2}(N) \rightarrow \pi_{2}(M)$ surjective pose on the fundamental groups of $N, M$ and $M \backslash N$.

Lemma 5.2.3. The conditions $\pi_{1}(N) \rightarrow \pi_{1}(M)$ injective, $\pi_{2}(N) \rightarrow \pi_{2}(M)$ surjective imply that the groups $\pi_{1}(N), \pi_{1}(\partial D \nu(N)) \cong \pi_{1}(N) \times \mathbb{Z}, \pi_{1}(M)$ and $\pi_{1}(M \backslash N)$ fit into the following pushout diagram:

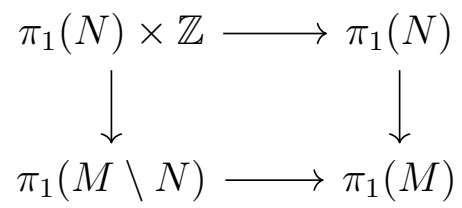

The vertical maps in the diagram are injections.

Proof. The existence of the pushout diagram follows from van Kampen's Theorem applied to the decomposition $M=D \nu(N) \cup(M \backslash D \nu(N))$. The argument that the map $\pi_{1}\left(N \times S^{1}\right) \rightarrow \pi_{1}(M \backslash N)$ is injective is the same as the one given in Remark 5.1.2.

This leads to the question which pushout diagrams can occur.

Lemma 5.2.4. For any injective group homomorphism $i: H \rightarrow G$ of finitely presented groups there is a manifold pair $N \subset M$ of closed connected Spin manifolds to which Theorem 5.1.1 can be applied, with $\pi_{1}(N)=H, \pi_{1}(M)=G$ and the map $\pi_{1}(N) \rightarrow \pi_{1}(M)$ given by $i$.

Proof. By using 0-, 1- and 2-surgery one can construct a high-dimensional Spin-manifold $N^{\prime}$ with $\pi_{1}\left(N^{\prime}\right)=H$ and $\pi_{2}\left(N^{\prime}\right)=0$. The surgery can be performed in such a way that the tangent bundle of $N^{\prime}$ is stably trivializable. In particular, $N^{\prime}$ is Spin. Now let $N=N^{\prime} \times S^{3}, M^{\prime}=N^{\prime} \times S^{5}$ and $N \rightarrow M^{\prime}$ induced by an embedding $S^{3} \rightarrow S^{5}$. Since $S^{5} \backslash S^{3} \simeq S^{1}$ and the induced map $\pi_{1}\left(\partial D \nu\left(S^{3} \subset S^{5}\right)\right) \rightarrow \pi_{1}\left(S^{5} \backslash S^{3}\right)$ is an isomorphism, $\pi_{1}(\partial D \nu(N)) \rightarrow$ $\pi_{1}\left(M^{\prime} \backslash D \nu(N)\right)$ is also an isomorphism. 
Next, we apply 0- and 1- surgery to $M^{\prime}$ - away from $D \nu(N)$ - such that $\pi_{1}\left(M^{\prime} \backslash D \nu(N)\right)=(G * \mathbb{Z}) /\langle[H, \mathbb{Z}]\rangle$ and $\pi_{1}(\partial D \nu(N)) \rightarrow \pi_{1}\left(M^{\prime} \backslash D \nu(N)\right)$ is the canonical map. Finally, we apply 2-surgery to $M^{\prime}$ away from $D \nu(N)$ to ensure $\pi_{2}\left(M^{\prime} \backslash D \nu(N)\right)=0$ and let $M$ be the resulting manifold. Then $\pi_{1}(M)=G$ and $\pi_{1}(N) \rightarrow \pi_{1}(M)$ is the prescribed map $i$. Again, the surgeries can be made in such a way that $M^{\prime}$ is Spin. It remains to check that $\pi_{2}(M)=0$.

Consider the Mayer-Vietoris sequence for the decomposition of $\widetilde{M}$ as the union of $A=p_{\widetilde{M}}^{-1}(D \nu(N))$ and $B$ the closure of $\widetilde{M} \backslash A$.

$$
\rightarrow H_{2}(A ; \mathbb{Z}) \oplus H_{2}(B ; \mathbb{Z}) \rightarrow H_{2}(\widetilde{M} ; \mathbb{Z}) \stackrel{\partial_{2}}{\rightarrow} H_{1}(A \cap B ; \mathbb{Z}) \rightarrow H_{1}(B ; \mathbb{Z}) \rightarrow
$$

$A \cap B$ is homotopy equivalent to a disjoint union of circles, with one circle $S_{[g]}$ for each coset $[g] \in \pi_{1}(M) / \pi_{1}(N)$. Since $B$ is the pullback of $\widetilde{M}$ along the inclusion $M \backslash D \nu(N) \rightarrow M$

$$
\pi_{1}(B)=\operatorname{ker}\left(\pi_{1}(M \backslash D \nu(N)) \rightarrow \pi_{1}(M)\right),
$$

which in this example is the free group with one generator $a_{[g]}=g z g^{-1}$ for every coset $[g] \in \pi_{1}(M) / \pi_{1}(N)$ (here $z=1 \in \mathbb{Z}$ ). The image of the circle $S_{[g]}$ under the inclusion $A \cap B \rightarrow B$ represents the generator $a_{[g]}$.

This means that the map $H_{1}(A \cap B ; \mathbb{Z}) \rightarrow H_{1}(B ; \mathbb{Z})$ is injective, and hence the boundary map $\partial_{2}$ is zero. By exactness of the Mayer-Vietoris sequence and the Hurewicz Theorem applied to $\pi_{2}(\widetilde{M})$, this means that $\pi_{2}(M) \cong H_{2}(\widetilde{M} ; \mathbb{Z})$ is a quotient of $H_{2}(A ; \mathbb{Z}) \oplus H_{2}(B ; \mathbb{Z})=H_{2}(B ; \mathbb{Z})$. Since the construction of $M$ gives $\pi_{2}(B)=\pi_{2}(M \backslash D \nu(N))=0$, the classifying space for $\pi_{1}(B)$ can be obtained from $B$ by attaching cells of dimension $>3$. Hence $H_{2}(B ; \mathbb{Z})=$ $H_{2}\left(\mathbf{B} \pi_{1}(B) ; \mathbb{Z}\right)=0$. This means that the quotient $\pi_{2}(M)$ is also trivial, finishing the proof.

The examples produced in the preceding proof are psc manifolds and therefore have trivial Rosenberg indexes. However, Lemma 5.2.4 shows that if we want to construct the transfer map $K O_{*}\left(C^{*}\left(\pi_{1}(M)\right) \rightarrow K O_{*-2}\left(C^{*}\left(\pi_{1}(N)\right)\right)\right.$ on the level of $C^{*}$-algebras, we cannot rely on any special properties of the groups involved.

When including the group $\pi_{1}(M \backslash N)$, the question of which pushout diagrams can be realized becomes more difficult. For example, the following pushout diagram cannot be realized:

Example 5.2.5. Consider the pushout diagram of groups

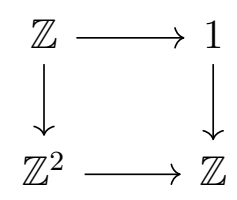

where the left vertical map is the inclusion into one of the factors. Assume that the diagram is realized by a suitable inclusion $N \rightarrow M$. Let $Y$ be the 
CW-complex obtained from $M \backslash D \nu(N)$ by killing the second homotopy group with 3-cells, and let $X=D \nu(N) \cup_{\partial D \nu(N)} Y$. Then $\pi_{2}(X)=0$ because we require that $\pi_{2}(N) \rightarrow \pi_{2}(M)$ is surjective and $\pi_{2}(N) \cong \pi_{2}(\partial D \nu(N))$.

Since $\mathbf{B} \pi_{1}(M)$ and $\mathbf{B} \pi_{1}(M \backslash N)$ can be obtained from $X$ and $Y$, respectively, by attaching only cells of dimension $\geq 4$,

$$
\begin{aligned}
H_{2}(X ; \mathbb{Z}) & =H_{2}\left(\mathbf{B} \pi_{1}(X) ; \mathbb{Z}\right)=H_{2}\left(\mathbf{B} \pi_{1}(M) ; \mathbb{Z}\right)=H_{2}(\mathbf{B} \mathbb{Z} ; \mathbb{Z})=0, \\
H_{2}(Y ; \mathbb{Z}) & =H_{2}\left(\mathbf{B} \pi_{1}(Y) ; \mathbb{Z}\right)=H_{2}\left(\mathbf{B} \pi_{1}(M \backslash N) ; \mathbb{Z}\right)=H_{2}\left(\mathbf{B} \mathbb{Z}^{2} ; \mathbb{Z}\right)=\mathbb{Z} .
\end{aligned}
$$

But since $N$ is simply connected, the map $H_{2}\left(N \times S^{1} ; \mathbb{Z}\right) \rightarrow H_{2}\left(N \times D^{2} ; \mathbb{Z}\right)$ is an isomorphism. This is a contradiction to the exactness of the Mayer-Vietoris sequence of the decomposition of $X=D \nu(N) \cup Y$

$$
\longrightarrow H_{2}\left(N \times S^{1} ; \mathbb{Z}\right) \longrightarrow H_{2}\left(N \times D^{2} ; \mathbb{Z}\right) \oplus \underbrace{H_{2}(Y ; \mathbb{Z})}_{\mathbb{Z}} \longrightarrow \underbrace{H_{2}(X ; \mathbb{Z})}_{0} \longrightarrow
$$

Smith ([Smi78]) investigated which groups can occur for $\pi_{1}(M \backslash N)$ in the case that $N \rightarrow M$ induces an isomorphism of fundamental groups. The following is his main result specialized to the case of a trivial normal bundle.

Theorem 5.2.6. Let $f: N \rightarrow M$ be a codimension-2 inclusion of compact connected manifolds with trivial normal bundle, such that $\pi_{1}(N) \rightarrow \pi_{1}(M)$ is an isomorphism, $\pi_{2}(N) \rightarrow \pi_{2}(M)$ is a surjection and $\operatorname{dim}(N) \geq 3$.

Then a finitely presented group $G$ can be realized as the fundamental group of the complement of an embedding homotopic to $f$ iff $G$ fits into an extension $q: G \rightarrow \pi_{1}(N) \times \mathbb{Z}$ with split $s$ such that $q^{-1}(1 \times \mathbb{Z})$ is normally generated by $s(0,1)$ and $H_{2}\left(q^{-1}(1 \times \mathbb{Z}) ; \mathbb{Z}\right)=0$.

Using surgery as in the proof of Lemma 5.2.4 the required initial inclusion $f: N \rightarrow M$ can be constructed for every fundamental group $H=\pi_{1}(N)=$ $\pi_{1}(M)$. Therefore, it follows:

Corollary 5.2.7. Let $H$ and $G$ be finitely presented groups. Then the following are equivalent:

1. There exists a codimension-2 inclusion of compact connected Spin manifolds $N \subset M$ with trivial normal bundle, such that $\pi_{1}(N)=\pi_{1}(M)=H$, $\pi_{1}(M \backslash N)=G$ and $\pi_{2}(N) \rightarrow \pi_{2}(M)$ surjective.

2. $G$ is an extension $q: G \rightarrow H \times \mathbb{Z}$ with split $s$ such that $q^{-1}(1 \times \mathbb{Z})$ is normally generated by $s(0,1)$ and $H_{2}\left(q^{-1}(1 \times \mathbb{Z}) ; \mathbb{Z}\right)=0$.

\subsection{Extending the transfer map}

Adapting the method of Engel ([Eng17]), the codimension-2 transfer map can be extended to $\mathbf{B}\left(\pi_{1}(M)\right)$. The idea for this goes back to Schick. In this 
section we give a proof that works for general equivariant homology theories on $\Gamma$-CW-complexes. In addition to the usual axioms (see [Lüc02a]) we will, however, demand that the homology theory allows an operation that we call "lf-restriction" (the "lf" stands for locally finite).

Definition 5.3.1. Let $\Gamma$ be a countable discrete group, let $\pi$ be a subgroup, and let $Y$ be a $\Gamma$-CW-complex. A $\pi$-invariant $\mathrm{CW}$-subcomplex $K \subset Y$ is called $\Gamma$-locally $\pi$-finite, if for every cell $c \subset K$ the subset $\{\gamma \in \Gamma \mid \gamma . c \subset K\} \subset \Gamma$ is a union of only finitely many right-translates of $\pi \subset \Gamma$.

Remark 5.3.2. In the following we always assume that $\Gamma$ acts properly on $Y$. In this case the definition is saying that given any $\Gamma$-cell of $Y$ the subcomplex $K$ may contain only finitely many of the $\pi$-cells that together form the $\Gamma$-cell. Equivalently, $K \subset Y$ is $\Gamma$-locally $\pi$-finite iff for every $\Gamma$-compact subspace $X \subset Y$ the space $K \cap X$ is $\pi$-compact.

If, however, the $\Gamma$-action is not proper, then $\pi$-finiteness is determined by looking at the group $\Gamma$, not at the complex $K$. For example, when $Y$ is compact, then $K$ can only be $\Gamma$-locally $\pi$-finite if $K$ is empty or $|\Gamma: \pi|<\infty$.

The reason why the above definition is made this way is to ensure that the preimage of a $\Gamma$-locally $\pi$-finite subcomplex under a $\Gamma$-equivariant map is still $\Gamma$-locally $\pi$-finite.

If $K \subset Y$ is a subcomplex of a CW-complex, we will in the following denote by $Y \backslash K$ the sub-CW-complex that is the closure of the complement, and by $\partial K$ the subcomplex $K \cap(Y \backslash K)$.

Definition 5.3.3. We say that a generalized equivariant homology theory $E$ has $l$-restrictions if for every inclusion of groups $\pi \subset \Gamma$, for every proper $\Gamma$ $\mathrm{CW}$-complex $Y$ and for every $\Gamma$-locally $\pi$-finite subcomplex $K \subset Y$ there is a natural homomorphism $r_{K}: E_{*}^{\Gamma}(Y) \rightarrow E_{*}^{\pi}(Y, Y \backslash K) \cong E_{*}^{\pi}(K, \partial K)$.

Furthermore, we require these maps to be compatible with the induction isomorphisms in the following sense: When $\Gamma$ acts freely on $Y$ and $U_{K}$ is a $\pi$-invariant neighborhood of $K$ such that $\gamma . U_{K} \cap U_{K} \neq \emptyset$ only for $\gamma \in \pi \subset \Gamma$, then there are automatically induction isomorphisms

$$
E_{*}^{\Gamma}(Y) \cong E_{*}(Y / \Gamma), \quad E_{*}^{\pi}(K, \partial K) \cong E_{*}(K / \pi, \partial K / \pi) .
$$

There is also the usual forgetful map

$$
E_{*}(Y / \Gamma) \rightarrow E_{*}(Y / \Gamma, Y / \Gamma \backslash K / \pi) \cong E_{*}(K / \pi, \partial K / \pi) .
$$

We require that the following diagram commutes:

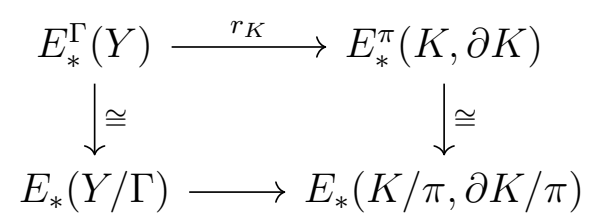


Lemma 5.3.4. K-homology has lf-restrictions.

Proof. As in Section 4.7 we use the geometric picture of equivariant K-homology. Let $\pi, \Gamma, Y, K$ as before. And let $(M, s, E, f)$ be a singular $\Gamma$-Spin manifold with a $\Gamma$-vector bundle, representing a K-homology class in $K O_{*}^{\Gamma}(Y)$.

To define the lf-restriction map we have to find any $\pi$-invariant $\pi$-compact submanifold with boundary $M^{\prime} \subset M$, such that $f\left(M \backslash M^{\prime}\right) \subset Y \backslash K$. The restricted K-homology cycle $\left(M^{\prime}, s_{\mid M^{\prime}}, E_{\mid M^{\prime}}, f_{M^{\prime}}\right)$, with an action of only $\pi$, then defines an element in $K O_{*}^{\pi}(Y, Y \backslash K) \cong K O_{*}^{\pi}(K, \partial K)$ that does not depend on the concrete choice of $M^{\prime}$. The construction is compatible with taking disjoint unions, with vector bundle addition and with vector bundle modification. Because the method given below to find $M^{\prime}$ can also be applied to bordisms, the construction is compatible with the bordism relation and therefore defines a natural group homomorphism from $K O_{*}^{\Gamma}(Y)$ to $K O_{*}^{\pi}(K, \partial K)$.

If $\Gamma$ acts freely on $Y$ and $U_{K} / \Gamma=U_{K} / \pi$ for a $\pi$-invariant neighborhood of $K$, then it also holds that $\Gamma$ acts freely on $M$ and $V_{K} / \Gamma=V_{K} / \pi$ for a $\pi$-invariant neighborhood $V_{K}$ of $f^{-1}(K)$. We may arrange that $M^{\prime} \subset V_{K}$. The induction isomorphisms are given by quotienting the group action out of a given K-homology cycle. Therefore, we can see on the level of cycles that the diagram of Definition 5.3.3 is commutative.

It remains to construct the submanifold $M^{\prime} \subset M$. Because $M$ is smooth and $\Gamma \curvearrowright M$ acts properly, $M$ allows a $\Gamma$-CW-structure ([Il100]) and we can equivariantly homotope the map $f: M \rightarrow Y$ to a cellular map. By Remark 5.3.2 the preimage $f^{-1}(K)$ is $\Gamma$-locally $\pi$-finite in $M$. Because $M$ is $\Gamma$-compact, $f^{-1}(K)$ is $\pi$-compact. Now we choose a $\Gamma$-invariant Riemannian metric on $M$ and consider the function $d: M \rightarrow \mathbb{R}$ that assigns to $m \in M$ the distance from $m$ to $f^{-1}(K)$. This function is $\pi$-equivariant. The induced function $\bar{d}: M / \pi \rightarrow \mathbb{R}$ is the distance function (in the metric induced from $M$ ) to the compact set $f^{-1}(K) / \pi$. Hence $\bar{d}$ is proper.

Next, we need an equivariant smooth approximation of $d$. Because the action $\Gamma \curvearrowright M$ is proper, every point $m \in M$ has a $\Gamma$-invariant neighborhood of the form $\bigsqcup_{[\gamma] \in \Gamma / \operatorname{Stab}(m)} W_{m,[\gamma]}$, where $\operatorname{Stab}(m)$ is the stabilizer of $m$. Because $M$ is $\Gamma$-compact, it is covered by a finite set of such neighborhoods. Because all stabilizer groups are finite, one can construct a $\Gamma$-invariant smooth partition of unity subordinate to the covering. Using the partition of unity we can now smoothen $d$ separately on each neighborhood. This works by choosing a smoothening on one $W_{m,[\gamma]}$, averaging it over the finite stabilizer group and extending it equivariantly to the whole neighborhood.

Finally, pick any regular value $r>0$ of $d$ such that $d^{-1}([r, \infty)) \cap f^{-1}(K)=$ $\emptyset$. The preimage $M^{\prime}=d^{-1}((-\infty, r]) \subset M$ is a $\pi$-invariant submanifold with boundary. Because $\bar{d}$ is still a proper function after smooth approximation, $M^{\prime}$ is $\pi$-compact.

Remark 5.3.5. In the above construction we could have included an intermediate step where we consider the original K-homology cycle $(M, s, E, f)$ with 
only a $\pi$-action and think of it as representing a class in some sort of locally finite K-homology (this is the motivation for the name "lf-restrictions"). From a conceptual viewpoint it would be best to formulate axioms for a locally finite equivariant generalized homology theory, from which the lf-restrictions could be obtained as a special case. But for the moment we are mostly interested in applying the lf-restrictions to extend the transfer map.

Now we use lf-restrictions to construct the extension of the transfer map.

Theorem 5.3.6. Let $M$ be a closed connected smooth manifold and let $N$ be a codimension-2 closed connected submanifold with trivialized normal bundle. Assume that $\pi_{1}(N) \rightarrow \pi_{1}(M)$ is injective and $\pi_{2}(N) \rightarrow \pi_{2}(M)$ is surjective.

Then there is, for any generalized multiplicative cohomology theory $E$ that has lf-restrictions, a map

$$
\operatorname{tr}_{\pi_{1}(M)}: E_{*}\left(\mathbf{B} \pi_{1}(M)\right) \rightarrow E_{*-2}\left(\mathbf{B} \pi_{1}(N)\right)
$$

such that the following diagram commutes:

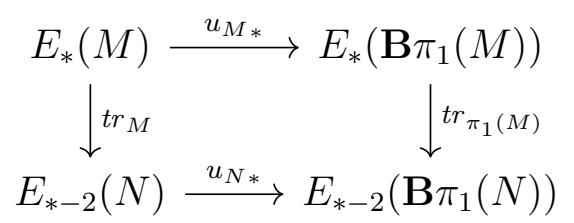

Moreover, the transfer $\operatorname{tr}_{\pi_{1}(M)}$ is natural for multiplicative transformations of equivariant cohomology theories with lf-restrictions.

Proof. As before let $D \nu$ be the disk bundle inside the normal bundle of $N$. The transfer map $\operatorname{tr}_{M}: E_{*}(M) \rightarrow E_{*-2}(N)$ is given by passing to the relative group $E_{*}(M, M \backslash D \nu) \cong E_{*}(D \nu, \partial D \nu)$ and then taking the cap product with the Thom class $f^{*}(e) \in E^{2}(D \nu, \partial D \nu)$, which is the pullback of the suspended unit $e \in E^{2}\left(D^{2}, S^{1}\right)$ under a map $f:(D \nu, \partial D \nu) \rightarrow\left(D^{2}, S^{1}\right)$ representing the trivialization of the normal bundle.

Using the equivariant picture the same map can also be expressed as follows: Let $\bar{M}$ be the covering of $M$ corresponding to the subgroup $\pi_{1}(N) \subset \pi_{1}(M)$ and let $N \subset \bar{M}$ be a lift of the inclusion $N \subset M$.

Then $\pi_{1}(N) \rightarrow \pi_{1}(\bar{M})$ is an isomorphism and $\pi_{2}(N) \rightarrow \pi_{2}(\bar{M})$ is surjective. From the long exact sequence of homotopy groups it follows $\pi_{k}(\bar{M}, D \nu)=0$ for $k \leq 2$, and by the relative Hurewicz theorem the same is then true for $H_{k}(\bar{M}, D \nu) \cong H_{k}(\bar{M} \backslash D \nu, \partial D \nu)$. But now the long exact sequence of the pair $(\bar{M} \backslash D \nu, \partial D \nu)$ shows that the map $H_{1}(\partial D \nu ; \mathbb{Z}) \rightarrow H_{1}(\bar{M} \backslash D \nu ; \mathbb{Z})$ is an isomorphism. And by the Universal Coefficient Theorem the same is then true for the map $H^{1}(\bar{M} \backslash D \nu ; \mathbb{Z}) \rightarrow H^{1}(\partial D \nu ; \mathbb{Z})$.

Since $S^{1}$ is the Eilenberg-MacLane space $K(\mathbb{Z}, 1)$, this means that the trivialization map $(D \nu, \partial D \nu) \rightarrow\left(D^{2}, S^{1}\right)$ of the normal bundle can be extended to a map $\bar{f}:(\bar{M}, \bar{M} \backslash D \nu) \rightarrow\left(D^{2}, S^{1}\right)$, and the extension is unique up to 
homotopy. Precomposition with the projection of the universal covering $\widetilde{M} \rightarrow$ $\bar{M}$ gives a $\pi_{1}(N)$-invariant map $\widetilde{f}:(\widetilde{M}, \widetilde{M} \backslash \widetilde{D \nu}) \rightarrow\left(D^{2}, S^{1}\right)$.

Let now $r_{\widetilde{D \nu}}: E_{*}^{\pi_{1}(M)}(\widetilde{M}) \rightarrow E_{*}^{\pi_{1}(N)}(\widetilde{D \nu}, \partial \widetilde{D \nu})$ be the lf-restriction associated to the inclusion of groups $\pi_{1}(N) \subset \pi_{1}(M)$ and the $\pi_{1}(M)$-locally $\pi_{1}(N)$ compact subspace $\widetilde{D \nu} \subset \widetilde{M}$. And let $\widetilde{f}^{*}(e) \in E^{2}(\widetilde{M}, \widetilde{M} \backslash \widetilde{D \nu}) \cong E^{2}(\widetilde{D \nu}, \partial \widetilde{D \nu})$ be the pullback of the doubly suspended unit $e \in E^{2}\left(D^{2}, S^{1}\right)$.

Then there is a map

$$
\widetilde{\operatorname{tr}}_{M}: E_{*}^{\pi_{1}(M)}(\widetilde{M}) \rightarrow E_{*}^{\pi_{1}(N)}(\widetilde{D \nu}, \partial \widetilde{D \nu}) \rightarrow E_{*-2}^{\pi_{1}(N)}(\widetilde{D \nu})
$$

given by $\widetilde{t r}_{M}(x)=r_{\widetilde{D \nu}}(x) \cap \widetilde{f}^{*}(e)$. Via the induction isomorphisms this corresponds to a map $E_{*}(M) \rightarrow E_{*-2}(N)$. Because the lf-restriction is compatible with induction isomorphisms, this map coincides with the transfer map $\operatorname{tr}_{M}$.

Next, let $\widetilde{u}_{M}: \widetilde{M} \rightarrow \mathbf{E} \pi_{1}(M)$ be a classifying map. We will construct a $\pi_{1}(N)$-invariant map $\hat{f}:\left(\mathbf{E} \pi_{1}(M), \mathbf{E} \pi_{1}(M) \backslash K\right) \rightarrow\left(D^{2}, S^{1}\right)$ such that the subspace $K \subset \mathbf{E} \pi_{1}(M)$ is $\pi_{1}(M)$-locally $\pi_{1}(N)$-compact, $\widetilde{u}_{M}$ becomes a map of pairs $(\widetilde{M}, \widetilde{M} \backslash \widetilde{D \nu}) \rightarrow\left(\mathbf{E} \pi_{1}(M), \mathbf{E} \pi_{1}(M) \backslash K\right)$ and $\hat{f} \circ \widetilde{u}_{M}$ is homotopic to $\widetilde{f}$ as a map of pairs.

Then, if $i_{K}: K \rightarrow \mathbf{E} \pi_{1}(M)$ and $r_{K}: E_{*}^{\pi_{1}(M)}\left(\mathbf{E} \pi_{1}(M)\right) \rightarrow E_{*}^{\pi_{1}(N)}(K, \partial K)$ are the inclusion and the lf-restriction, there is a map

$$
\widetilde{\operatorname{tr}}_{\pi_{1}(M)}: E_{*}^{\pi_{1}(M)}\left(\mathbf{E} \pi_{1}(M)\right) \rightarrow E_{*-2}^{\pi_{1}(N)}\left(\mathbf{E} \pi_{1}(M)\right)
$$

given by $\widetilde{t r}_{\pi_{1}(M)}(x)=\left(i_{K}\right)_{*}\left(r_{K}(x) \cap \hat{f}^{*}(e)\right)$.

But $\mathbf{E} \pi_{1}(M)$ is also a model for $\mathbf{E} \pi_{1}(N)$ (and $\widetilde{N} \rightarrow \widetilde{M} \rightarrow \mathbf{E} \pi_{1}(M)$ a classifying map). Therefore, under the induction isomorphisms this gives a map $\operatorname{tr}_{\pi_{1}(M)}: E_{*}\left(\mathbf{B} \pi_{1}(M)\right) \rightarrow E_{*-2}\left(\mathbf{B} \pi_{1}(N)\right)$. The naturality of the capproduct implies $\widetilde{t r}_{\pi_{1}(M)} \circ\left(\widetilde{u}_{M}\right)_{*}=\left(\widetilde{u}_{N}\right)_{*} \circ \widetilde{t r}_{M}$ and therefore $\operatorname{tr}_{\pi_{1}(M)} \circ\left(u_{M}\right)_{*}=$ $\left(u_{N}\right)_{*} \circ t r_{\pi_{1}(M)}$. Furthermore, the transfer map $t r_{\pi_{1}(M)}$ only depends on the homotopy class of $\hat{f}$, and if for $\hat{f}$ fixed the set $K$ is made larger (within the allowed bounds), the transfer map will not change by naturality of the cap product.

It remains to find $\hat{f}$ and $K$. Note that $\mathbf{E} \pi_{1}(M)$ can be obtained from $\widetilde{M}$ by adding free $\pi_{1}(M)$-orbits of cells of dimension $\geq 3$. Starting with $\hat{f}_{0}=\widetilde{M}$ and $K_{0}=\widetilde{D \nu}$, we proceed inductively on the dimension of added cells, extending $\widetilde{f}$ and possibly extending $K$ in each step.

When a $\pi_{1}(M)$-orbit of $k$-cells is added, then by the induction hypothesis on local compactness of $K$ on the $(k-1)$-skeleton only finitely many of the $\pi_{1}(N)$-orbits inside it attach to cells lying in $K$. These finitely many $\pi_{1}(N)$ orbits have to be added to $K$. On the other $\pi_{1}(N)$-orbits the map $\widetilde{f}$ can be extended with values in $S^{1}$ because $\pi_{k}\left(S^{1}\right)=0$ for $k>1$. The resulting map $\hat{f}:\left(\mathbf{E} \pi_{1}(M), \mathbf{E} \pi_{1}(M) \backslash K\right) \rightarrow\left(D^{2}, S^{1}\right)$ is unique up to homotopy and enlarging of $K$. 
Remark 5.3.7. As mentioned in the proof, the transfer map $t r_{\pi_{1}(M)}$ does not depend on the choices made for $\hat{f}$ and $K$. Just like $t r_{M}$ it does, however, depend on the embedding $N \subset M$ and on the choice of the trivialization of the normal bundle $D \nu$.

Remark 5.3.8. As in Theorem 5.1.1 the assumption that $\pi_{2}(N) \rightarrow \pi_{2}(M)$ be surjective can be weakened to $\pi_{2}(N) \oplus \pi_{2}(M \backslash N) \rightarrow \pi_{2}(M)$ surjective.

It is a natural question whether the extension of the transfer map could also be defined in the non-equivariant setting, i.e., using only the base spaces $\mathbf{B} \pi_{1}(M), \mathbf{B} \pi_{1}(N), \mathbf{B} \pi_{1}(M \backslash N)$ of the classifying spaces. This works if the pushout diagram of the fundamental groups induces a pushout diagram of the corresponding classifying spaces:

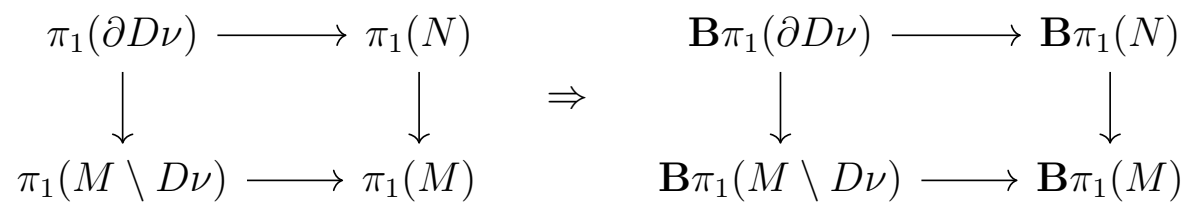

In this case the classifying map $\partial D \nu \rightarrow \mathbf{B} \pi_{1}(\partial D \nu)=\mathbf{B} \pi_{1}(N) \times S^{1}$ can be extended to classifying maps $N \rightarrow \mathbf{B} \pi_{1}(N) \times D^{2}$ and $M \backslash D \nu \rightarrow \mathbf{B} \pi_{1}(M \backslash D \nu)$ and these maps combine to a classifying map $M \rightarrow \mathbf{B} \pi_{1}(M)$ that respects the decomposition. Because the classifying maps induce isomorphisms of fundamental groups and $S^{1}=K(\mathbb{Z}, 1)$, the trivialization map $(D \nu, \partial D \nu) \rightarrow\left(D^{2}, S^{1}\right)$ extends to a map $g:\left(\mathbf{B} \pi_{1}(N) \times D^{2}, \mathbf{B} \pi_{1}(N) \times S^{1}\right) \rightarrow\left(D^{2}, S^{1}\right)$ (uniquely up to homotopy). Taking the cap product with $g^{*}(e)$ defines a transfer map

$$
E_{*}\left(\mathbf{B} \pi_{1}(M)\right) \rightarrow E_{*}\left(\mathbf{B} \pi_{1}(N) \times D^{2}, \mathbf{B} \pi_{1}(N) \times S^{1}\right) \rightarrow E_{*-2}\left(\mathbf{B} \pi_{1}(N) \times D^{2}\right)
$$

To see that this map coincides with the transfer constructed in Theorem 5.3.6, note that the construction in the first part of the proof can be applied to $\mathbf{B} \pi_{1}(N) \times D^{2} \subset \mathbf{B} \pi_{1}(M)$.

The resulting map $\tilde{f}:\left(\mathbf{E} \pi_{1}(M), \mathbf{E} \pi_{1}(M) \backslash \mathbf{E} \pi_{1}(N) \times D^{2}\right) \rightarrow\left(D^{2}, S^{1}\right)$ is a suitable candidate for $\hat{f}:\left(\mathbf{E} \pi_{1}(M), \mathbf{E} \pi_{1}(M) \backslash K\right) \rightarrow\left(D^{2}, S^{1}\right)$. Then, by uniqueness of $(\hat{f}, K)$, the two transfer maps are equal.

In fact, this non-equivariant approach can be applied to both classes of examples given in Section 5.2.

Lemma 5.3.9. In both Example 5.2.1 and Example 5.2.2 it is true that

$$
\mathbf{B} \pi_{1}(N) \times D^{2} \cup_{\mathbf{B} \pi_{1}(N) \times S^{1}} \mathbf{B} \pi_{1}(M \backslash N)
$$

is a model for $\mathbf{B} \pi_{1}(M)$.

Proof. For Example 5.2.1 - the embedding of $S^{1}$ into an oriented 3-manifold - we simply show that the spaces $N, \partial D \nu, M$ and $M \backslash D \nu$ are all aspherical. This is obvious for $N$ and $\partial D \nu$. Furthermore, since $\pi_{1}(M)$ is infinite, $\widetilde{M}$ is 
non-compact and therefore $H_{3}(\widetilde{M} ; \mathbb{Z})=0$. Also, $\pi_{2}(\widetilde{M})=0$ by assumption. Therefore $\widetilde{M}$ is contractible by the Hurewicz Theorem. It just remains $M \backslash D \nu$.

Because $M$ is aspherical, the Sphere Theorem implies that $M$ is an irreducible and hence a prime 3-manifold (see for example [Hat]). This means that $\pi_{2}(M \backslash N)=0$. Otherwise, the Sphere Theorem would give an embedded sphere $S^{2} \rightarrow M \backslash N \subset M$ representing a non-trivial element of $\pi_{2}(M \backslash N)$. This sphere would determine a decomposition, which would be trivial since $M$ is prime: $M=M^{\prime} \# D^{3}$. Then $N$ has to be contained in one of the two summands, and since $\pi_{1}(N) \rightarrow \pi_{1}(M)$ is injective, $N \subset M^{\prime}$. But now the embedded sphere has a null-homotopy inside the $D^{3}$-summand, which lies in $M \subset N$, giving a contradiction.

In addition to $\pi_{2}(M \backslash N)=0, H_{3}(\widetilde{M \backslash N} ; \mathbb{Z})=0$ holds as before, and from the Hurewicz Theorem it follows that $M \backslash N$ is aspherical.

For Example 5.2.2 assume that $N \rightarrow M \rightarrow \Sigma$ is a fiber bundle over an aspherical surface. Then $\pi_{1}(M)$ is an extension of $\pi_{1}(\Sigma)$ with kernel $\pi_{1}(N)$. The induced map $\mathbf{B} \pi_{1}(M) \rightarrow \mathbf{B} \pi_{1}(\Sigma)=\Sigma$ can be turned into a fiber bundle by passing to the model $\mathbf{E} \pi_{1}(M) \simeq \mathbf{E} \pi_{1}(M) \times \mathbf{E} \pi_{1}(\Sigma)$ with the diagonal action of $\pi_{1}(M)$ and $\pi: \mathbf{B} \pi_{1}(M) \rightarrow \mathbf{B} \pi_{1}(\Sigma)=\Sigma$ induced by the projection onto the second factor of the new model.

But now $\mathbf{B} \pi_{1}(M)$ decomposes as $\mathbf{B} \pi_{1}(M)=\pi^{-1}\left(D^{2} \subset \Sigma\right) \cup \pi^{-1}\left(\Sigma \backslash D^{2}\right)$. The pieces $\pi^{-1}\left(D^{2} \subset \Sigma\right), \pi^{-1}\left(\Sigma \backslash D^{2}\right)$ and the intersection $\pi^{-1}\left(\partial D^{2} \subset \Sigma\right)$ are classifying spaces for the groups $\pi_{1}(N), \pi_{1}(M \backslash N)$ and $\pi_{1}\left(N \times S^{1}\right)$, respectively.

On the other hand, the conditions on $\pi_{1}(N) \rightarrow \pi_{1}(M)$ and $\pi_{2}(N) \rightarrow \pi_{2}(M)$ do not imply that the classifying spaces always fit into a pushout diagram. The fundamental group $G$ occurring in the following example was given in Hil02, Section 14.10] as an example for a high-dimensional knot group that is not a 2-knot group. The space $\mathbf{B} \pi_{1}(G)$ was first constructed in [CS76] as an example for a "fake projective space".

Example 5.3.10. Consider the group $G=\mathbb{Z}^{3} \rtimes_{B} \mathbb{Z}$ where $\mathbb{Z}$ acts on $\mathbb{Z}^{3}$ by the matrix

$$
B=\left(\begin{array}{ccc}
0 & 1 & 0 \\
0 & 0 & 1 \\
-1 & 1 & 0
\end{array}\right) \text {. }
$$

Because the matrix $(B-1)$ is invertible, $G$ is normally generated by the generator of $\mathbb{Z}$. It fits into the following pushout diagram:

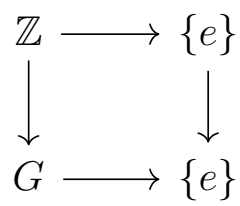

The homology of $G$ can be computed just like in Section 4.5 with the Wang sequence 


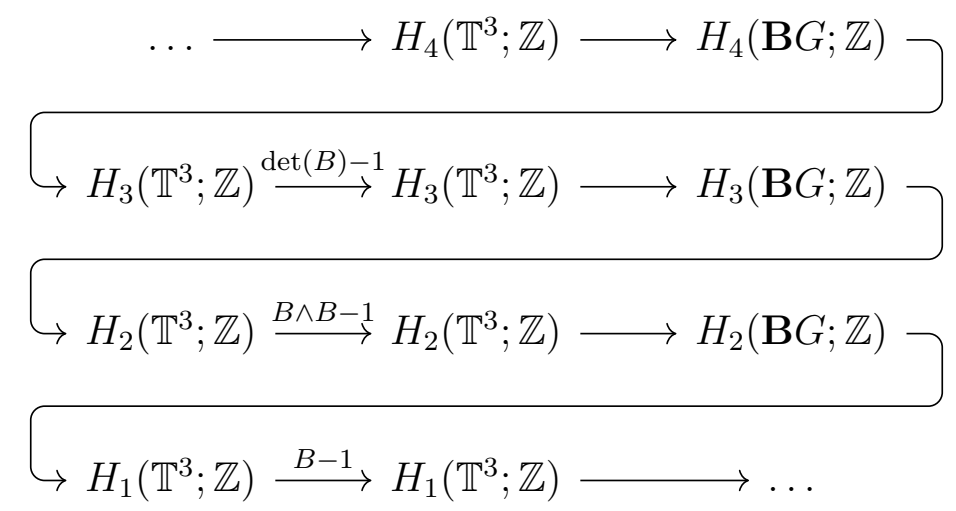

Using the basis $\left\{e_{1} \wedge e_{2}, e_{1} \wedge e_{3}, e_{2} \wedge e_{3}\right\}$ for $\mathbb{Z}^{3} \wedge \mathbb{Z}^{3}$ we calculate that the matrices

$$
B \wedge B-1=\left(\begin{array}{ccc}
-1 & 0 & 1 \\
1 & -1 & 0 \\
0 & 1 & -2
\end{array}\right) \quad B-1=\left(\begin{array}{ccc}
-1 & 1 & 0 \\
0 & -1 & 1 \\
-1 & 1 & -1
\end{array}\right)
$$

are both invertible. Also, $\operatorname{det}(B)-1=-2$. Hence

$$
H_{0}(\mathbf{B} G ; \mathbb{Z})=\mathbb{Z} ; \quad H_{1}(\mathbf{B} G ; \mathbb{Z})=0 ; \quad H_{2}(\mathbf{B} G ; \mathbb{Z})=0 ; \quad H_{3}(\mathbf{B} G ; \mathbb{Z})=\mathbb{Z}_{2} .
$$

In particular, this shows that in the pushout diagram

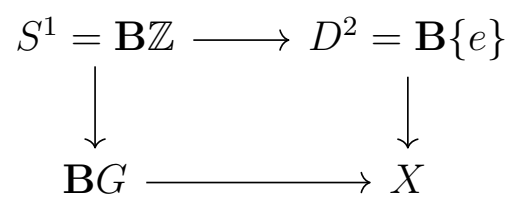

the space $X$ has a non-trivial third homology group and hence cannot be a classifying space for the trivial group.

To see that the pushout diagram of fundamental groups can occur in a situation where the transfer map exists, construct a high-dimensional manifold with fundamental group $G$. Perform 1-surgery on the generator of $\mathbb{Z} \subset G$ that normally generates $G$. Let $M$ be the resulting manifold and let $N \rightarrow M$ be the inclusion of the transversal sphere of the 1-surgery.

Remark 5.3.11. Even though the classifying spaces of the previous example do not fit into a pushout diagram, it is not clear if one can pass from $\pi_{1}(M \backslash D \nu)$ to a quotient, such that the classifying spaces of the resulting groups do fit into a pushout diagram.

More generally, disregarding the group $\pi_{1}(M \backslash D \nu)$, one could try to embed $\mathbf{B} \pi_{1}(N) \times D^{2}$ into a model of $\mathbf{B} \pi_{1}(M)$ such that

$$
H^{1}\left(\mathbf{B} \pi_{1}(M) \backslash \mathbf{B} \pi_{1}(N) \times D^{2} ; \mathbb{Z}\right) \rightarrow H^{1}\left(\mathbf{B} \pi_{1}(N) \times S^{1} ; \mathbb{Z}\right)
$$

is an isomorphism, and try to find a map $M \rightarrow \mathbf{B} \pi_{1}(M)$ restricting to maps $N \rightarrow \mathbf{B} \pi_{1}(N)$ and $M \backslash D \nu \rightarrow \mathbf{B} \pi_{1}(M) \backslash \mathbf{B} \pi_{1}(N) \times D^{2}$. It is not clear if this is possible. 


\subsection{Extending the transfer map further to $\underline{\mathrm{E}} G$}

In this section we extend the transfer map from $\mathbf{E} \pi_{1}(M)$ to the classifying space for proper actions $\mathbf{E} \pi_{1}(M)$. The basic idea is the same as in the proof of Theorem 5.3.6. To describe the transfer map in terms of a map into the pair of spaces $\left(D^{2}, S^{1}\right)$ and then to extend this map. Just as with the free classifying space $\mathbf{E} \Gamma$ we can, for every subgroup $\pi \subset \Gamma$, use $\underline{\mathbf{E}} \Gamma$ as a model for $\underline{\mathbf{E}} \pi$.

Definition 5.4.1. Let $\Gamma$ be a countable discrete group, $\pi$ a subgroup and $X$ a $\Gamma$-CW-complex.

A reference map on $X$ is a $\pi$-invariant cellular map of pairs

$$
(X, X \backslash K) \rightarrow\left(D^{2}, S^{1}\right)
$$

where $K \subset X$ is a $\Gamma$-locally $\pi$-finite subcomplex.

Two reference maps $f_{i}:\left(X, X \backslash K_{i}\right) \rightarrow\left(D^{2}, S^{1}\right), i \in\{0,1\}$ are equivalent if $f_{0}=f_{1}$ as maps from $X$ to $D^{2}$.

Two reference maps $f_{0}, f_{1}$ on $X$ are homotopic if there is a reference map $F$ on $X \times[0,1]$, such that the restrictions to $X \times\{0\}$ and $X \times\{1\}$ are equivalent to $f_{0}$ and $f_{1}$.

\section{Remark 5.4.2.}

1. If $(f, K)$ is a reference map on $X$ and $\phi: Y \rightarrow X$ is a morphism of $\Gamma$-CW complexes, then the pullback $\left(f \circ \phi, \phi^{-1}(K)\right)$ defines a reference map on $Y$.

2. The requirement that the homotopy $F$ must be a reference map itself means that "most" cells in $X$ must be mapped to $S^{1} \subset D^{2}$ during the entire homotopy.

A reference map $(f, K)$ on a $\Gamma$-CW-complex $X$ gives rise to a transfer homomorphism

$$
\operatorname{tr}_{f}: E_{*}^{\Gamma}(X) \rightarrow E_{*-2}^{\pi}(X), \quad x \mapsto\left(i_{K}\right)_{*}\left(r_{K}(x) \cap f^{*}(e)\right) .
$$

Here $i_{K}$ is the inclusion $K \rightarrow X, r_{K}$ is the lf-restriction $E_{*}^{\Gamma}(X) \rightarrow E_{*}^{\pi}(K, \partial K)$ and $e$ is the twice-suspended unit of the cohomology theory.

Equivalent reference maps give rise to the same transfer homomorphism. Indeed, for $K_{1} \subset K_{2}$ this is true by naturality of the cap product. And for general $K_{1}, K_{2}$ we note that $K_{1} \cup K_{2}$ is also a $\Gamma$-locally $\pi$-finite subcomplex. Furthermore, homotopic reference maps give rise to the same transfer map. Finally, if $(f, K)$ is a reference map on $X$ and $\phi: Y \rightarrow X$ is a morphism of $\Gamma$-CW complexes, then $\phi_{*} \circ t r_{f \circ \phi}=t r_{f} \circ \phi_{*}$. To extend the transfer map constructed in Theorem 5.3.6 further to $E_{*}^{\pi_{1}(M)}\left(\underline{\mathbf{E}} \pi_{1}(M)\right)$ it is therefore sufficient to find a lift (up to homotopy of reference maps) of the reference map $\hat{f}$ that induces the transfer map.

Next, we rephrase reference maps in terms of cohomology: 
Definition 5.4.3. Let $\Gamma$ be a countable discrete group and $\pi$ a subgroup.

A $\pi$-invariant cellular cochain with values in $\mathbb{Z}$ on a $\Gamma$-CW-complex is called a $\Gamma$ - $\pi$-almost-cocycle if its coboundary is non-zero only on $\Gamma$-locally $\pi$-finitely many cells.

Two $\Gamma$ - $\pi$-almost-cocycles $x_{0}, x_{1}$ are cohomologous if there is a $\pi$-invariant 1-chain $y$, such that $x_{1}-x_{0}=\partial y$ outside of a $\Gamma$-locally $\pi$-finite subcomplex. In this case we write $x_{1}-x_{0} \approx \partial y$.

Lemma 5.4.4. There is a correspondence between homotopy classes of reference maps and $\Gamma$ - $\pi$-almost-cohomology classes in degree 1.

Proof. A reference map $f$ gives rise to a $\Gamma$ - $\pi$-almost-cocycle by assigning to a 1-cell $c$ the value $f_{\mid c}{ }^{*}(1)$ with $1 \in H^{1}\left(S^{1} ; \mathbb{Z}\right)$ a fixed generator. Conversely, if a $\Gamma$ - $\pi$-almost-cocycle $x$ is given, the requirement $f_{\mid c}{ }^{*}(1)=x(c)$ defines a (unique up to homotopy) map $f$ on the 1-skeleton. By the almost-cocycle-property of $x$ this map can be extended to a reference map on the 2-skeleton. And because $S^{1}$ is aspherical, it can be extended further to all of $X$. The extensions are unique up to homotopy (of reference maps), because $S^{1}$ is aspherical.

Similarly, if $x_{f_{0}}, x_{f_{1}}$ are the cocycles corresponding to two reference maps, then a homotopy $F$ between these reference maps determines a 1-almostcocycle on $X \times[0,1]$, which in turn gives a 0 -cochain $y$ such that $x_{f_{1}}-x_{f_{0}} \approx \partial y$. In the other direction, if $x_{1}-x_{0} \approx \partial y$, then $x_{0}, x_{1}$ and $y$ together define a 1 -almost-cocycle on $X \times[0,1]$ which in turn gives a reference map on $X \times[0,1]$.

We now want to show that up to homotopy every reference map on the classifying space $\mathbf{E} \Gamma$ can be lifted to the proper classifying space $\underline{\mathbf{E}} \Gamma$. By the correspondence this amounts to lifting a $\Gamma$ - $\pi$-almost-cocycle.

The lifting problem does not depend on the concrete models for $\mathbf{E} \Gamma, \underline{\mathbf{E}} \Gamma$ because two different models are always $\Gamma$-homotopy equivalent, and the $\Gamma$ homotopies give rise to homotopies of reference maps.

For $\underline{\mathbf{E}} \Gamma$ we take any $\Gamma$-CW-complex model that is locally compact. For example, such a model can be obtained by starting with the simplicial model described in Mislin's appendix to [Val02], where $n$-simplices correspond to finite subsets of $\Gamma$ of cardinality $n+1$. After passing to the barycentric subdivision this becomes a $\Gamma$-CW-complex with countably many $\Gamma$-cells. We fix an enumeration of the $\Gamma$-cells, such that every $n$-cell occurs in the enumeration after the $(\leq n)$-cells it is attached to. Then we build the iterated mapping cylinder of the (countably infinite) chain of inclusions $\emptyset=F_{0} \subset F_{1} \subset F_{2} \subset \ldots$, where $F_{k}$ is obtained from $F_{k-1}$ by adding the $k$-th $\Gamma$-cell in the enumeration. Because all stabilizers are finite, the iterated mapping cylinder of all finite chains of inclusions $F_{0} \subset \cdots \subset F_{k}$ is locally compact (as a simple CW-complex without group action) and the same then holds for the whole iterated mapping cylinder.

For $\mathbf{E} \Gamma$ we also start with a locally compact $\Gamma$-CW-complex model $\mathbf{E} \Gamma_{0}$ that we similarly obtain from any $\Gamma$-CW-complex model with countably many cells. 
Then we set $\mathbf{E} \Gamma=\mathbf{E} \Gamma_{0} \times \underline{\mathbf{E}} \Gamma$ with the diagonal $\Gamma$-action and the canonical map $\widetilde{q}: \mathbf{E} \Gamma \rightarrow \underline{\mathbf{E}} \Gamma$ becomes the projection onto the second factor. The quotient spaces $\pi \backslash \mathbf{E} \Gamma$ and $\Gamma \backslash \underline{\mathbf{E}} \Gamma$ are locally compact CW-complexes. Since our coefficient module $\mathbb{Z}$ has the trivial $\Gamma$-action, $H_{\pi}^{*}(\mathbf{E} \Gamma ; \mathbb{Z})=H^{*}(\pi \backslash \mathbf{E} \Gamma ; \mathbb{Z})$ and $H_{\pi}^{*}(\underline{\mathbf{E}} \Gamma ; \mathbb{Z})=H^{*}(\pi \backslash \underline{\mathbf{E}} \Gamma ; \mathbb{Z})$ (here $H_{\pi}^{*}$ is the Bredon equivariant cohomology).

The map $q: \pi \backslash \mathbf{E} \Gamma \rightarrow \pi \backslash \underline{\mathbf{E}} \Gamma$ is not a fibration, but with sheaf cohomology there is still the Leray spectral sequence. Using the terminology of Bredon [Bre67] we let the "families of supports" $\Psi$ and $\Phi$ be the families of compact sets on $\pi \backslash \mathbf{E} \Gamma$ and $\pi \backslash \underline{\mathbf{E}} \Gamma$ respectively. Then both $\Psi$ and $\Phi$ are paracompactifying in the sense of [Bre67, I.6.1], which means that sheaf cohomology with supports in $\Psi, \Phi$ and constant coefficient module $\mathbb{Z}$ coincides with singular cohomology ([Bre67, III.1.1]). By [Bre67, II.10.5] all fibers $q^{-1}(y)$ are $\Psi$-taut. Then by [Bre67, IV.6.1] there is a spectral sequence $H^{p}\left(\pi \backslash \underline{\mathbf{E}} \Gamma ; \mathcal{A}^{r}\right) \Rightarrow H^{p+r}(\pi \backslash \mathbf{E} \Gamma ; \mathbb{Z})$ where the germ of the sheaf $\mathcal{A}^{r}$ at a point $y \in \pi \backslash \underline{\mathbf{E}} \Gamma$ is given by $H^{r}\left(q^{-1}(y) ; \mathbb{Z}\right)$. From the choice of the model for $\mathbf{E} \Gamma$ it is clear that the preimages $f^{-1}(y)$ are models for the classifying spaces $\mathbf{B} \pi_{y}$ of the stabilizer groups $\pi_{y} \subset \pi$.

Theorem 5.4.5. Let $\pi \subset \Gamma$ be finitely presented groups, $\mathrm{E} \Gamma$ and $\underline{\mathbf{E}} \Gamma$ be represented by the models above, and let $\widetilde{q}: \mathbf{E} \Gamma \rightarrow \underline{\mathbf{E}} \Gamma$ represent the canonical map. Then any $\Gamma-\pi$-almost-cocycle of degree 1 on $\mathbf{E} \Gamma$ can be lifted to a $\Gamma-\pi$ almost cocycle on $\underline{\mathrm{E}} \Gamma$. Furthermore, any two lifts of the same almost-cocycle are cohomologous as almost-cocycles.

Proof. Let a 1-almost-cocycle $\xi$ be given on $\mathbf{E} \Gamma$. We can remove $\Gamma$-locally $\pi$ finitely many cells from $\mathbf{E} \Gamma$ such that $\xi$ becomes a true $\pi$-invariant cocycle on the remaining $\mathrm{CW}$-complex $\mathbf{E} \Gamma \backslash K_{0}$. Indeed, we have to remove the $\Gamma$-locally $\pi$-finitely many 2 -cells where $\xi$ is not a cocycle, and then inductively all cells of higher dimension that attach to already removed cells. In each step we only have to remove $\Gamma$-locally $\pi$-finitely many cells, then this is also true in total.

Because $\widetilde{q}$ is $\Gamma$-invariant and the action of $\Gamma$ on both $\mathbf{E} \Gamma$ and $\mathbf{E} \Gamma$ is proper, the image of the removed set $\widetilde{q}(K)$ is $\Gamma$-locally $\pi$-finite. By Remark 5.3.2 the preimage of this set $K=q^{-1}\left(q\left(K_{0}\right)\right)$ is also $\Gamma$-locally $\pi$-finite. Since in our model $\widetilde{q}$ is just the projection onto the second factor, both $\mathbf{E} \Gamma \backslash K$ and $\underline{\mathbf{E}} \Gamma \backslash q(K)$ are $(\pi$-invariant) sub-CW-complexes of $\mathbf{E} \Gamma$ and $\underline{\mathbf{E}} \Gamma$, respectively.

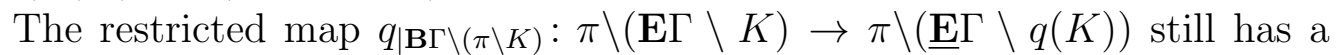
Leray spectral sequence as described above. Because the stabilizer groups $\pi_{y}$ are all finite, $H^{1}\left(\mathbf{B} \pi_{y} ; \mathbb{Z}\right)=0$. It follows that $E_{2}^{0,1}=0$ on the second page of the spectral sequence. On the $\infty$-page we then have $E_{\infty}^{0,1}=0$ and $E_{\infty}^{1,0}=E_{2}^{1,0}=H^{1}(\pi \backslash(\underline{\mathbf{E}} \Gamma \backslash q(K)) ; \mathbb{Z})$. This means that

$$
H^{1}(\pi \backslash(\mathbf{E} \Gamma \backslash K) ; \mathbb{Z}) \cong H^{1}(\pi \backslash(\underline{\mathbf{E}} \Gamma \backslash q(K)) ; \mathbb{Z})
$$

and the isomorphism is given by $q_{\mid \mathbf{B} \Gamma \backslash(\pi \backslash K)}$.

In particular, $\xi$ lifts to a $\pi$-invariant (true) cocycle on $\underline{\mathbf{E}} \Gamma \backslash q(K)$ which then defines an almost-cocycle on $\underline{\mathbf{E}} \Gamma$. And any two lifts are cohomologous. 
From the previous theorem, together with the description of the transfer map in terms of almost-cocycles, it follows:

Corollary 5.4.6. The transfer map can be extended to the classifying space for proper actions $\mathbf{E} \Gamma$ :

Let $M$ be a closed connected Spin manifold and $N$ a closed connected submanifold of codimension 2 with trivialized normal bundle. Assume that the induced map $\pi_{1}(N) \rightarrow \pi_{1}(M)$ is injective and $\pi_{2}(N) \rightarrow \pi_{2}(M)$ is surjective.

Then there is, for any generalized multiplicative equivariant cohomology theory E with lf-restrictions, a map

$$
\operatorname{tr} \pi_{\pi_{1}(M)}: E_{*}^{\pi_{1}(M)}\left(\underline{\mathbf{E}} \pi_{1}(M)\right) \rightarrow E_{*-2}^{\pi_{1}(N)}\left(\underline{\mathbf{E}} \pi_{1}(N)\right)
$$

such that the following diagram commutes:

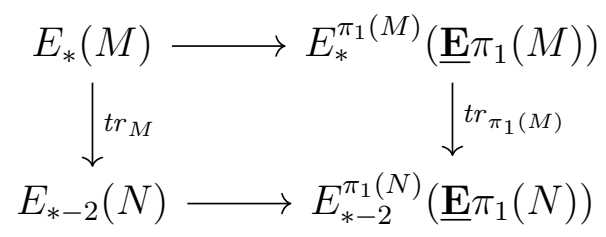

Moreover, the transfer $\operatorname{tr}_{\pi_{1}(M)}$ is natural for multiplicative transformations of cohomology theories with lf-restrictions.

Remark 5.4.7. Since the lift of the reference map is unique (up to homotopy), the extension of the transfer map should be canonical in some sense. Recall however that already the construction of Theorem 5.3.6 depends not only on the groups $\pi_{1}(N) \subset \pi_{1}(M)$, but also on the specific embedding $N \subset M$ and the trivialization of the normal bundle.

\section{Generalization to higher cohomology degrees}

By setting $\pi=\Gamma$ and not removing any cells it is clear that the proof of Theorem 5.4.5 also works to show that $\widetilde{q}$ induces an isomorphism $H_{\Gamma}^{1}(\underline{\mathbf{E}} \Gamma ; \mathbb{Z}) \cong$ $H_{\Gamma}^{1}(\mathbf{E} \Gamma ; \mathbb{Z})$.

The spectral sequence can be exploited further to give results in higher degrees. The next simplest step is to relate $H_{\Gamma}^{n}(\mathbf{E} \Gamma ; \mathbb{Z})$ to $H_{\Gamma}^{n}(\underline{\mathbf{E}} \Gamma ; \mathbb{Z})$ under the assumption that $H^{k}(\mathbf{B} G ; \mathbb{Z})=0$ for all finite subgroups $G \subset \Gamma$ and all $1 \leq k<n$.

In particular, for $n=2$ the assumption is automatically fulfilled. This case might also play a role in future work on the codimension- 2 transfer: If the normal bundle of $N \subset M$ is orientable but not trivializable, its twist is described by a second cohomology class. When we try to extend the transfer map, the information about the twist has to be carried around. Therefore it is natural to ask when an element $\xi \in H_{G}^{2}(\mathbf{E} G ; \mathbb{Z})$ can be lifted to $H_{G}^{2}(\underline{\mathbf{E}} G ; \mathbb{Z})$. The following theorem provides the answer: 
Theorem 5.4.8. Let $\Gamma$ be a countable discrete group and $q: \mathrm{B} \Gamma \rightarrow \Gamma \backslash \underline{\mathbf{E}} \Gamma$ the canonical map from the classifying space for free $\Gamma$-actions to the classifying space for proper $\Gamma$-actions. Assume that $n \geq 2$ and $H^{k}(\mathbf{B} G ; \mathbb{Z})=0$ for all finite subgroups $G \subset \Gamma$ and all $1 \leq k<n$.

Then $q$ induces an injection $q^{*}: H^{n}(\Gamma \backslash \underline{\mathbf{E}} \Gamma ; \mathbb{Z}) \rightarrow H^{n}(\mathbf{B} \Gamma ; \mathbb{Z})$. Furthermore, an element $\xi \in H^{n}(\mathbf{B} \Gamma ; \mathbb{Z})$ lies in the image of the induced map iff $i_{G}{ }^{*}(\xi)=$ $0 \in H^{n}(\mathbf{B} G ; \mathbb{Z})$ for all inclusions of finite subgroups $i_{G}: \mathbf{B} G \rightarrow \mathbf{B} \Gamma$.

Proof. We use the same spectral sequence as in Theorem 5.4.5. For the computation of $H^{n}(\mathbf{B} \Gamma ; \mathbb{Z})$ there are only two relevant (i.e., non-vanishing) entries on the $E_{\infty}$ page: One is $E_{\infty}^{n, 0}=E_{2}^{n, 0}=H_{\Gamma}^{n}(\underline{\mathbf{E}} \Gamma ; \mathbb{Z})$, the other one is $E_{\infty}^{0, n}$ which is a subgroup of $E_{2}^{0, n}$. Together they produce the extension problem

$$
0 \longrightarrow E_{\infty}^{n, 0} \longrightarrow H^{n}(\mathrm{~B} \Gamma ; \mathbb{Z}) \longrightarrow E_{\infty}^{0, n} \longrightarrow 0
$$

This shows that the induced map $q^{*}$ is injective.

If $\xi$ lies in the image of $q^{*}$ and $G \subset \Gamma$ is a finite subgroup, then the commutative diagram

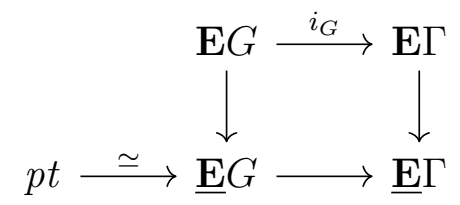

implies $i_{G}{ }^{*}(\xi)=0 \in H^{n}(\mathbf{B} G ; \mathbb{Z})$. Conversely, if $\xi$ is not in the image of $q^{*}$ then it maps to a non-trivial element of $E_{2}^{0, n}$. This group is by definition the group of global sections from $\Gamma \backslash \underline{\mathbf{E}} \Gamma$ into the sheaf $\mathcal{A}$ with germs $\mathcal{A}_{y}=H^{n}\left(\mathbf{B} \Gamma_{y} ; \mathbb{Z}\right)$. Since the image of $\xi$ is a non-trivial section, there is a point $y \in \Gamma \backslash \underline{\mathbf{E}} \Gamma$ such that the restriction of $\xi$ to $q^{-1}(y)$ is non-trivial. Recall now that $q^{-1}(y)$ is a model for $\mathbf{B} \Gamma_{y}$ and the inclusion of the fiber represents the canonical map $\mathbf{B} \Gamma_{y} \rightarrow \mathbf{B} \Gamma$. This finishes the proof.

Remark 5.4.9. With the same approach as in Theorem 5.4.5 this result can also be applied to almost-cohomology classes. 


\section{Bibliography}

[BD82] Paul Baum and Ronald G. Douglas. $K$ homology and index theory. In Operator algebras and applications, Part I (Kingston, Ont., 1980), volume 38 of Proc. Sympos. Pure Math., pages 117173. Amer. Math. Soc., Providence, R.I., 1982.

[BD10] Dmitry Bolotov and Alexander Dranishnikov. On Gromov's scalar curvature conjecture. Proc. Amer. Math. Soc., 138(4):1517-1524, 2010.

[BD14] Dmitry Bolotov and Alexander Dranishnikov. On Gromov's conjecture for totally non-spin manifolds. the unpublished second version, https://arxiv.org/abs/1402.4510, 2014.

[BD16] Dmitry Bolotov and Alexander Dranishnikov. On Gromov's conjecture for totally non-spin manifolds. J. Topol. Anal., 8(4):571587, 2016.

[Bes08] Arthur L. Besse. Einstein manifolds. Classics in Mathematics. Springer-Verlag, Berlin, 2008. Reprint of the 1987 edition.

[BHS07] Paul Baum, Nigel Higson, and Thomas Schick. On the equivalence of geometric and analytic K-homology. Pure Appl. Math. Q., 3(1, Special Issue: In honor of Robert D. MacPherson. Part 3):1-24, 2007.

[BHS10] Paul Baum, Nigel Higson, and Thomas Schick. A geometric description of equivariant $K$-homology for proper actions. In Quanta of maths, volume 11 of Clay Math. Proc., pages 1-22. Amer. Math. Soc., Providence, RI, 2010.

[Bla98] Bruce Blackadar. K-theory for operator algebras, volume 5 of Mathematical Sciences Research Institute Publications. Cambridge University Press, Cambridge, second edition, 1998.

[BOOSW10] Paul Baum, Hervé Oyono-Oyono, Thomas Schick, and Michael Walter. Equivariant geometric $K$-homology for compact Lie group actions. Abh. Math. Semin. Univ. Hambg., 80(2):149-173, 2010.

[Bre67] Glen E. Bredon. Sheaf theory. McGraw-Hill Book Co., New York-Toronto, Ont.-London, 1967.

[Bro82] Kenneth S. Brown. Cohomology of groups, volume 87 of Graduate Texts in Mathematics. Springer-Verlag, New York-Berlin, 1982. 
[CS76] Sylvain E. Cappell and Julius L. Shaneson. Some new fourmanifolds. Ann. of Math. (2), 104(1):61-72, 1976.

[DK01] James F. Davis and Paul Kirk. Lecture notes in algebraic topology, volume 35 of Graduate Studies in Mathematics. American Mathematical Society, Providence, RI, 2001.

[EL69] C. M. Edwards and J. T. Lewis. Twisted group algebras. I, II. Comm. Math. Phys., 13:119-130; ibid. 131-141, 1969.

[ELPW10] Siegfried Echterhoff, Wolfgang Lück, N. Christopher Phillips, and Samuel Walters. The structure of crossed products of irrational rotation algebras by finite subgroups of $\mathrm{SL}_{2}(\mathbb{Z})$. J. Reine Angew. Math., 639:173-221, 2010.

[Eng17] Alexander Engel. Wrong way maps in uniformly finite homology and homology of groups. to appear in J. Homotopy Relat. Struct., https://arxiv.org/abs/1602.03374, 2017.

[Fed70] Herbert Federer. The singular sets of area minimizing rectifiable currents with codimension one and of area minimizing flat chains modulo two with arbitrary codimension. Bull. Amer. Math. Soc., 76:767-771, 1970 .

[Fri00] Thomas Friedrich. Dirac operators in Riemannian geometry, volume 25 of Graduate Studies in Mathematics. American Mathematical Society, Providence, RI, 2000. Translated from the 1997 German original by Andreas Nestke.

[GL80] Mikhael Gromov and H. Blaine Lawson, Jr. The classification of simply connected manifolds of positive scalar curvature. Ann. of Math. (2), 111(3):423-434, 1980.

[GL83] Mikhael Gromov and H. Blaine Lawson, Jr. Positive scalar curvature and the Dirac operator on complete Riemannian manifolds. Inst. Hautes Études Sci. Publ. Math., 58:83-196 (1984), 1983.

[GMW] Hao Guo, Varghese Mathai, and Hang Wang. Positive scalar curvature and poincare duality for proper actions. https:// arxiv.org/abs/1609.01404.

[Gro96] M. Gromov. Positive curvature, macroscopic dimension, spectral gaps and higher signatures. In Functional analysis on the eve of the 21st century, Vol. II (New Brunswick, NJ, 1993), volume 132 of Progr. Math., pages 1-213. Birkhäuser Boston, Boston, MA, 1996. 
[Hat] Allen Hatcher. Notes on basic 3-manifold topology. https:// www . math. cornell.edu/ hatcher/.

[Hil02] J. A. Hillman. Four-manifolds, geometries and knots, volume 5 of Geometry \& Topology Monographs. Geometry \& Topology Publications, Coventry, 2002.

[HK01] Nigel Higson and Gennadi Kasparov. E-theory and $K K$-theory for groups which act properly and isometrically on Hilbert space. Invent. Math., 144(1):23-74, 2001.

[HPS15] Bernhard Hanke, Daniel Pape, and Thomas Schick. Codimension two index obstructions to positive scalar curvature. Ann. Inst. Fourier (Grenoble), 65(6):2681-2710, 2015.

[HR00] Nigel Higson and John Roe. Analytic K-homology. Oxford Mathematical Monographs. Oxford University Press, Oxford, 2000. Oxford Science Publications.

[Ill00] Sören Illman. Existence and uniqueness of equivariant triangulations of smooth proper $G$-manifolds with some applications to equivariant Whitehead torsion. J. Reine Angew. Math., 524:129$183,2000$.

[Kas88] G. G. Kasparov. Equivariant $K K$-theory and the Novikov conjecture. Invent. Math., 91(1):147-201, 1988.

[Koc96] S. O. Kochman. Bordism, stable homotopy and Adams spectral sequences, volume 7 of Fields Institute Monographs. American Mathematical Society, Providence, RI, 1996.

[KW75a] Jerry L. Kazdan and F. W. Warner. Existence and conformal deformation of metrics with prescribed Gaussian and scalar curvatures. Ann. of Math. (2), 101:317-331, 1975.

[KW75b] Jerry L. Kazdan and F. W. Warner. Scalar curvature and conformal deformation of Riemannian structure. J. Differential Geometry, 10:113-134, 1975.

[Las63] R. Lashof. Poincaré duality and cobordism. Trans. Amer. Math. Soc., 109:257-277, 1963.

[LM89] H. Blaine Lawson, Jr. and Marie-Louise Michelsohn. Spin geometry, volume 38 of Princeton Mathematical Series. Princeton University Press, Princeton, NJ, 1989.

[Loh] J. Lohkamp. The higher dimensional positive mass theorem ii. https://arxiv.org/abs/1612.07505. 
[Lüc02a] Wolfgang Lück. Chern characters for proper equivariant homology theories and applications to $K$ - and $L$-theory. J. Reine Angew. Math., 543:193-234, 2002.

[Lüc02b] Wolfgang Lück. $L^{2}$-invariants: theory and applications to geometry and $K$-theory, volume 44 of Ergebnisse der Mathematik und ihrer Grenzgebiete. 3. Folge. A Series of Modern Surveys in Mathematics [Results in Mathematics and Related Areas. 3rd Series. A Series of Modern Surveys in Mathematics]. SpringerVerlag, Berlin, 2002.

[Mil56] John Milnor. Construction of universal bundles. I. Ann. of Math. (2), 63:272-284, 1956.

[Mil63] J. Milnor. Spin structures on manifolds. Enseignement Math. (2), 9:198-203, 1963.

[NSZ] Martin Nitsche, Thomas Schick, and Rudolf Zeidler. Notes on obstructions to psc via submanifolds. in preparation.

[Ran02] Andrew Ranicki. Algebraic and geometric surgery. Oxford Mathematical Monographs. The Clarendon Press, Oxford University Press, Oxford, 2002. Oxford Science Publications.

[Ros86] J. Rosenberg. $C^{*}$-algebras, positive scalar curvature and the Novikov conjecture. II. In Geometric methods in operator algebras (Kyoto, 1983), volume 123 of Pitman Res. Notes Math. Ser., pages 341-374. Longman Sci. Tech., Harlow, 1986.

[RS01] Jonathan Rosenberg and Stephan Stolz. Metrics of positive scalar curvature and connections with surgery. In Surveys on surgery theory, Vol. 2, volume 149 of Ann. of Math. Stud., pages 353-386. Princeton Univ. Press, Princeton, NJ, 2001.

[Sch98] Thomas Schick. A counterexample to the (unstable) GromovLawson-Rosenberg conjecture. Topology, 37(6):1165-1168, 1998.

[Smi78] Justin R. Smith. Complements of codimension-two submanifolds. The fundamental group. Illinois J. Math., 22(2):232-239, 1978.

[Sto92] Stephan Stolz. Simply connected manifolds of positive scalar curvature. Ann. of Math. (2), 136(3):511-540, 1992.

[Sto02] Stephan Stolz. Manifolds of positive scalar curvature. In Topology of high-dimensional manifolds, No. 1, 2 (Trieste, 2001), volume 9 of ICTP Lect. Notes, pages 661-709. Abdus Salam Int. Cent. Theoret. Phys., Trieste, 2002. 
[SY79] R. Schoen and S. T. Yau. On the structure of manifolds with positive scalar curvature. Manuscripta Math., 28(1-3):159-183, 1979.

[SY17] R. Schoen and S. T. Yau. Positive scalar curvature and minimal hypersurface singularities. preprint, https://arxiv.org/abs/ 1704.05490, 2017.

[Val02] Alain Valette. Introduction to the Baum-Connes conjecture. Lectures in Mathematics ETH Zürich. Birkhäuser Verlag, Basel, 2002. From notes taken by Indira Chatterji, With an appendix by Guido Mislin.

[Wie16] Michael Wiemeler. Circle actions and scalar curvature. Trans. Amer. Math. Soc., 368(4):2939-2966, 2016.

[Zei17] Rudolf Zeidler. An index obstruction to positive scalar curvature on fiber bundles over aspherical manifolds. to appear in Algebr. Geom. Topol., https://arxiv.org/abs/1512.06781, 2017. 